\title{
A Novel Microbial Source Tracking DNA Microarray Used for Pathogen Detection in Environmental Systems
}

Xiang Li

Follow this and additional works at: https://researchrepository.wvu.edu/etd

\section{Recommended Citation}

Li, Xiang, "A Novel Microbial Source Tracking DNA Microarray Used for Pathogen Detection in Environmental Systems" (2015). Graduate Theses, Dissertations, and Problem Reports. 6072. https://researchrepository.wvu.edu/etd/6072

This Dissertation is protected by copyright and/or related rights. It has been brought to you by the The Research Repository @ WVU with permission from the rights-holder(s). You are free to use this Dissertation in any way that is permitted by the copyright and related rights legislation that applies to your use. For other uses you must obtain permission from the rights-holder(s) directly, unless additional rights are indicated by a Creative Commons license in the record and/ or on the work itself. This Dissertation has been accepted for inclusion in WVU Graduate Theses, Dissertations, and Problem Reports collection by an authorized administrator of The Research Repository @ WVU.

For more information, please contact researchrepository@mail.wvu.edu. 


\title{
In Vitro Recapitulation of Functional Microvessel Using Microfluidic Platform
}

\author{
by \\ Xiang Li \\ Dissertation submitted to the \\ at West Virginia University \\ in partial fulfillment of the requirements \\ for the degree of \\ Doctor of Philosophy \\ in \\ Electrical Engineering \\ Yuxin Liu, Ph.D., Chairperson \\ Thirimachos Bourlai, Ph.D. \\ Jeremy Dawson, Ph.D. \\ Pingnian He, Ph.D. \\ Larry A. Hornak, Ph.D. \\ Nianqiang Wu, Ph.D.
}

Benjamin M. Statler College of Engineering and Mineral Resources

Lane Department of Computer Science and Electrical Engineering

Morgantown, West Virginia

November, 2014

Keywords: microfluidics, microvessel model, primary endothelial cells, barrier functions 


\section{ABSTRACT \\ In Vitro Recapitulation of Functional}

\section{Microvessel Using Microfluidic Platform}

\section{Xiang Li}

Microfluidic technologies have enabled in vitro studies to mimic in vivo microvessel environment with sufficient complexity. However, there are still existing knowledge gaps and lack of convincing evidence to demonstrate and quantify key features of a functional microvessel. In this dissertation, a physiologically realistic microvessel model was developed with a stable and mature endothelium for studying complex vascular phenomena, such as endothelial cell signaling and barrier functions with the microscopic resolution at individual cellular levels. With advanced micromanufacturing and microfluidic technologies, two types of cost-efficient, easy to operate and reproducible microchannel network devices were fabricated, and the fabricated microchannels mimicked the dimension of in vivo microvessels. With long-term and continuous perfusion control, seeded endothelial cells were able to maintain their phenotype, viability, proliferation with proper barrier functions, and respond to flow shear force and inflammatory stimuli. In particular, primary human umbilical vein endothelial cells were successfully cultured the entire inner surface of the microchannel network with well-developed VE-cadherin junctions throughout the channels. The endothelial cell response to shear stresses were quantified under different shear stress conditions and demonstrated their morphological changes close to those reported in venular vasculature. Furthermore, real time agonist-induced changes in intracellular $\mathrm{Ca}^{2+}$ concentration $\left[\mathrm{Ca}^{2+}\right]_{i}$ and nitric oxide $(\mathrm{NO})$ production was successfully measured by integrating microvessel model into microscopic systems. The results were similar and comparable to those derived from individually perfused intact venules. With the validation of its functionalities, this microfluidic model demonstrates a great potential for biological applications and bridging the gaps between in vitro and in vivo microvascular research. 


\section{Acknowledgment}

First and foremost I would like to thank my advisor and mentor Dr. Yuxin Liu, for all her guidance, inspiration, and encouragement. It is my extreme privilege to have been the graduate research assistant of her lab for the past four years. I am extremely lucky to be given the chance joining her group, and continuously improving my research ability in the field of microfluidics. She is not only my advisor of the research work, but also my mentor of life. The past four years have been a big turning point for me and my family, and without all her helps and supports, there was no way I could achieve what I have done today.

I would like to state my gratitude to Dr. Pingnian He as the collaborator of the microvessel model project. Without her fully support and extremely valuable guidance, this project would not become one of the most elegant and well-validated studies in this field.

I gratefully acknowledge the members of my committee: Dr. Thirimachos Bourlai, Dr. Jeremy Dawson, Dr. Pingnian He, Dr. Larry A. Hornak, and Dr. Nianqiang Wu, for their valuable time and guidance of my research work as well as the valuable feedback on this dissertation.

I am very thankful to my lab member: Jianbo Sun and Veronica Betancur Calle, and all my former lab members: Zhouchun Huang, Samantha Mearns, Bhargavi Thotakura, Hanyuan Zhang and Ruizhi Zhong. I would like to thank Dr. Sulei Xu from Dr. He's group, for all her devotion and help on this research project.

I have saved the last word of acknowledgement for my extended family for all their love, support, and sacrifices. Without them, this dissertation would never have been written. 


\section{Table of Contents}

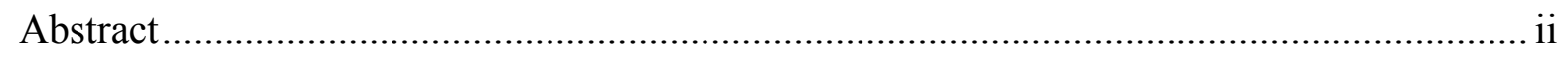

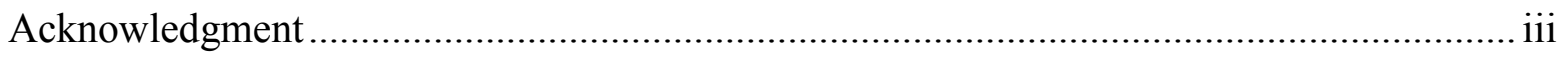

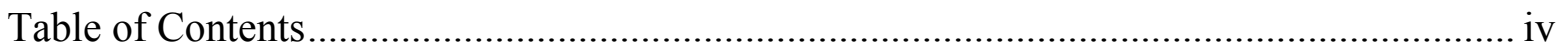

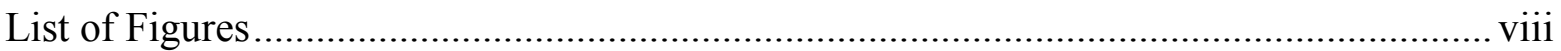

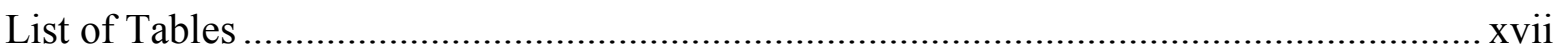

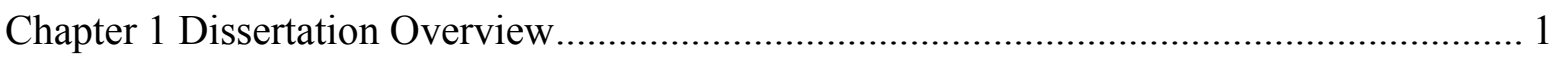

1.1 Background and motivations ....................................................................... 1

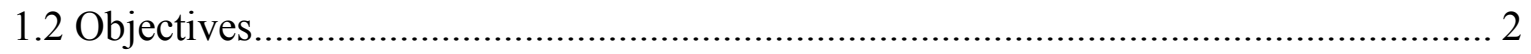

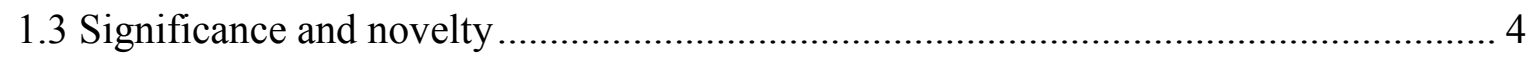

1.4 Structure of the dissertation ............................................................................. 5

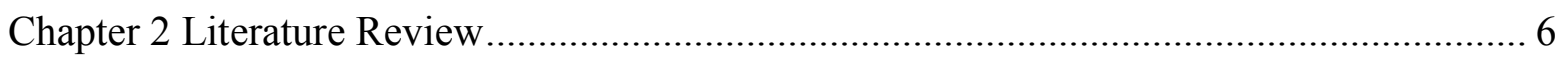

2.1 Conventional microvessel models ............................................................... 6

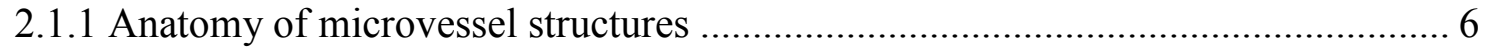

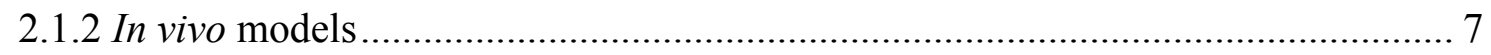

2.1.3 Conventional in vitro models................................................................... 10

2.2 Microfluidic model for microvascular research .............................................. 15

2.2.1 Materials used for microfluidic device fabrication...................................... 16

2.2.2 Microvascular cell culture and testing .................................................... 16 


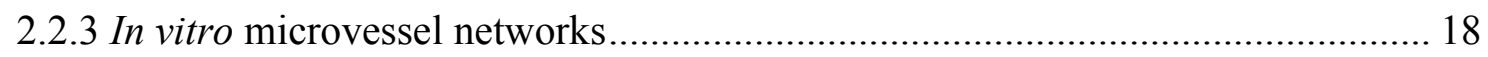

2.2.4 The application of microfluidic microvascular model...................................... 20

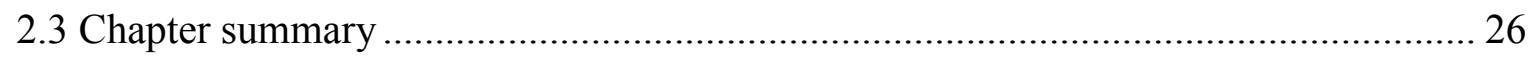

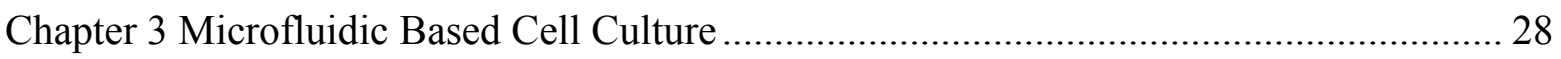

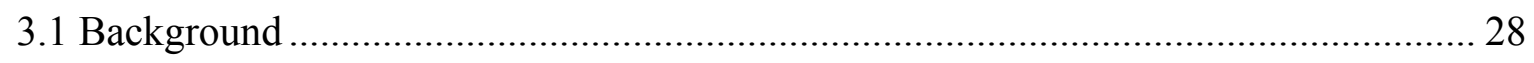

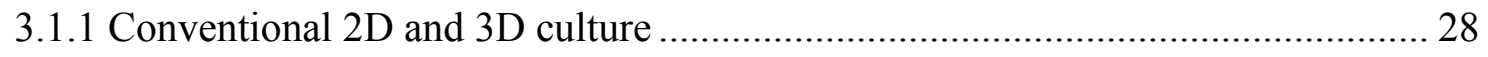

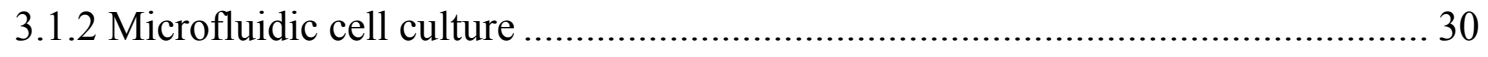

3.2 Long term perfusion system developed for microvessel model .................................. 34

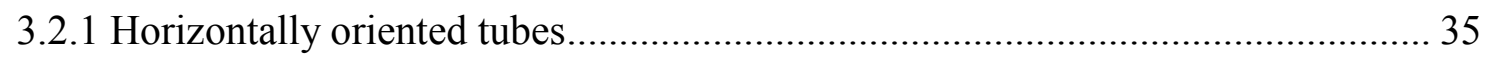

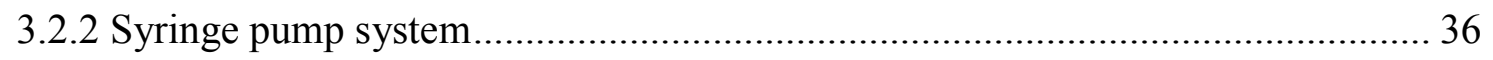

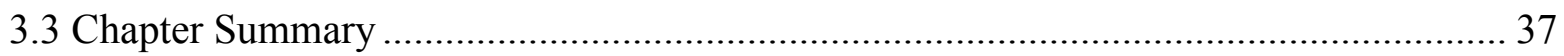

Chapter 4 Standard Fabrication Method for Microfluidic Microchannel Networks .............. 39

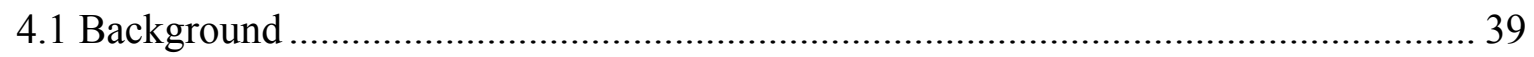

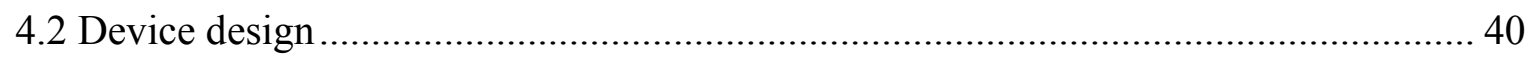

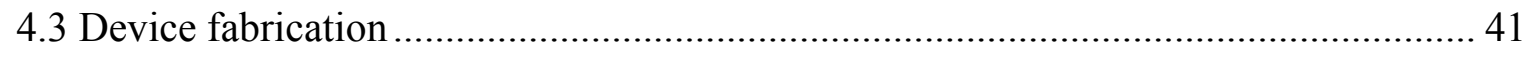

4.4 Endothelial cell seeding and long-term perfusion....................................................... 43

4.4.1 Endothelial cells seeding and culture method ..................................................... 43

4.4.2 Long-term cell culture under constant perfusion................................................. 45

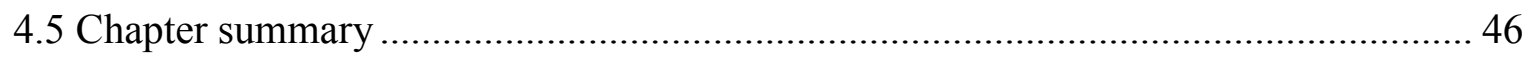

Chapter 5 Multi-Diameter Microfluidic Microchannels........................................................ 47 


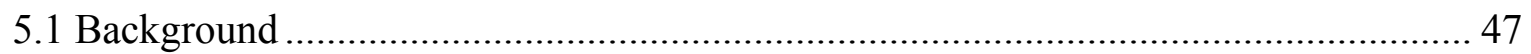

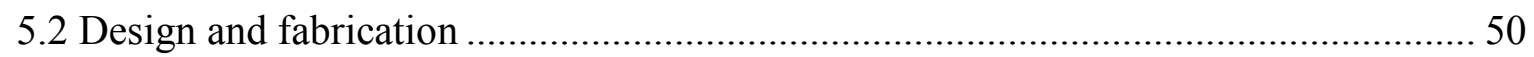

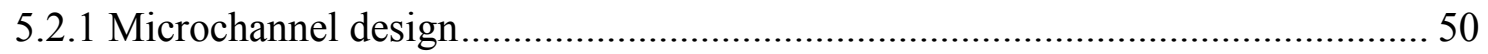

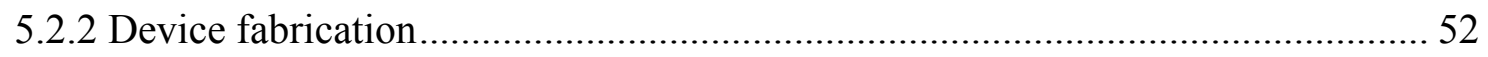

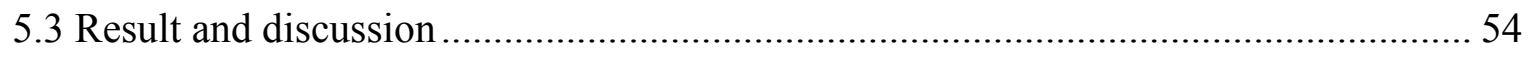

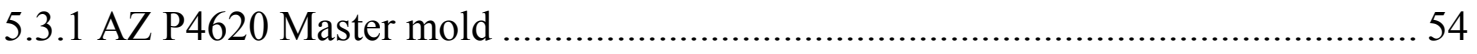

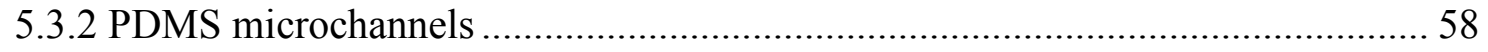

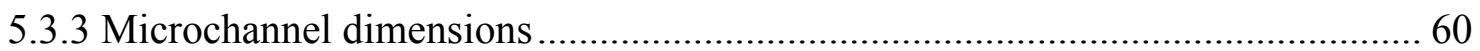

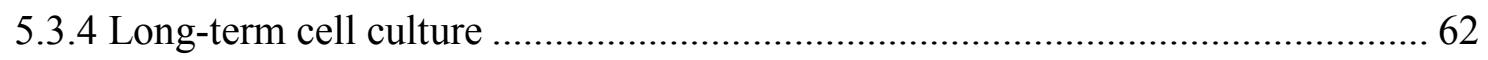

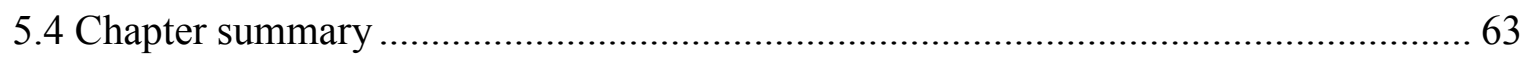

Chapter 6 Characterization of Endothelial Junctions and Cell Morphology Analysis in

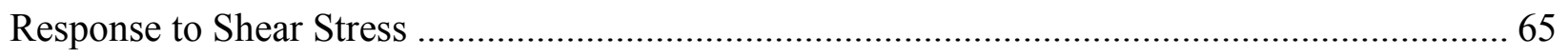

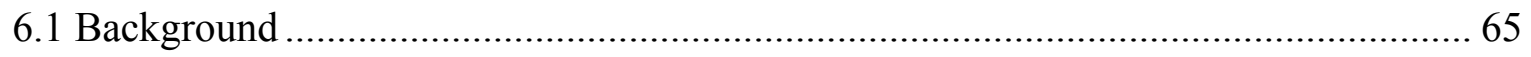

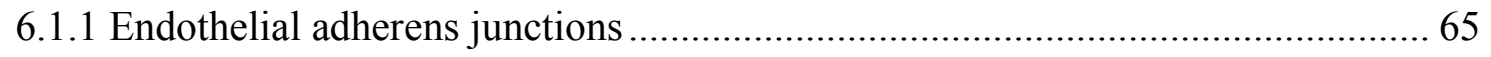

6.1.2 Cell morphology analysis in response to shear stress.......................................... 69

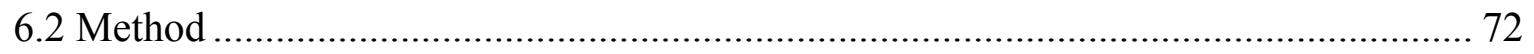

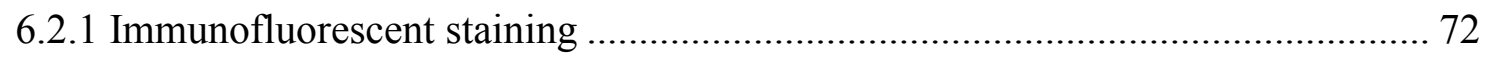

6.2.2 Analysis of cell morphology in response to shear stress .................................... 73

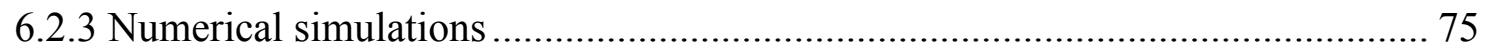

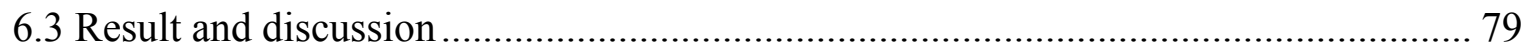


6.3.2 Cell morphology analysis in response to shear stress....................................... 80

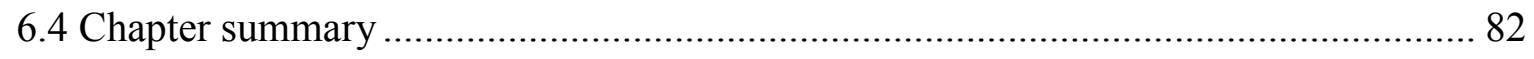

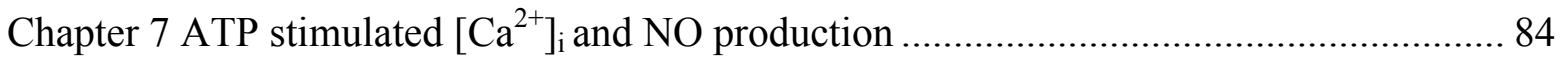

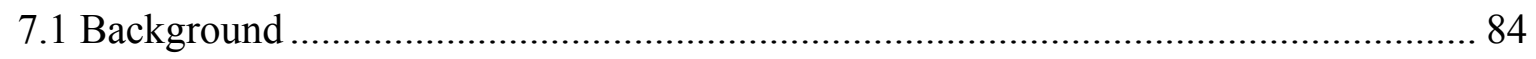

7.1.1 $\left[\mathrm{Ca}^{2+}\right]_{\mathrm{i}}$ and NO as key parameters to represent functional microvessel ............... 85

7.1.2 Endothelial $\left[\mathrm{Ca}^{2+}\right]_{\mathrm{i}}$ and NO stimulated by ATP .............................................. 89

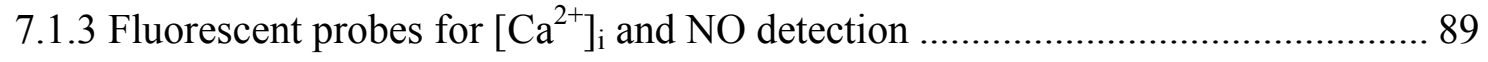

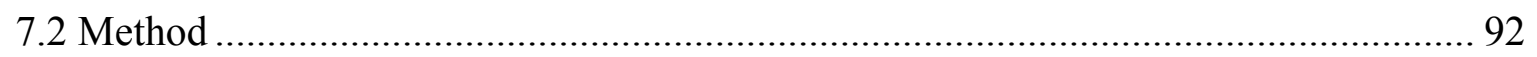

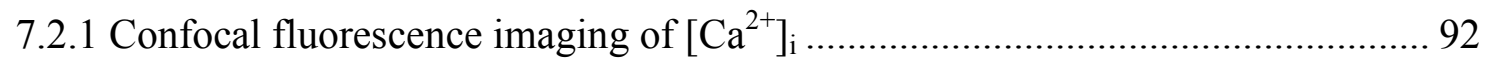

7.2.2 Fluorescence imaging of NO production............................................................. 94

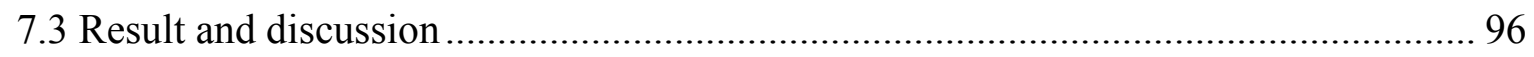

7.3.1 Endothelial $\left[\mathrm{Ca}^{2+}\right]_{\mathrm{i}}$ change induced by ATP .................................................. 96

7.3.3 Measurement of ATP induced NO production ................................................... 98

7.3.3 Discussion of ATP induced $\left[\mathrm{Ca}^{2+}\right]_{\mathrm{i}}$ change and NO production ....................... 100

Chapter 8 Conclusions and Future Work....................................................................... 103

8.1 Summary of major contributions ........................................................................ 103

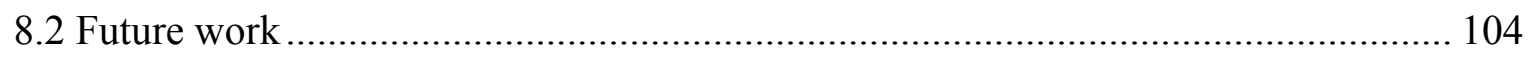

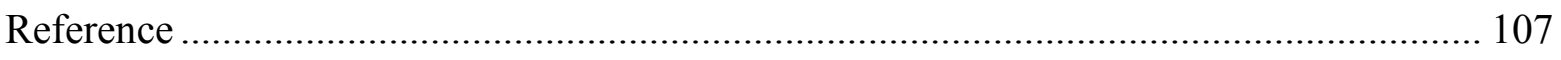




\section{List of Figures}

Figure 2.1 a) Microvascular architecture in skeletal muscle. Capillaries (indicated as $\mathrm{Ca}$ ) are oriented longitudinally in the same direction as the muscle fibers they supply. The capillary network is branching out from an arteriole (indicated as Ar) and joining back together to form a venule (indicated as $\mathrm{Ve}$ ). b) Electron micrograph of a cross section of a small capillary in the pancreas. The wall is formed by a single endothelial cell surrounded by a basal lamina. Note the small transcytotic vesicles, which according to one theory provide transport of large molecules in and out of this type of capillary: materials are taken up into the vesicles by endocytosis at the luminal surface of the cell and discharged by exocytosis at the external surface, or vice versa.... 7 Figure 2.2 a) A dorsal skinfold window chamber model on mice and the enlarged white light microscopy of entire window. b) A typical rat mesenteric window assay. c) Miles assay on the ear of untreated and 3TSR-treated mice. d) CAM assay is a low-cost method for assessing angiogenic factors in an established vessel system. e) The tale of zebra fish show the ability to discern structures on the cellular level.

Figure 2.3 a) Endothelial permeability assay performed on transwell system shows VEGFinduced loosening of cell-cell junctions. b) Scheme of matrigel invasion assay of endometrial stromal cells (ESCs) or AC-1M88 a model for extravillous trophoblast cells. c) Characterization of polarized epithelial cells by measuring transepithelial resistance with a voltohmmeter d) Schematic diagram of cell culture platforms to mimic the liver sinusoid.

Figure 2.4 a) The cells cultured in the center of the plate did not show any alignment with orbital shaker. b) A cone-and-plate system complying with the dimensional requirements to limit secondary flow effects. c) An illustration of the flow chamber. d) The testing set up using a commercialized flow chamber. e) The height variation of the flow chamber (showed in cross- 
section view) would result in a wall shear tress (WSS) variation. (f) Schematic diagram of flow chamber design for a variable shear stress Hele-Shaw device. ………………………............... 14

Figure 2.5 a) Parallel microchannels with syringe injectors to provide a controlled flow rate. b) Short-term adhesion multisample device accommodating identical channels. c) Schematic of three-chamber rmicrofluidic device. Top view shows positions of reagent channels and cell culture chamber. Microcapillaries with the width of $5 \mu \mathrm{m}$ connect the reagent channels to the cell culture chamber. On the cross-section view, the thickness of each layer is marked where the dashed lines show positions of the inlets and outlets at both ends. d) Schematic for microfluidic cell migration assay base on multi-chamber channel with a hydrogel region. In this set-up, it is possible to compare the cell migration behavior between the condition and control sides directly.

Figure 2.6 a) Use of perfused microfluidic gels as scaffolds for cell culture. High magnification image shows collagen gel with a monolayer of endothelial cells lining. Scale bars: $200 \mu \mathrm{m}$. b) A three-dimensional endothelial-lined lumens were patterned in collagen filled microchannels using basic fluid principles such as passive pumping and viscous fingering of fluids with different viscosities. Scale bars: $500 \mu \mathrm{m}$. c) Engineered 3D microvessels characterized by immunofluorescence: confocal micrographs showing the overall architectures of vascular networks established by vasculogenesis. Scale bars: $100 \mu \mathrm{m}$. d) Confocal sections of endothelialized microfluidic vessels of corner and branching sections. Red, CD31; blue, nuclei. Scale bar: $100 \mu \mathrm{m}$. e) 3D renderings of multiple confocal microscopy using fluorescent cell membrane (red) and cell nuclear (blue) dyes show that the endothelial cells line the entire inner surface of the microfluidic channels. Scale bars: $30 \mu \mathrm{m}$. 
Figure 2.7 The passage of cells and macromolecules through the endothelial barrier can occur through transcellular (vesicular vacuolar organelles) or paracellular (tight and adherens junctions) pathways. Gap junctions ensure water and ion transport. Moreover, endothelial cells are anchored and connected to the extracellular matrix (ECM) through integrin-based adhesion complexes, namely focal adhesions.

Figure 3.1 Cytoskeleton reorientation of endothelial cell under shear stress. a) HUVECs were cultured under static condition. When reaching confluence, the cells were fixated with paraformaldehyde and stained for actin filaments (green). b) After subjected cell to shear stress, most filaments were aligned and oriented in the flow direction that was indicated by the arrow. Scale bar: $50 \mu \mathrm{m}$. 29

Figure 3.2 Illustration of small-volume effects in microfluidic cell culture devices. Smaller culture media volume results in faster consumption of nutrients and increased concentration of metabolites or secreted molecules, similar to tightly packed tissues. 32 Figure 3.3 a) The hydraulic pressure caused by height difference between the horizontally oriented tubes becomes the driven force of the perfusion flow. b) The actual image of the

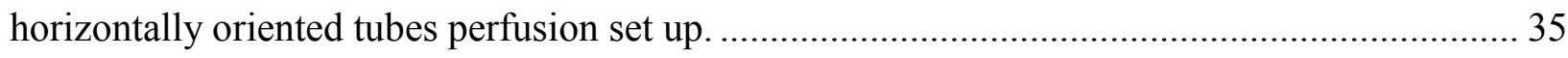

Figure 3.4 Average flow speed $(\mathrm{mm} / \mathrm{s})$ measured in the smallest daughter channel.................... 36 Figure 3.5 Schematic diagram showing the long-term perfusion through the microvessel model

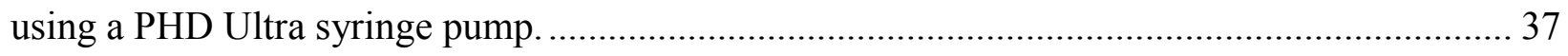

Figure 4.1 The schematic designs of the microchannel networks from the CAD file.................. 40 Figure 4.2 The schematic fabrication procedures for PDMS devices. a-b) SU-8 photoresist was spin-coated onto the pre-cleaned silicon wafer. c) The photoresist was exposed to UV light though the photomask. d) Developed flow chamber/microchannel pattern as the master mold. e) 
PDMS solution was casted onto the master mold and cured. f) The flow chamber/PDMS microchannel structure was bonded onto the substrate.

Figure 4.3 a) Bright field image of the HUVEC initial loading. b) Bright field image of the HUVEC attachment $15 \mathrm{~min}$ after the initial loading. Scale bars: $100 \mu \mathrm{m}$. 44

Figure 4.3 The representative confocal images show the HUVECs successfully cultured throughout the inner surfaces of the entire microchannel network. a) The schematic image of the network with selected regions as shown in b-d. b-d) HUVECs were stained with F-actin and cell nuclei in each region, where b1, $\mathrm{c} 1$ and $\mathrm{d} 1$ show the top surfaces of the channel, respectively. $\mathrm{b} 2$, c2 and d2 show the bottom surfaces of the channel, respectively. e) The cross-sectional images at each region. The locations of the cross-sections were indicated as 1-1', 2-2', and 3-3' in b1-d1, respectively. Scale bars: $100 \mu \mathrm{m}$. 45

Figure 5.1 Schematic designs of a single microchannel and a microchannel network. a) A straight channel. (Width: $100 \mu \mathrm{m}$ and length: $8 \mathrm{~mm}$ as an example) b) A network with multi-branch channels. As an example, the channel widths at different branch channels were calculated based on the Murray's law. 51

Figure 5.2 The schematic fabrication procedures for AZ P4620 master molds. a) A precleaned silicon substrate. b) Two layers of positive photoresist AZ P4620 were spun onto the silicon substrate, respectively. The second layer of AZ P4620 was applied after the first layer was baked and dried. c) The photoresist was exposed under the UV light through the mask. d) The patterned structure after AZ $400 \mathrm{~K}$ development. e) The reflow photoresist patterns with semi-circular cross-sections after the reflow at $120^{\circ} \mathrm{C}$ for 4 minutes 53

Figure 5.3 The schematic fabrication procedures for cylindrical microchannels in PDMS. a) The reflow AZ P4620 microchannel network. b) Cast PDMS solution onto the AZ P4620 mold. c) 
The cured PDMS layer. d) Aligned and bonded two PDMS layers to form microchannels with circular cross-sections. 54

Figure 5.4 SEM shows the developed AZ P4620 photoresist. a) before reflow b) after reflow. c) Molded PDMS showed the cross section of the resist before reflowing; the picture shows a rectangular cross section. d) Molded PDMS showing a semi-circular cross-section of the resist after reflowing. The cross section after reflow was controlled by the initial dimension design of the pattern and reflow temperature. 55

Figure 5.5 SEM shows small defects on the reflowed mold which is caused by high reflow baking temperatures $\left(>120^{\circ} \mathrm{C}\right)$ or long baking time ( $>4$ minutes). 57

Figure 5.6 Microscope images show the smooth surface transitions at bifurcations. a) A PDMS channel network. The frames indicate the bifurcations through the channel network and the arrows indicate the panels at the right side. The scale bar is $500 \mu \mathrm{m}$. b-e) Optical images show the smooth surface transitions on the PDMS channel network. The scale bar is $50 \mu \mathrm{m}$. $\mathrm{f}-\mathrm{i}$ ) SEM images show the smooth surface transitions on the AZ P4620 resist mold. The scale bar is $50 \mu \mathrm{m}$.

Figure 5.7 a) An aligned and bonded cylindrical microchannel network in PDMS. b-d) Circular cross-sections of PDMS molds show channel dimensions at each level (1-1', 2-2', and 3-3').... 60 Figure 5.8 Microscopy images using fluorescent cell membrane dye (red) and cell nuclear dye (blue) show that the HUVECs line the inner surface of a cylindrical microchannel network at difference branching regions. a) Top, b) Middle, and c) Bottom. d) Confocal microscopy image shows the circular cross-sectional view of HUVECs lining around the channel network. Scale bars: $100 \mu \mathrm{m}$. 63 
Figure 6.1 Endothelial cell junctions and adhesions. Endothelial cells of the microvessel wall are joined together by intercellular junction proteins: adherens junction, tight junction and/or gap junction. Barrier function in most vascular beds is provided by adherens junction. Only some specific microvasculatures rely on tight junction for additional barrier function, and gap junction does not directly contribute to barrier functions, but facilitate signaling between cells. 66 Figure 6.2 Molecular structures of endothelial adherens junctions. VE-cadherin is indicated as a dimer that is the minimal functional unit of cadherins. Five homologous extracellular domains are shown as EC1- EC5. At cell-to-cell contacts, the cluster of VE-caherin proteins is important for certain signaling/regulating mechanism and performs as a scaffold for other complex protein clusters. The proteins that are known to interact with VE-cadherin include the catenin proteins p120, $\beta$ cat and plako. $\beta$ cat and plako is directly associated with VE-cadherin and $\alpha$-cat. 67

Figure 6.3 a) The VE-cadherin staining of HUVEC maintained in static culture condition. b) After exposed to shear stress at $10 \mathrm{dyne} / \mathrm{cm}^{2}$, the intensity of VE-cadherin staining was increased, and the intricate latticework appeared to broaden. c) By 47 hours, the VE-cadherin was reorganized into compact junctions at the endothelial cell interfaces. 1 and 2 are the $2 \times$ magnifications of the boxed areas indicated in a and c. Scale bar: $25 \mu \mathrm{m}$. d) VE-cadherin staining of intact venule from animal model.

Figure 6.3 Structures of actin and diagrams of fundamental reactions. a) Ribbon and space-filling models of the actin molecule (pdb:1ATN). b) Spontaneous nucleation and elongation. Longer polymers grow rapidly at the barbed end (B) and slowly at the pointed end (P). c) Actin monomer binding various proteins. d) Nucleation and elongation by formins. e) Nucleation by Arp2/3 complex. f) Reactions of actin filaments. Capping proteins bind to and block barbed ends; cofilin and gelsolin sever filaments; cross-linking proteins assemble networks and bundles of 
actin filaments. g) Myosin motors, such as myosin V, use cycles of ATP hydrolysis to walk along actin filaments. 70

Figure 6.4 a) The breakdown and tearing of VE-Cadherin after fixing, as VE-Cadherin shows dashed line connecting cells. b) After probably adjust the concentration of fixing media and loading speed, the VE-Cadherin was distributed normally. Scale bar: $50 \mu \mathrm{m}$. 73

Figure 6.5 Comparing different permeabilizing protocols of the F-actin staining. a) When the cells were treated with Triton for 10 minutes, there was a significant level of nonspecific background staining. b) After 5 minutes of Triton treatment, image quality of the microscopy was greatly improved. The images show the same branching region from two different devices with different permeabilizing duration. Scale bar: $50 \mu \mathrm{m}$. 74

Figure 6.6 The numerical 3D model built with CFD commercial software COMSOL. a) Wall shear stress distribution of the whole networks, where the selected regions of $\mathrm{b}$ and $\mathrm{c}$ were indicated. Scale bars: $500 \mu \mathrm{m}$. b-c) The cross-section view of the shear stress at different branching section. Scale bars: $100 \mu \mathrm{m}$. 78

Figure 6.7 The representative immunofluorescent confocal images demonstrated the VECadherin junctions formation throughout the entire network. a) The schematic image of the network with selected regions as shown in b-d. b-c) VE-Cadherin and cell nuclei staining were shown at the first and third branching regions. $\mathrm{d}_{1}-\mathrm{d}_{2}$ ) VE-Cadherin and cell nuclei staining were shown at the top and bottom surfaces of the second branching regions, respectively. e) Enlarged

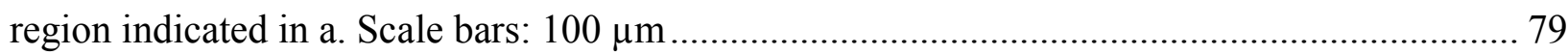
Figure 6.8 HUVECs response to the different levels of shear stresses: The morphology change was studied with F-actin staining. a) The schematic image of the network indicated with selected region for shear stress test. b) COMSOL simulation showed the wall shear stress distribution in 
the selected region along the network. c-e) Confocal image showed the F-actin stress fibers under the LSC-LST, LSC-HST, and HSC-HST respectively. f) The aspect ratio was compared between the three testing groups. g) Comparing of the cell surface areas between the testing groups. All data are reported as the means \pm SE of independent experiment. Scale bars: $100 \mu \mathrm{m}$.

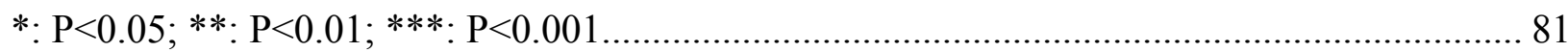

Figure 7.1 The regulation function of Cav-1 and CaM on eNOS in endothelium. a) eNOS that bound to caveolin-1 (Cav-1) on the inner leaflet of the plasma membrane is inactive. b) Dimerized eNOS becomes activated by $\mathrm{Ca}^{2+} / \mathrm{CaM}$ that is bound to the same site as Cav-1. eNOS

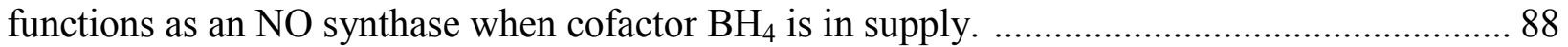

Figure 7.2 a) The chemical structures of fluo-3 and fluo-4. b) Double label imaging shows that fluo-4-labeled cells were brighter than fluo-3-labeled cells. The green label from double-labeled cells (fluo-3-filled with red halo) was much dimmer than the green label in fluo-4-filled cells. 90

Figure 7.3 Schemes for the fluorescence signal activation of fluorescein-based probes reacted with NO. a) Key reaction of benzotriazole formation. b) DAF-2 is converted to a triazole compound DAF-2 $\mathrm{T}$ and cause slightly change of maximum absorbance but significant change in fluorescence intensity. 91

Figure 7.4 The schematic procedures for endothelial $\left[\mathrm{Ca}^{2+}\right]_{i}$ detection induced by ATP. a) After endothelial cells reached confluence, the device was perfused with albumin-Ringer solution. b) Fluo-4 was perfused into the device for 40 minutes within the incubator. c) The device was first perfused with albumin-Ringer for baseline measurement, then $10 \mu \mathrm{M}$ ATP to detect the $\left[\mathrm{Ca}^{2+}\right]_{\mathrm{i}}$ response. d) The confocal image was stacked with maximum intensity projection, and each area 
of individual endothelial cells was manually selected as ROI, and processed by Metafluor software. 93

Figure 7.5 The schematic procedures for NO detection. a) Device was rinsed with albuminRinger. b) DAF-2 DA was perfused for 40 minutes within the incubator. c) The basal NO production and FI change were measured with the microscopic system. d) After manually select each ROI, the image data was processed to calculate the changes in $\mathrm{FI}_{\mathrm{DAF}}$ 96 Figure 8.1 a) A microfluidic microchannel design to study the released ATP from erythrocytes under shear flow. The confined region was designed to apply sudden change of shear stress on the perfused red blood cells. b) The shear rate distribution under the flow of $0.3 \mu \mathrm{L} / \mathrm{min}$. c) a microvessel model was designed to mimic a clotting microvessel. d) The corresponding shear rate under the flow of $0.3 \mu \mathrm{L} / \mathrm{min}$. 106 


\section{List of Tables}

Table 3.1 Basic requirements for cell culture, and improvements when microfluidic methods are

used.

Table 3.2 Required characteristics of pumps for cell culture and manipulation. 34

Table 5.1 The measurement of microchannel cross-sectional dimension. (unit: $\mu \mathrm{m}$ ) 62

Table 6.1 Summary of flow rate test........ 74 


\section{Chapter 1 Dissertation Overview}

\subsection{Background and motivations}

Microvasculature, which is the network of microvessels, works as a part of the circulation system and extended organ, mediates the interactions between blood and tissues, supports metabolic activities, defines tissue microenvironment, and plays a critical role in many health and pathological conditions. The vascular endothelium (a monolayer of endothelial cells) lining the inner surface of blood vessels serves as the first interface for circulating blood components to interact with cells of the vascular wall and surrounding extravascular tissues. It is a major player in the control of blood fluidity, platelet aggregation and vascular tone, a major actor in the regulation of immunology, inflammation and angiogenesis, and an important metabolizing and an endocrine organ. (Félétou 2011) Blood vessels related pathologies are present in many medical conditions. In vitro recapitulation of functional microvessels to mimic a more pathological/physiological environment is critically needed for comprehensive and quantitative studies of blood-endothelium interactions and complex vascular phenomena, and for better understanding vascular pathology to design effective therapeutic strategies.

In exchange for experimental convenience, many of current in vitro models sacrifice physiological accuracy. Vascular cells are normally cultured on planar plates, filters, or these substrates coated with hydrogels (Lal, Varma et al. 2001; Breslin, Pappas et al. 2003; Chen, Somanath et al. 2005; Nobe, Sone et al. 2005; Chandra, Barillas et al. 2007). In those models, a microvasculature is randomly generated by cellular self-assembly. These systems have inherent difficulties in achieving a controlled flow over vascular cells, or attaining interactions between the blood-endothelium. The most common way to replicate certain features of in vivo shear stress by biologist includes parallel flow chamber and cone and plate viscometer.(Chiu and Chien 2011) 
These two models lack the ability to apply a wide range of uniform and stable shear stresses. Furthermore, complex geometries of in vivo microvasculature and miniaturization for real-time monitoring of small vessels are difficult to obtain. Although animal models have vastly improved our understanding of vascular biology and pathophysiology, they are very expensive, highly variable, difficulty to manipulate, and experimental results may be confounded.

Microfluidics has the advantage to provide a solution that has not been addressed by those macroscale approaches, such as developing a more physiologically relevant in vitro model. However, the adaptation and application of microfluidics in mainstream biology is still lacking. According to the recent summary, the majority publications of microfluidics are still in engineering journals (85\%). (Sackmann, Fulton et al. 2014) The equivalent or improved performance of microfluidic devices is not convincing enough for many biologists to switch from current state-of-the-art techniques such as transwell assay and macroscale culture dish/glass slide. (Berthier, Young et al. 2012; Sackmann, Fulton et al. 2014)

\subsection{Objectives}

The main objective of this dissertation is to develop a physiologically realistic functional microvessel model that mimic the geometries and fluidic environment of in vivo microvessels, recapitulate key features of the microvessels in vivo, and validated its utility for biological applications. The developed microvessel model is expected to overcome critical constraints of current in vitro models, in which a stable and mature endothelium is normally lack as well as appropriate barrier functions. With the successfully development of the model, it could provide a platform for the study of complex vascular phenomena. Specifically, this dissertation focuses on: 
1) Create a microchannel network mimicking geometries of in vivo microvessels: Two different fabrication methods were presented. One method was to fabricate a multi-diameter circular microchannel network by photoresist reflowing technique and polydimethylsiloxane (PDMS) replication. The second method used the standard SU-8 photolithography and PDMS soft lithography and the resulted microchannel network can easily incorporate confocal microscopes for imaging.

2) Develop strategies to set and optimize shear flow conditions through the channel network. To maintain a steady laminar flow within the microchannel networks, two different long term perfusion methods were developed: the horizontally oriented tubes and syringe pump system. The optimized perfusion is expected to be performed up to two weeks. Furthermore, computational fluid dynamic (CFD) models were used to simulate the flow profile and corresponding distributions of shear stresses within the microchannels. The numerical model helped to predict the shear stress based on the experimental conditions.

3) Development of in vitro microvessels under controlled conditions. The primary human umbilical vascular endothelial cells were seeded and cultured inside the fabricated microchannel network under a constant perfusion. A confluence monolayer was formed after a few days' perfusion and results showed the cells successfully covered the entire inner surfaces of microchannel networks.

4) Characterize the microvessel network and endothelial cells' responses to flow shear stresses. We examined the junctional formation between endothelial cells (ECs) as an indication of endothelial barrier function. VE-cadherin, an important protein for the maintenance and control of the junctions between endothelial cells, was immune-stained in the entire microchannel. Additionally, the experiments for ECs responses to shear stresses were 
performed and analysis by F-actin microfilaments fluorescent staining. The parameters used for the quantification included percentages of cell alignment along the flow direction, aspect ratios, and cell surface areas.

5) Confocal measurement and quantification of agonist-induced intracellular $\mathrm{Ca}^{2+}$ concentration $\left(\left[\mathrm{Ca}^{2+}\right]_{\mathrm{i}}\right)$ and nitric oxide $(\mathrm{NO})$. Both $\left[\mathrm{Ca}^{2+}\right]_{\mathrm{i}}$ and $\mathrm{NO}$ play important roles in the regulation of a variety of microvessel functions including endothelial barrier function, i.e. microvessel permeability.

\subsection{Significance and novelty}

The research work in this dissertation demonstrated in vitro formation of a microvessel network that recapitulates key features of microvessels in vivo and validated its utility for biological applications. A novel approach to mimic geometries of in vivo microvasculature provided a flexible, cost-effective, and reproducible micromanufacturing method for fabricating multi-diameter microchannel networks with circular cross-sections. This enables the designed microchannel networks obeys the Murray's Law to mimic the geometry and fluidic conditions of branching microvessels in vivo. Additionally, endothelial cells in our model keep their phenotype, viability, proliferation with proper barrier functions, and respond to flow shear force and inflammatory stimuli. Agonist-induced changes in intracellular $\mathrm{Ca}^{2+}$ concentration $\left[\mathrm{Ca}^{2+}\right]_{\mathrm{i}}$ and nitric oxide (NO) production were successfully real-time measured and quantified at individual endothelial cell levels using our model. The results were similar and comparable to those derived from individually perfused intact venules. Comparing to other conventional in vitro models, our model is more physiological realistic. This novel in vitro microvessels model could provide critical insight over conventional models, recapitulate functional microvessels, and mimic the cellular, physical, and hemodynamic 
environment of the microcirculation. It is able to offer valuable quantitative insights into the microvascular signalling and functional studies, curtail high experimental costs and complexities associated with animals and in vivo studies, and provide options to study diseases for which no good animal models exist.

\subsection{Structure of the dissertation}

This dissertation consists of eight chapters. It is initiated with the dissertation overview in Chapter One, in which the motivation, the objectives, significance, and novelty of this research were stated. Followed with chapter one, seven chapters introduced the details of the research work. In Chapter Two, the current microvascular research was reviewed including different techniques and models, the necessaries and advantages of applying microfluidic technologies, and especially for current state of the art microfluidic microvascular models. Chapter Three discussed the importance and challenging in microfluidic based cell culture. Two different perfusion strategies were discussed in details. Chapter Four demonstrated standard methods for fabricating microchannel networks and introduced the fluidic analysis and simulation using COMSOL software. Chapter Five demonstrated a novel method to fabricate multi-diameter circular microchannel network, which mimicked the geometries and fluidic flow conditions of in vivo microvessels. Chapter Six characterized the in vitro growth endothelial monolayer, junction formation for barrier functions, and endothelial cells' responses to different shear flow conditions. Chapter Seven presented the experimental design and testing procedures of confocal measurement of adenosine triphosphate (ATP) stimulated $\left[\mathrm{Ca}^{2+}\right]_{\mathrm{i}}$ and $\mathrm{NO}$ production using the developed microvessel model. Chapter Eight summarized the research work and presented the future work using this developed functional microvessel model. 


\section{Chapter 2 Literature Review}

\subsection{Conventional microvessel models}

\subsubsection{Anatomy of microvessel structures}

The fundamental study regarding microvascular research includes the molecular and cellular bases of endothelial cell structure and function, the role of various molecules and cells in regulation vascular development, physiological regulation of permeability, vascular tone and hemodynamics, and the process of transendothelial transport in relation to endothelial junctions and adhesion molecules. (Shepro 2005) The endothelium, a monolayer of endothelial cells that lines the inner surface of the blood vessels and the lymphatic system is in direct contact with the blood/lymph and the circulating cells. Typical microvascular architecture is shown in Figure 2.1a, where the capillaries branch from an arteriole and join again to form a venule. (Kessel and Kardon 1979)

The endothelial cells are anchored to the basal lamina that is a specialized form of extracellular structure with the thickness of $80 \mathrm{~nm}$. The shape of the endothelial cells varies across the vasculature. In general, the morphology of endothelial cells is a thin and slightly elongated shape. They are around $50-70 \mu \mathrm{m}$ in length, $10-30 \mu \mathrm{m}$ in width, and $0.1-10 \mu \mathrm{m}$ in thickness. (Félétou 2011) Figure 2.1b shows the electron micrograph of a cross section of a small capillary in the pancreas. The wall is formed by a single endothelial cell surrounded by a basal lamina. (Bolender 1974) 
a

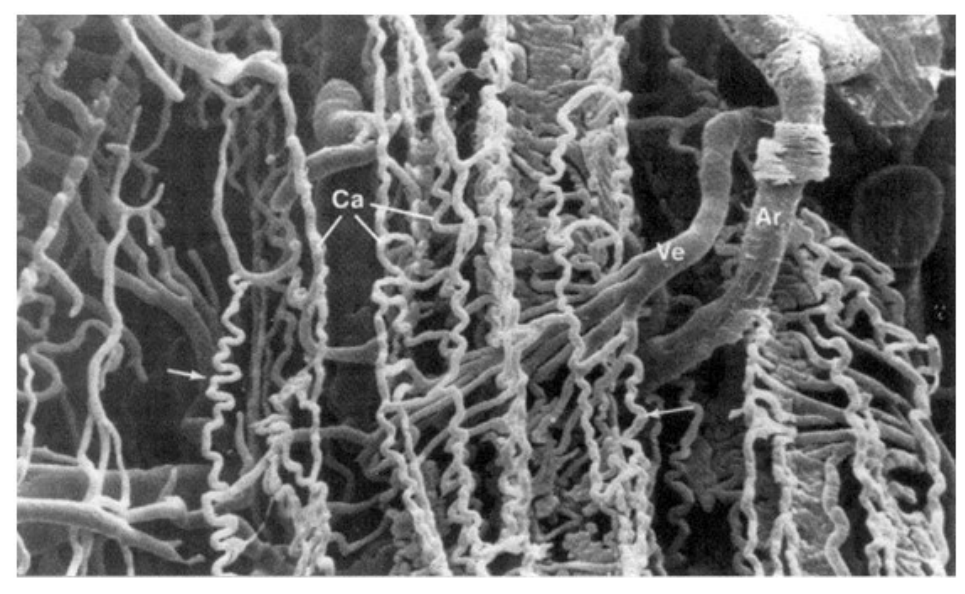

b

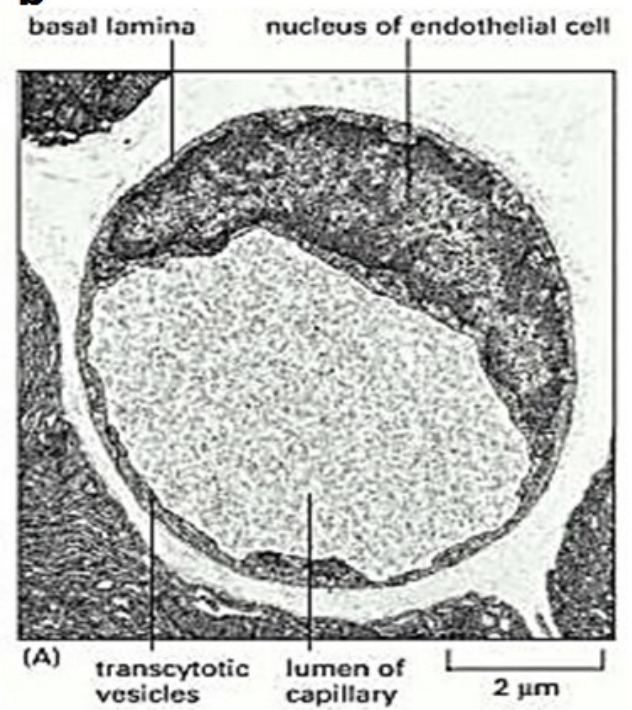

Figure 2.1 a) Microvascular architecture in skeletal muscle. Capillaries (indicated as $\mathrm{Ca}$ ) are oriented longitudinally in the same direction as the muscle fibers they supply. The capillary network is branching out from an arteriole (indicated as Ar) and joining back together to form a venule (indicated as Ve). (Kessel and Kardon 1979) b) Electron micrograph of a cross section of a small capillary in the pancreas. The wall is formed by a single endothelial cell surrounded by a basal lamina. Note the small transcytotic vesicles, which according to one theory provide transport of large molecules in and out of this type of capillary: materials are taken up into the vesicles by endocytosis at the luminal surface of the cell and discharged by exocytosis at the external surface, or vice versa. (Bolender 1974)

\subsubsection{In vivo models}

In vivo approach utilizing animal models is one of the fundamental methods to investigate biological aspects of the microvessels such as vascular permeability, cell signaling, cell-to-cell communication and vascular function, and endothelium dependent regulation of vascular tone. It is also widely used to evaluate new pharmaceutical approach to treat microvascular disease. (Jain, Munn et al. 2011; Vilahur, Padro et al. 2011; Craig, Grau et al. 2012; Stübs, Picker et al. 2013) The in vivo models can be divided into three broad categories based on the preparation method (Jain, Munn et al. 2011): chronic transparent windows (rabbit ear chamber, dorsal skin fold chamber in rodents, and cranial windows in mice and rats), exteriorized tissue or ex vivo model 
(mesentery and cremaster muscle) and in situ preparations (chick embryo chorioallantoic membrane model, Miles assay and zebrafish).

The first transparent window assay was introduced in the 1930s by implantation the device in the ear of a rabbit to study angiogenesis. (Folkman 1972) Later on dorsal skin fold chamber was developed by surgically implanting a titanium frame to support a transparent window on the back of for mice and other rodents to observe tumor growth and microcirculation. (Papenfuss, Gross et al. 1979) Dorsal chamber in mice is one of the most widely used chamber preparation. It has been used for many years and provides chronic access to exposed tissues with small surgical requirements. (Jain, Munn et al. 2011; Laschke, Vollmar et al. 2011) The advantage of these window models include direct access without further surgical treatment and the capability of long term monitoring of microcirculation with intravital microscopy. However, the imaging techniques of transparent window assay are still majorly limited on the surface of the windowed tissue, and only the tissue that can be accessed from outside of the body is available. (Fukumura 2005) For internal organs and tissues, it is necessary to exteriorize them surgically and place them on a microscopic stage.

Rat mesenteric assay is one of the widely used exteriorized or ex vivo tissue model. (Norrby 1998; Dredge, Horsfall et al. 2005; Duansak and Schmid-Schönbein 2013; Kelly-Goss, Sweat et al. 2013) The mesentery is a membranous, virtually translucent tissue between the mesenteric arterial/venous vessels that is feeding the small intestine. It can be exteriorized from the abdomen by a single incision after the rat is anesthetized. (Kelly-Goss, Sweat et al. 2013) The test tissue is natively vascularized, and the microvessel network is almost two-dimensional (2D) due to being extremely thin and can be assessed microscopically in vitro. (Norrby 2011) It is also possible to isolate and cannulate single vessel from the mesenteric tissue as an ex vivo model and 
apply a microperfusion technique to study the biophysics and cell biology of microvascular permeability. (Renkin, Curry et al. 1974; Clough, Michel et al. 1988; Zhou and He 2010) The window assay can be applied to any tissue with variable degree of difficulty, but the duration and frequency of the observation and testing is limited.
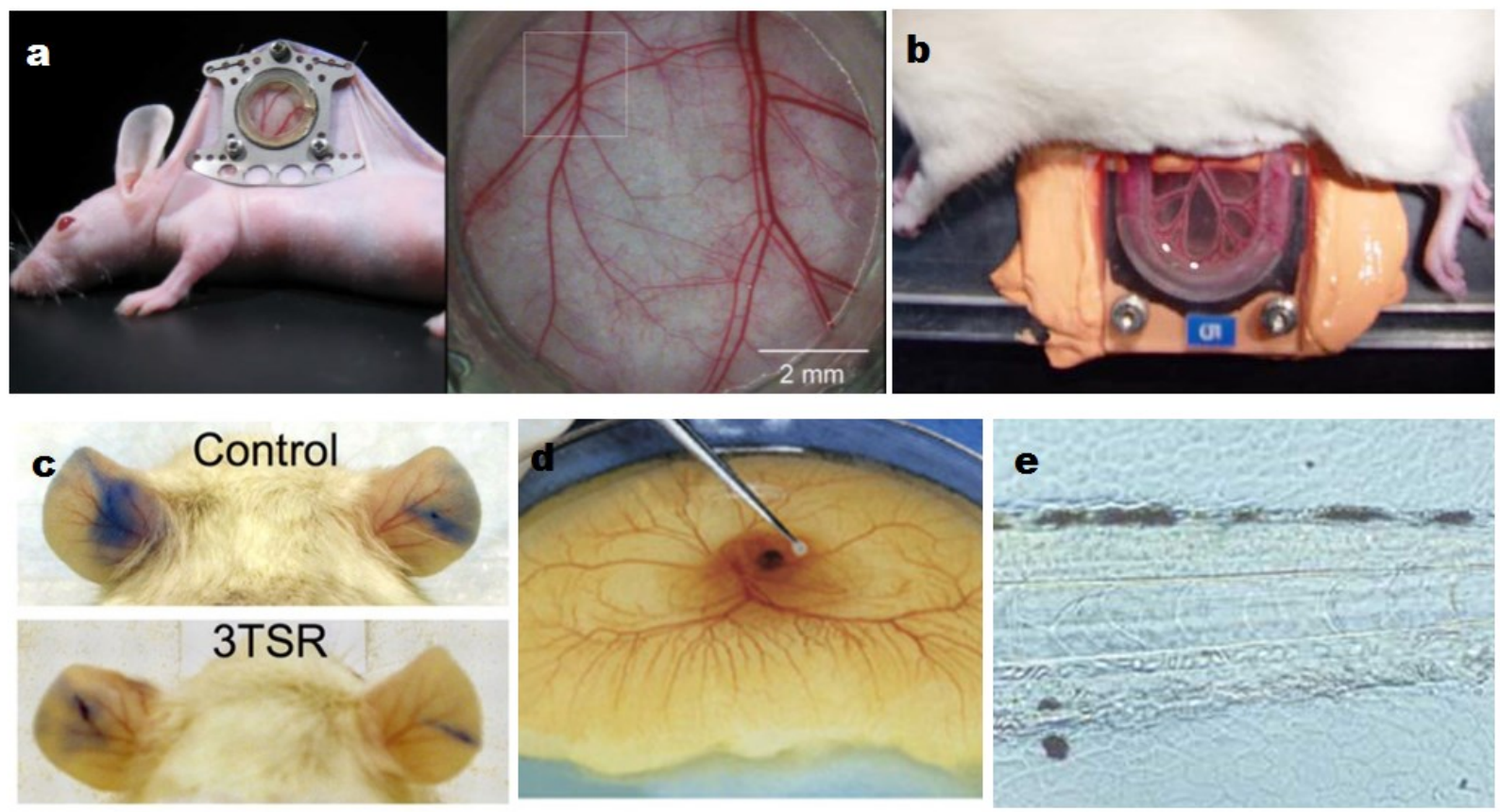

Figure 2.2 a) A dorsal skinfold window chamber model on mice and the enlarged white light microscopy of entire window. (Mariampillai, Standish et al. 2008) b) A typical rat mesenteric window assay. (Dixon 2006) c) Miles assay on the ear of untreated and 3TSR-treated mice. (Zhang, Kazerounian et al. 2009) d) CAM assay is a low-cost method for assessing angiogenic factors in an established vessel system. (Zetter 2008) e) The tale of zebra fish show the ability to discern structures on the cellular level. (Zon and Peterson 2005)

In situ preparations utilize the natural site of animal tissue such as ear and tale of rodents, chick chorioallantoic membrane, and zebra fish to perform microvascular test. Miles assay has been widely used to test the general status of plasma leakage in vivo by tissue staining with pontamine sky blue. (Miles and Miles 1952; Murohara, Horowitz et al. 1998) The Miles assay 
does not require invasive preparation, however as a result the access level to different types of tissue is limited. Chick embryo chorioallantoic membrane has been introduced as a model since the early 1970's. The basic assay is performed by implant a window through a hole cut in the eggshell. After couple of days of incubation, the process of angiogenesis or anti-angiogenesis in vivo can be quantified via image analysis or colorimetric detection methods. (Wilting, Christ et al. 1993; Ribatti, Nico et al. 2001; Tamilarasan, Kolluru et al. 2006) It is difficult to quantify newly formed vessels precisely, as a result, CAM models are mostly used for screening purpose. Zebrafish is a newly addition to the field of microvascular research. The optically clear embryo was first developed as a vertebrate genetic model since the 1970s. (Grunwald and Eisen 2002) Later on, it turns out to be a useful model for vessel formation with very high resolution (Nicoli, Ribatti et al. 2007; Karpanen and Schulte-Merker 2011) and provides experimental analysis of the molecular and cellular mechanisms regarding endothelial permeability and corresponding vascular diseases. (Jeong, Kwon et al. 2008; Dejana, Tournier-Lasserve et al. 2009) However further validations are still required to prove the model whether it is able to reflect the cellular and vascular aspect of mammalian species. (Lai and Lo 2013)

\subsubsection{Conventional in vitro models}

\subsubsection{Static culture model}

Fundamental understandings of microvascular functions rely on the study of the basic building block of microvascular structure, i.e. endothelial cells. The focuses of these models include responses of endothelial cells to relevant biological, chemical, or physical cues by carrying on complex molecular or pharmacological tests. (Franke, Galla et al. 2000; Nakatsu, Sainson et al. 2003; Saleh, Whyte et al. 2011; Takeshita and Ransohoff 2012) 
In conventional in vitro studies, endothelial cells are plated on cell culture dishes, glass coverslips or well plates until cells reach confluent. The cells are subjected to various stimuli and analyzed with different kinds of biological or biochemical techniques. The common applied stimuli include integrin, growth factor, and different types of mediators such as PAF, ATP, ADP, and fibrinogen. Transwell is another highly used in vitro model in which the endothelial cells are plated onto a porous polymer membrane. (Lukacs, Strieter et al. 1995; Shen, Goderie et al. 2004; Tamilarasan, Kolluru et al. 2006)

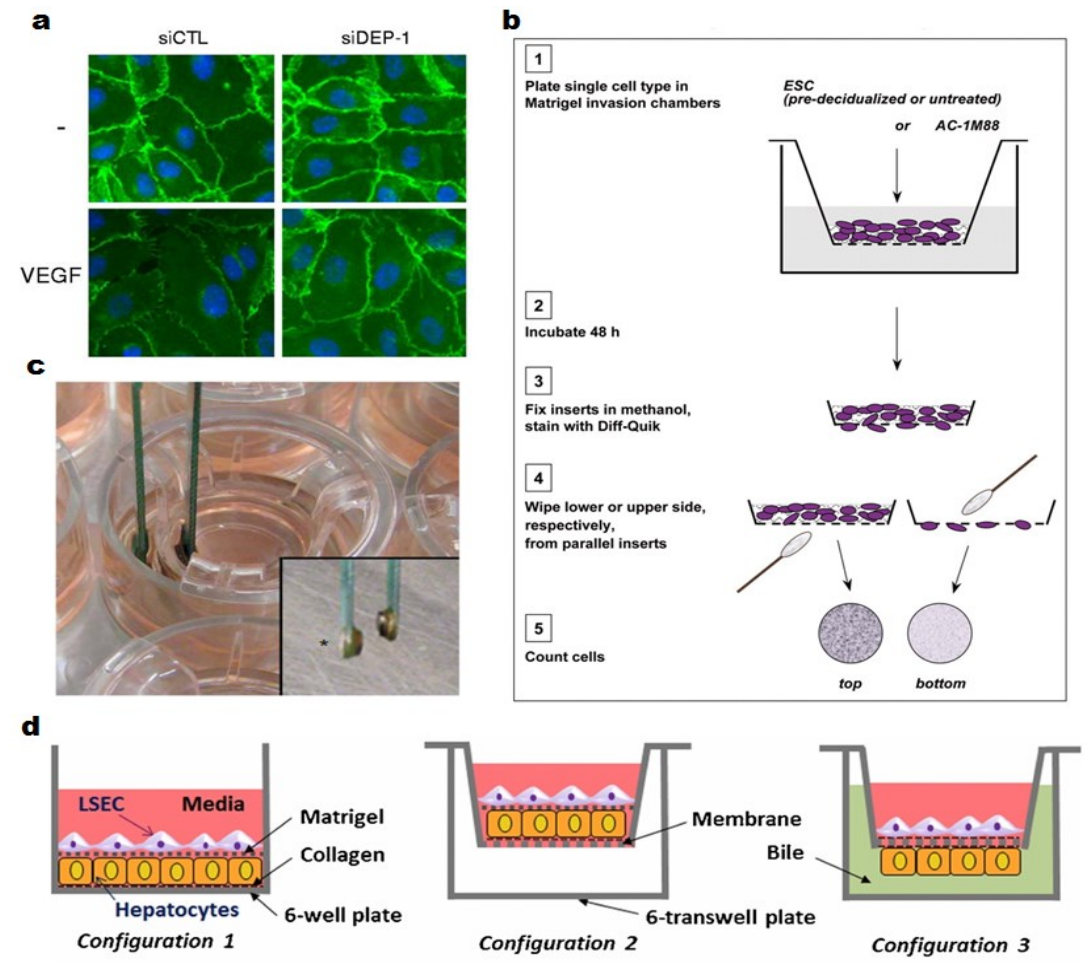

Figure 2.3 a) Endothelial permeability assay performed on transwell system shows VEGFinduced loosening of cell-cell junctions. (Spring, Chabot et al. 2012) b) Scheme of matrigel invasion assay of endometrial stromal cells (ESCs) or AC-1M88 a model for extravillous trophoblast cells. (Gellersen, Reimann et al. 2010) c) Characterization of polarized epithelial cells by measuring transepithelial resistance with a voltohmmeter (Sonoda, Spee et al. 2009) d) Schematic diagram of cell culture platforms to mimic the liver sinusoid. (Kang, Rawat et al. 2013) 
Transwell culture system is easy to handle, provides good cell visibility under optical microscope, and the availability for different surface treatments for different applications such as wound healing, (Chen, Tredget et al. 2008) cell migration, (Kiosses, Shattil et al. 2001) and tumor metastasis. (Brantley-Sieders, Fang et al. 2006) It can also be used as a co-culture model to study the blood-brain barrier, (Franke, Galla et al. 2000) or a permeability assay to study the immune responses (Muller 2003) and molecular transport. (Senger, Perruzzi et al. 2002) Figure 2.3 shows couple examples of transwell systems for microvascular related research. However, the critical constrains of transwell system is that the cultured endothelial cells are usually a $2 \mathrm{D}$ confluence monolayer. Additionally it is unable to apply physiological features such as shear flow over the cell layer.

\subsubsection{Shear flow model}

To apply shear flow to endothelial cells, many in vitro systems were developed to reproduce certain features of in vivo flow. The most common ones include the parallel plate flow chamber, parallel disk viscometer, cone and plate viscometer, orbital shaker, and capillary tube.

The parallel disk viscometer was originally to measure the shear properties of fluids such as viscosity (Yeow, Leong et al. 2008). Because there was linear velocity change at different radial distance, the flow pattern inside parallel disk viscometer was not uniform. (Chiu and Chien 2011) The orbital shaker beard the same problem as shown in Figure 2.4a. Although the flow generated by the orbital shaker was a steady circular flow, the cells cultured in the center of the plate did not show any alignment due to the uneven shear stress distribution. (Mao, Xie et al. 2014)

On the other hand, the cone and plate viscometer and parallel plate flow chamber can yield a considerable uniform shear stress, which is suitable for studying EC response to shear stress in 
terms of molecular signaling and gene expression. Cone and plate viscometer was originally used in tissue culture to study the endothelial cell morphology response to fluid shear stress. (Franke, Gräfe et al. 1984; Spindel, Burke et al. 2014) As shown in Figure 2.4b, a cone plate was fixed on a standard culture plate. By controlling the rotation velocity of the plate, it was possible to generate different flow types within this system. However, it is difficult to precisely align and generate a uniform and steady flow field; and the large variations within the culture plate as the magnitude of the shear stress is depended on cone angle, rotation velocity, and the radius of plate. (Malek, Ahlquist et al. 1995)

The parallel flow chamber assay was widely used due to its simplicity in design, assembly, maintenance and operation. (Peled, Grabovsky et al. 1999; Bernardo, Ball et al. 2004; Conway, Williams et al. 2010; O'Brien, Kent et al. 2012) As shown in Figure 2.4c-d endothelial cells were seeded on a flat substrate such as a glass slide (with surface modification/treatment if necessary). After the cells reached their confluence, the flow chamber was assembled by sandwiching the cell monolayer with a pair of parallel plates, and proper inlet/outlet connections were set on the top plate. The height : width ratio of this kind of design was at least 1:50 to provide a uniform laminar flow across the channel (Morgan and Yarmush 1999). Most widely used flow chamber was a single channel with a rectangular cross-section.

Some modifications have been made such as tilting/curving the sidewalls, varying the chamber height, or applying alternative flow pattern such as Hele-Shaw flow. (Figure 2.4 e-f) (Qin, Jiang et al. 1998; LaMack and Friedman 2007; Plouffe, Radisic et al. 2008; Dolan, Meng et al. 2011) Those designs were introduced to achieve disturbed shear stress, linearly changed shear stress, or constant wall shear stress gradient within the device. 
a

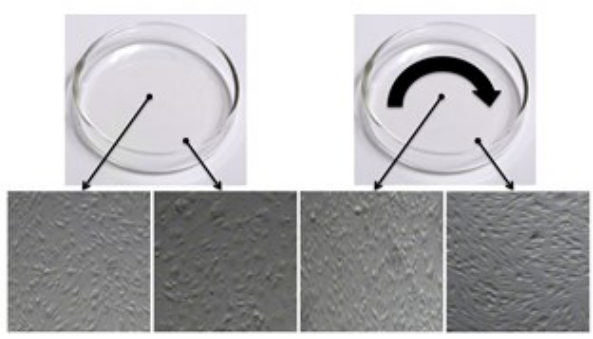

C.

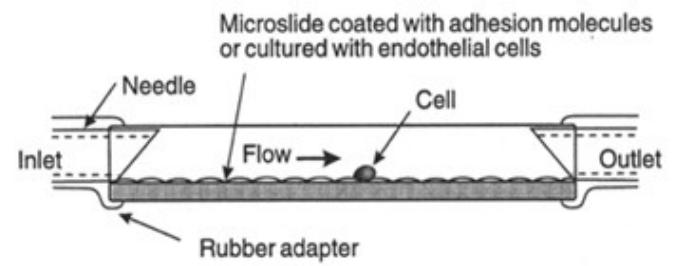

e

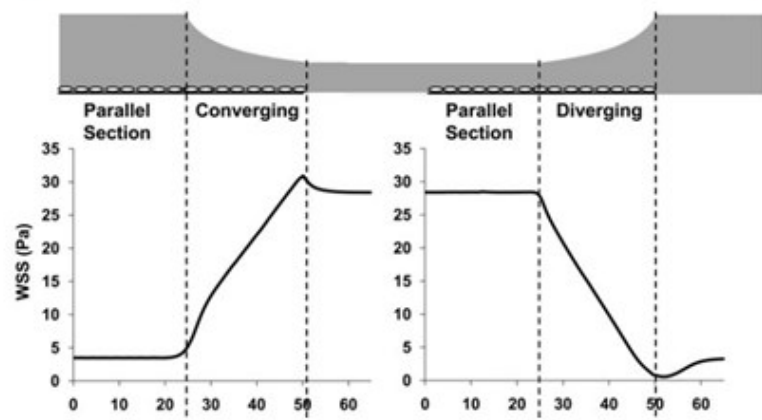

b

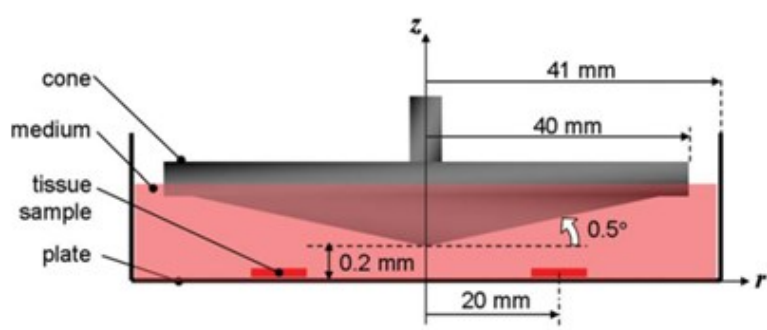

d

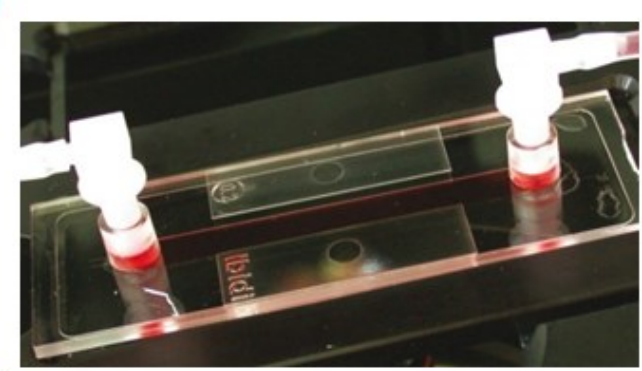

$\mathbf{f}$

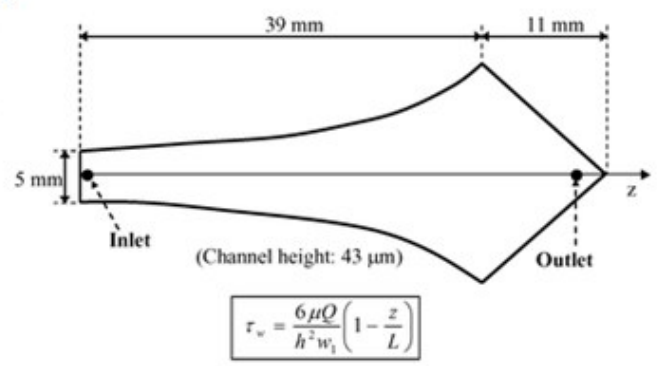

Figure 2.4 a) The cells cultured in the center of the plate did not show any alignment with orbital shaker. (Mao, Xie et al. 2014) b) A cone-and-plate system complying with the dimensional requirements to limit secondary flow effects. (Sucosky, Padala et al. 2008) c) An illustration of the flow chamber. (Cao, Usami et al. 1997) d) The testing set up using a commercialized flow chamber. (Walsh, Murphy et al. 2011) e) The height variation of the flow chamber (showed in cross-section view) would result in a wall shear tress (WSS) variation. (Dolan, Meng et al. 2011) f) Schematic diagram of flow chamber design for a variable shear stress Hele-Shaw device. (Plouffe, Radisic et al. 2008)

The flow chamber is able to seed and collect a large amount of cells after the experiment and used the cells for other experiments such as DNA or RNA extraction, immunohistochemistry, or scanning electron microscopy (Dekker, van Soest et al. 2002; Lane, Jantzen et al. 2012). 
However, this also requires considerable amount of media and reagents during the flow experiment and the following tests (Feng, Fukuda et al. 2011). The other disadvantage of such model is that the single channel geometry limits itself to introduce a more complex, physiologically relevant flow into the system. Furthermore, the endothelial cells are usually seeded at high density and tested within the following 24 hours. It is known that there's transient and long-term cytoskeleton reconstruction under such shear stress change (Galbraith, Skalak et al. 1998). For long-term studies, it would be more preferable to develop a model that already incorporates a hydrodynamic environment capable of excluding transient variation of shear stress that is more close to the in vivo condition, and the capability of exposing the cells to shear stress over a longer period of time.

\subsection{Microfluidic model for microvascular research}

Capable of manipulating small amount of fluid flows in micro or sub micro scale, microfluidic devices are able to provide a more efficient and lower cost solution for developing an in vitro model for microvascular research. Comparing with the conventional in vitro assays, the advantages of microfluidic based devices are: cost efficient; using very little amount of cells and reagents; able to provide well controlled hydrodynamic environment; provide in vivo vascular geometries and features such as channel size in micro-scale, branching structure, shear flow, gradient generation, and 3D scaffold for cell seeding. It is also possible to integrate microfluidic devices into well-established in vitro microscopic system for real-time and longterm imaging/monitoring. These models with sufficient complexity may work as artificial surrogates for curtailing the high experimental costs and complexities associated with animals and in vivo studies. 


\subsubsection{Materials used for microfluidic device fabrication}

The soft lithography fabrication method is suitable to develop low cost, high resolution, rapid prototyping devices. It is able to use transparent material to assemble microchannel devices with versatile design such as parallel/series of channels, multilayer of channels, and network design with bifurcations and junction that are ideal for biomedical research and applications. (Rogers and Nuzzo 2005) Many microfluidic devices have been developed for endothelial cell related research (El-Ali, Sorger et al. 2006; Ibrahim and K Richardson 2013; Raynaud, Butler et al. 2013; Reymond, d'Água et al. 2013). Among those, the most widely used material is an elastomeric material polydimethylsiloxane (PDMS). PDMS is an optically transparent, nontoxic, gas permeable soft elastomer that has become the most widely used building material for many microfluidic applications since its introduction. (McDonald and Whitesides 2002; Whitesides 2006) PDMS is a material that has offered many advantages for fabricating microfluidic devices.

There are other polymers available such as polyimide, poly (methyl methacrylate), polystyrene, cyclic olefin copolymer. Biological gel has also been introduced as the building material to create microvascular network in vitro such as collagen (Tang, Golden et al. 2004; Golden and Tien 2007; Zheng, Chen et al. 2012), alginate (Lee, Shin et al. 2009; Hatch, Hansmann et al. 2011), polyethylene glycol diacrylate (HwanáSung 2011) and gelatin (Nichol, Koshy et al. 2010).

\subsubsection{Microvascular cell culture and testing}

One advantage of microfluidic microchannel devices is the increased efficiency by performing tests in a series of parallel channels. As shown in Figure 2.5a, the microfluidic parallel channels were fabricated with soft lithography techniques to study the platelet adhesion under shear flow conditions. (Kantak, Gale et al. 2003) 
$\mathbf{a}$

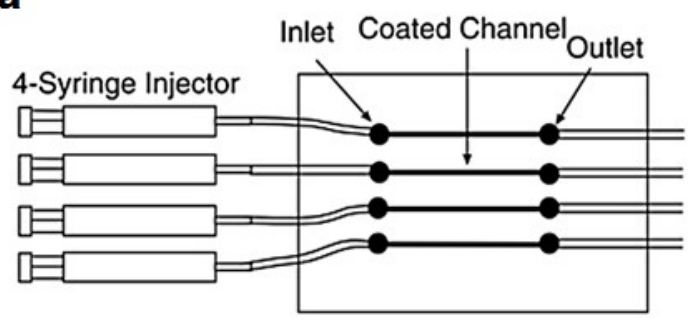

C

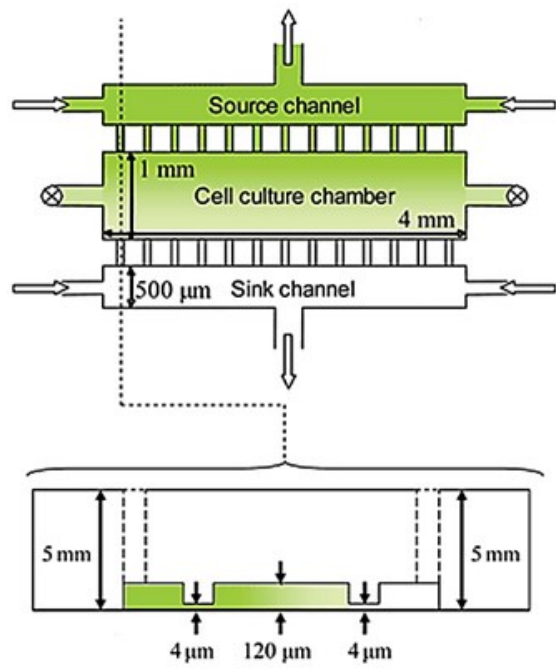

b
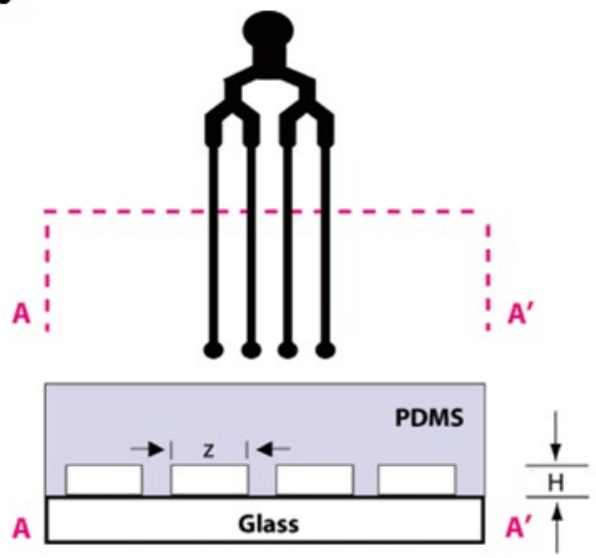

$\mathrm{H}=25 \mu \mathrm{m}$

$\mathrm{z}=500 \mu \mathrm{m}$

d

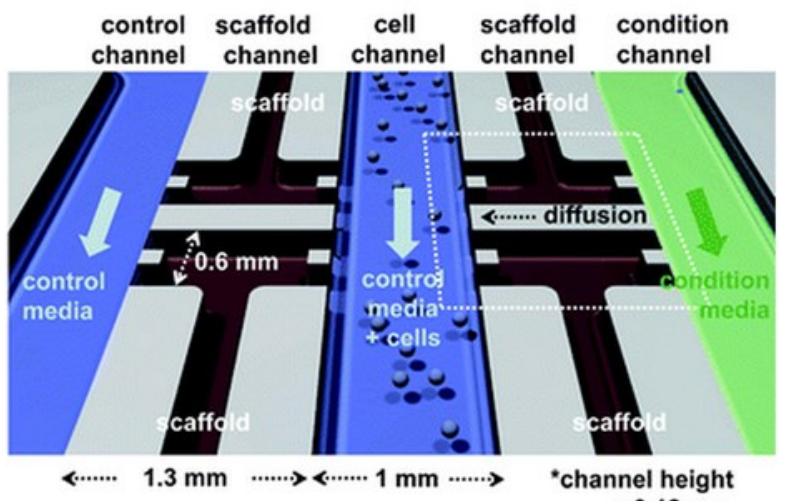

$=0.12 \mathrm{~mm}$

Figure 2.5 a) Parallel microchannels with syringe injectors to provide a controlled flow rate. (Kantak, Gale et al. 2003) b) Short-term adhesion multisampling device accommodating identical channels. (Lu, Koo et al. 2004) c) Schematic of three-chamber microfluidic device. Top view shows positions of reagent channels and cell culture chamber. Microcapillaries with the width of $5 \mu \mathrm{m}$ connect the reagent channels to the cell culture chamber. On the cross-section view, the thickness of each layer is marked where the dashed lines show positions of the inlets and outlets at both ends. (Shamloo, Ma et al. 2008) d) Schematic for microfluidic cell migration assay base on multi-chamber channel with a hydrogel region. In this set-up, it is possible to compare the cell migration behavior between the condition and control sides directly. (Chung, Sudo et al. 2009)

The design of microfluidic channels was improved by using a single inlet to feed four parallel channels to avoid multiple syringe injections. (Figure 2.5b)(Lu, Koo et al. 2004) Later on 
similar design was applied for vascular endothelial adhesion assay with eight parallel daughter channels. (Young, Wheeler et al. 2007) After endothelial cells were seeded into the proteins coated microchannel for 2 hours, a shear flow was induced to test the cell adhesion strength. This kind of short-term cell seeding was arguably fine for adhesion assay. However, for other applications a longer culture period was required. A cell culture chamber with two reagent channels was developed for endothelial chemotaxis study and the cells were kept up to 3 days. As shown in Figure 2.5c, channels were interconnected with microcapillaries. (Shamloo, Ma et al. 2008) This design was further developed into a system in combination with gradient and flow control to study endothelial cell migration and capillary morphogenesis. (Figure 2.5d). Hydrogel was integrated into the PDMS device to promote cell attachment and cell growth. It was also possible to co-culture multiple cell types for cell communication or tumor cell induced angiogenesis study with this kind of microfluidic devices. (Chung, Sudo et al. 2009; Shin, Han et al. 2012; Zervantonakis, Hughes-Alford et al. 2012)

\subsubsection{In vitro microvessel networks}

There are two major aims for the development and culture of in vitro microvessel structure. The first aim is to incorporate microvasculature into artificial tissues. The major limitation of constructing an artificial living tissue is vascularity, (Kannan, Salacinski et al. 2005) as the absence of vascular networks is a major limiting factor for oxygen, nutrition and wastes delivery within any tissue which is over $200 \mu \mathrm{m}$ in thickness. (Tong, De Lagausie et al. 1992; Carmeliet and Jain 2000) The other goal is to develop an in vitro model, for example, an integrated biochip/bio-reactor that can be utilized for endothelial cell related physiological and pathological research such as angiogenesis, vasculogenesis, cell migration, and apoptosis etc. (Van Kooten, Schakenraad et al. 1992; Bouïs, Hospers et al. 2001; Lehle, Straub et al. 2010; Nguyen, Stapleton 
et al. 2013). Comparing with the conventional cell culture models, those advanced microvessel models are able to provide a more controllable condition with specific physiologically relevant features. Figure 2.6 shows microvessel network structure developed in microfluidic devices. Hydrogel was used as scaffolds for endothelial cell seeding as shown in Figure 2.5a. (Golden and Tien 2007) The biological gel can provide a three-dimensional (3D) scaffold for the cell seeding, promote cell adhesion, or serve as a physical barrier to induce chemical gradient within the microchannels. However, the hydrogel is very vulnerable to dehydration and has relatively weak mechanical properties that can easily be damaged by stress or pressure. In a hydrogel integrated PDMS device, 3D lumens were developed through ECM hydrogels that were lined with endothelial monolayers. (Figure 2.6b)(Bischel, Young et al. 2013)

Comparing with the hydrogel devices, the hydrogel/PDMS hybrid devices show longer durability and reduce the dehydration and evaporation of the solutions. The fabricated hydrogel region can still promote cell attachment, and the PDMS microchannels are able to provide better mechanical support. Based on the currently knowledge of angiogenesis and vasculogenesis, perfusable 3D microvascular networks on a chip was developed with showing strong barrier function and long-term stability. (Figure 2.6c)(Kim, Lee et al. 2013) However, a carefully designed microchannel networks with less randomness are more preferred when it is necessary to quantify and precisely control the flow field and corresponding shear stress. Figure $2.6 \mathrm{~d}$ shows an engineered microvascular networks in collagen. (Zheng, Chen et al. 2012) It is also possible to develop a simple, single-mask microfabrication process combined with standard endothelial cell culture techniques to fabricate a microvascular-sized fluidic system that incorporates cultured endothelial cell monolayer that covers the entire 3D inner surface of the microfluidic system. (Figure 2.6e)(Tsai, Kita et al. 2012) 

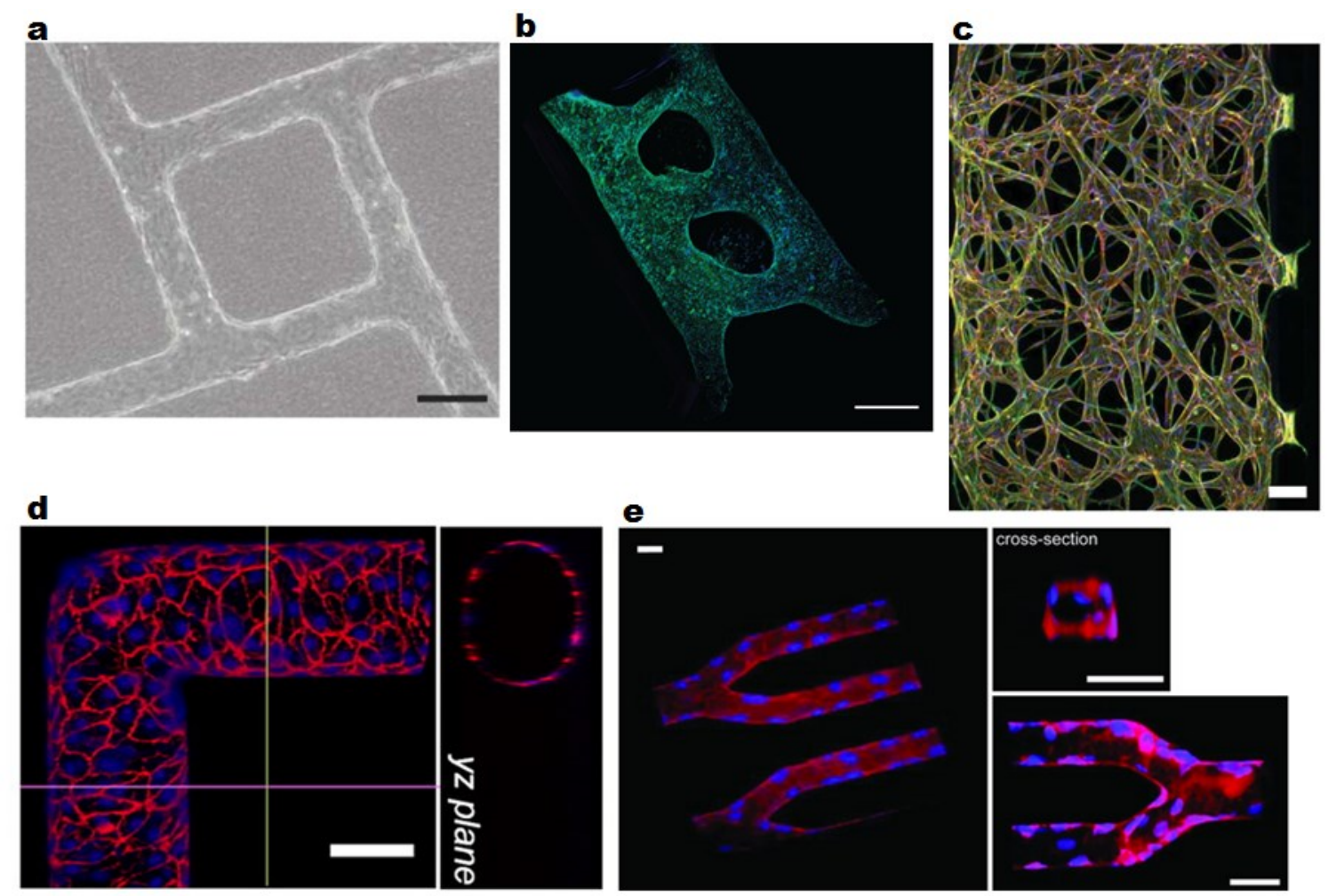

Figure 2.6 a) Use of perfused microfluidic gels as scaffolds for cell culture. High magnification image shows collagen gel with a monolayer of endothelial cells lining. Scale bars: $200 \mu \mathrm{m}$. (Golden and Tien 2007) b) A three-dimensional endothelial-lined lumens were patterned in collagen filled microchannels using basic fluid principles such as passive pumping and viscous fingering of fluids with different viscosities. Scale bars: $500 \mu \mathrm{m}$. (Bischel, Young et al. 2013) c) Engineered 3D microvessels characterized by immunofluorescence: confocal micrographs showing the overall architectures of vascular networks established by vasculogenesis. Scale bars: $100 \mu \mathrm{m}$. (Kim, Lee et al. 2013) d) Confocal sections of endothelialized microfluidic vessels of

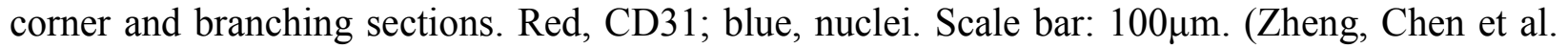
2012) e) 3D renderings of multiple confocal microscopy using fluorescent cell membrane (red) and cell nuclear (blue) dyes show that the endothelial cells line the entire inner surface of the microfluidic channels. Scale bars: $30 \mu \mathrm{m}$. (Tsai, Kita et al. 2012)

\subsubsection{The application of microfluidic microvascular model}

The microfluidic technology has been developed into advanced in vitro microvascular models to enable the studies of vascular related physiological and pathological phenomena such 
as angiogenesis, vasculogenesis, cell migration, cell adhesion and integrin, vessel aging and apoptosis etc. (Van Kooten, Schakenraad et al. 1992; Bouïs, Hospers et al. 2001; Aitkenhead, Wang et al. 2002; Lehle, Straub et al. 2010; Nguyen, Stapleton et al. 2013).

With the addition of perfusion system, microfluidic microvascular models were used to study endothelial cell alignment and elongation (Song, Cavnar et al. 2009; Van der Meer, Poot et al. 2010), cell adhesion (Song, Cavnar et al. 2009), nitric oxide (NO) production (Oblak, Root et al. 2006) and reactive oxygen species (ROS) (Chin, Yu et al. 2011) under different condition of shear stress. The following sections focus on the main function of endothelium: microvessel barrier function, as it is the main application of our microvessel model.

\subsubsection{Microvessel barrier function}

Microvascular permeability can be defined as the capacity the microvascular wall to allow the flow of ions, fluid, and nutrients or even cells such as lymphocytes in and out of the microvessels. (Shepro 2005) As shown in Figure 2.7, small molecules (such as albumin and the ones with size $\leq 3 \mathrm{~nm}$ ) are transported through endothelial cells with a system called vesicular vacuolar organelles (VVOs). The cells and macromolecules that are larger than $3 \mathrm{~nm}$ use the paracellular route which is mediated by cell-cell junctions (such as tight junction and adherens junction). (Azzi, Hebda et al. 2013) The microvascular barrier function is essential to support the viability and function of surrounding tissues. The dysregulation occurs in many disease states or injured conditions, such as inflammation, trauma, diabetes mellitus, thrombosis, and metastatic

tumor development. The endothelial barrier dysfunction can be characterized by hyperpermeability and clinical manifests such as edema. (Yuan and Rigor 2010) 


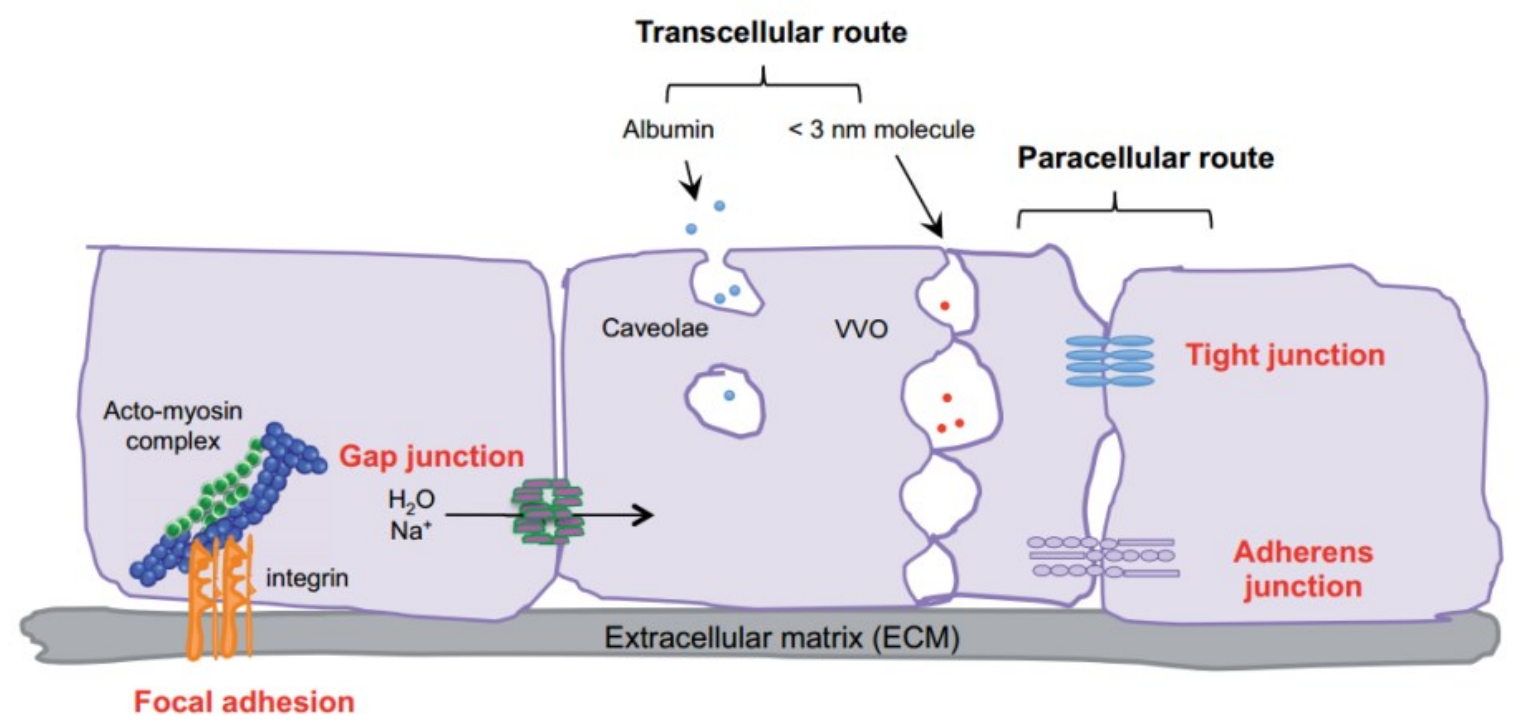

Figure 2.7 The passage of cells and macromolecules through the endothelial barrier can occur through transcellular (vesicular vacuolar organelles) or paracellular (tight and adherens junctions) pathways. Gap junctions ensure water and ion transport. Moreover, endothelial cells are anchored and connected to the extracellular matrix (ECM) through integrin-based adhesion complexes, namely focal adhesions. (Azzi, Hebda et al. 2013)

Many methods have been developed to evaluate the endothelium permeability. In animal models, the experiments were performed by flowing solution such as filtered blood plasma under controlled fluid pressures through microvascular tissue region such as limb, lung or isolated perfused microvessel. The barrier function can be quantified by weight/velocity measurement of filtered fluid, detection of the pressure drop between input and output after the tissue weight reached steady state under a constant perfusion, or measurement of the fluxes of labeled macromolecules/fluorescent tracer across the microvessel wall. (Yuan and Rigor 2010) To assess the permeability in cultured endothelial cells, many in vitro studies of endothelial permeability were performed under static culture conditions. The endothelial monolayer was usually cultured in transwells and characterized with immunofluorescence staining, western 
blotting (Rabiet, Plantier et al. 1996), fluorescence microscopy (Jho, Mehta et al. 2005) and spectrophotometry (van Nieuw Amerongen, Draijer et al. 1998).

However, flow condition, which is missing in static culture conditions, is a very important factor for EC permeability study. It was reported that the transportation of macromolecules such as albumin through the endothelial barrier was shear dependent. (Jo, Dull et al. 1991; Tarbell 2010). Shear stress was also known to modify inter-endothelial junctions and the interaction between cells and extracellular matrix. (Davies, Mundel et al. 1995; Ingber 2002) Many groups have studied the specific intracellular signaling of barrier property change under shear stress such as inositol trisphosphate, activation of Rac, caveolin-1 phosphorylation, and tyrosine kinases such as FAK. (Li, Haga et al. 2005; Mehta and Malik 2006) Young et al. developed a microfluidic membrane chip to measure the endothelial permeability in real-time (Young, Watson et al. 2010). A polyester membrane was sandwiched between two microchannels and primary porcine aortic endothelial cells (PAECs) were seeded onto the membrane. The permeability was measured with the method of spectrophotometry by flowing fluorescein isothiocyanate-labeled bovine serum albumin (FITC-BSA) into the top channel, and detecting the FITC-BSA that passed through the cell layer and went into the bottom channel with laser induced fluorescence microscopy. Their experiment set-up was able to precisely control the flow-induced shear stress and detect the permeability value change. However due to the lack of a fully confluent monolayer there was discrepancy between their permeability model and the previous in vitro models such as transwell systems. There were similar devices reported by different groups with alternative fluorescence probes such as FITC -albumin (Shao, Wu et al. 2009) and fluorescence conjugated dextran. (Zervantonakis, Hughes-Alford et al. 2012) Douville et al. embedded electrodes within the membrane sandwiched PDMS microsystem to take 
transendothelial electrical resistance (TEER) measurement (Douville, Tung et al. 2010). The drawback of their device was that the measurement was performed under static condition, and the potential impact of fluid flow was unknown. The TEER measurement was also introduced to characterize the blood-brain barrier within a microfluidic model by other groups (Cucullo, Couraud et al. 2007; Booth and Kim 2012). Griep et al. applied shear stress to the EC monolayer for 18 hours and studied the dynamic TEER data that can be affected by biochemical stimulation such as tumor necrosis factor alpha (Griep, Wolbers et al. 2013). In those devices, the evaluation of endothelial permeability was performed on a $2 \mathrm{D}$ cell monolayer. Due to the low electrical resistance of in vitro cultured endothelial cells, it was hard to detect subtle resistant change of the cell monolayer. (Shao, Wu et al. 2010)

Other than the solute flux assays and TEER measurement, other factors can be used to indicate physiological changes in endothelial permeability. For example, it has been reported that the initial increase of endothelial intracellular $\mathrm{Ca}^{2+}\left(\left[\mathrm{Ca}^{2+}\right]_{\mathrm{i}}\right)$ can activate downstream pathways to reorganize cytoskeletal protein and dissemble VE-cadherin junctions, and result in the increase of endothelial permeability. (Steinberg and McNutt 1999; Dudek and Garcia 2001) The $\mathrm{Ca}^{2+}$ can also activate the nitric oxide (NO) production, and further increase the endothelial gap formation, which was the main transport pathway in response to fluid and macromolecules during inflammation. (Michel and Curry 1999)

\subsubsection{NO detection}

NO can be released from the local endothelium under shear stress, hypoxic conditions, or in the presence of agonists such as ATP. The releasing of NO can result in a cascade of signaling events that leads to vasorelaxation (Hulvey and Martin 2009). This free radical compound is able to react quickly with oxygen and metals. As a result, it has an in vivo half-life of only a few 
milliseconds in blood, (Thomas, Liu et al. 2001) which presents a considerable technical challenge to performing direct measurements. It is now clear that there are many factors capable of indirectly stimulating NO production in the endothelium. Unfortunately, differentiating the source of NO is challenging in vivo because many cell types are capable of producing and releasing this small, gaseous molecule.

Among various microfluidic based NO detection devices developed by different groups, the detection methods can be characterized into two different types: electrochemical detection, (Spence, Torrence et al. 2004; Cha, Tung et al. 2010) and fluorescence microscopy. (Ranjan, Xiao et al. 1995) Hulvey and Martin integrated barbon ink microeletrodes into a microchip to measure the NO release from bovine pulmonary endothelial cells (bPAECs) that were seeded inside a reservoir connected with a microchannel. (Hulvey and Martin 2009) Kotsis and Spence cultured the bPAECs to the lumen of microbore tubing and detected endothelium-derived NO with a glassy carbon working electrode modified with Nafion in a parallel flow chamber. (Kotsis and Spence 2003) Although those devices showed near real time signal detection, due to the extremely short life time of endothelial derived NO, the sensitivity was hinged due to the considerable physical distance between the electrode and cells. Jiang et al. fabricated the grapheme-hemin sensors for the NO detection where the HUVECs were directly seeded onto the grapheme-hemin electrodes. (Jiang, Cheng et al. 2013) The sub-nanomolar sensitivity they reported was impressive. However, the $\mathrm{SiO}_{2}$ substrate they utilized within a PDMS well was not ideal for endothelial cells attachment. In addition, it encountered the similar problem to integrate such kind of sensor into microchannel devices, as there will be difficulty to achieve a close contact between detector and the region of interest. Fluorescence based detection, on the other hand, has already been widely used by the biologists and physiologists (Navarro-Antolín, López- 
Muñoz et al. 2001; Zhou, Yuan et al. 2013). The fluorescent indicator such as diaminofluoresceins (DAFs) is reliable, highly sensitive, and capable of real time imaging (Kojima, Nakatsubo et al. 1998; Kojima, Urano et al. 1999). Qiu et al. cultured bovine aortic endothelial cells (BAECs) within microcapillary tubes and the NO production under shear stress was detected with an inverted epifluorescence microscope. Oblak et al. cultured bPAECs within a single PDMS microchannel and observed endothelial NO production via dynamic fluorescence microscopy. (Oblak, Root et al. 2006) Similar work has also been done by Letourneau et al. to study the effects of estradiol on NO stimulation of bPAECs. (Letourneau, Hernandez et al. 2010)

\subsubsection{Endothelial intracellular $\mathrm{Ca}^{2+}$ concentration $\left(\left[\mathrm{Ca}^{2+}\right]_{\mathrm{i}}\right)$}

Although there have been many in vitro models for $\left[\mathrm{Ca}^{2+}\right]_{\mathrm{i}}$ related studies, (Carafoli 1987; Thayer and Miller 1990; Zadeh, Glass et al. 2008; Burgazli, Aydogdu et al. 2013) most of the researchers performed the experiments using macro-scale platform (Tran, Farinas et al. 2005) with different cells such as animal cells, (Yin, Zhang et al. 2007) and immortalized cell lines other than primary human endothelial cells. (Wheeler, Throndset et al. 2003; Xu, Li et al. 2010) In microfluidic systems, there are limited reports in the $\left[\mathrm{Ca}^{2+}\right]_{\mathrm{i}}$ measurement using flow chamber. (Corson, James et al. 1996; Melchior and Frangos 2012) However, there are lack of reports in quantifying agonist induced endothelial $\left[\mathrm{Ca}^{2+}\right]_{\mathrm{i}}$ in microchannel network devices. We applied the method developed in individually perfused intact microvesels and quantitatively measured the change of $\left[\mathrm{Ca}^{2+}\right]_{\mathrm{i}}$ after exposed to microvessel model to ATP.

\subsection{Chapter summary}

In summary, the conventional in vivo and in vitro models have their own strength and weakness. In animal models, the whole body physiology and interacting organ systems are available. The immune system, a real vasculature, and the variety of cell types are essential for 
many studies such as permeability, inflammation, and cell-cell interaction. The animal model also replicates the clinical situation, as the test drug or stimuli can be induced by injection, inhaling or oral treatment. The responses of all the metabolic, cellular, and molecular alterations can be quantitatively assessed. The cell culture model on the other hand, is less expensive, less labor intensive, and easier to manipulate (Little, Mironov et al. 1998; Stephenson, Banerjee et al. 2004; Sukardi, Chng et al. 2011). In many cases, cultured endothelial cells are the only viable models for testing mechanistic hypothesis related to cell signaling pathways. (Yuan and Rigor 2010) The main issue of in vitro models is the physiological relevance. As the endothelial cells in those models are cultured on a flat substrate to form a homogenous monolayer, they do not experience a complex microenvironment as what happens in in vivo condition. Comparing with the conventional macro-scale cell culture method, microfluidics is able to provide a more physiological relevent endothelial culture platform. Microfluidics has the advantage to provide a solution that has not been addressed by those macroscale approaches, such as developing a more physiologically relevant in vitro model. In this dissertation, an in vitro functional microvessels was developed to provide a platform for the study of complex vascular phenomena. In the following chapters, the in vitro formation of a microvessel network will demonstrate the successful recapitulations of key features of microvessels in vivo, and the validation of its utility for biological applications. 


\section{Chapter 3 Microfluidic Based Cell Culture}

The advantage of microfluidic devices is the capability to constantly replenish nutrients and remove waste product from cultured cells in a timely manner. However, it is still challenging to provide a controlled, stable, and constant long-term perfusion for certain applications such as microvascular research, in which the endothelial cells or co-cultured multi cell types require at least few days of culturing and proliferation, followed by tests that last for several hours up to days. Furthermore, it is even more difficult to conduct dynamic control of flow patterns to achieve the required shear stresses. In this chapter, the current available perfusion technique will be briefly reviewed. Then based on the flow rate requirement for our microvessel model, two long-term perfusion techniques were applied. An optimized syringe pump system was finally used for endothelial culture as well as shear stress test under different shear stress.

\subsection{Background}

\subsubsection{Conventional 2D and 3D culture}

The conventional macro-scale in vitro cell culture methods apply a static culture condition over cell monolayer on a 2D flat surface, such as flask, culture dish, and multi-well plates. Although static cell cultured in multi-well microplates or petri-dish are easy to operate and already standardized over 100 years, this approach lacks the ability to mimic in vivo microenvironment for microvascular research. The cells in native tissues are subjected to various biological factors such as signaling molecules, growth factors, hormones, or cytokines. Under static culture condition, it is very hard to generate uniform/transient mechanical or chemical stimuli (like signaling molecules or inflammatory factors) that are important for the in vivo cell functions. (Huh, Hamilton et al. 2011; Bhatia and Ingber 2014) It has also been well recognized 
that the importance of flow and the associated wall shear stress in regulating endothelial biology, such as by inducing changes in cell shape and orientation, secretion and organization, proliferation and differentiation, intracellular signaling, cytoskeleton protein production and gene expression, vessel maturation and structure, and barrier functions (Chien, Li et al. 1998; Nerem, Alexander et al. 1998; Li, Haga et al. 2005; Chau, Doran et al. 2009; Lee, Vunjak-Novakovic et al. 2009; Lee and Niklason 2010; Potter, Lundberg et al. 2011). As shown in Figure 3.1a, the endothelial cells under static culture lacked elongation and alignment. When the cells were subjected to physiological levels of shear stress, their cytoskeleton structure aligned to the direction of flow as shown in Figure 3.2b. (van der Meer, Poot et al. 2009)
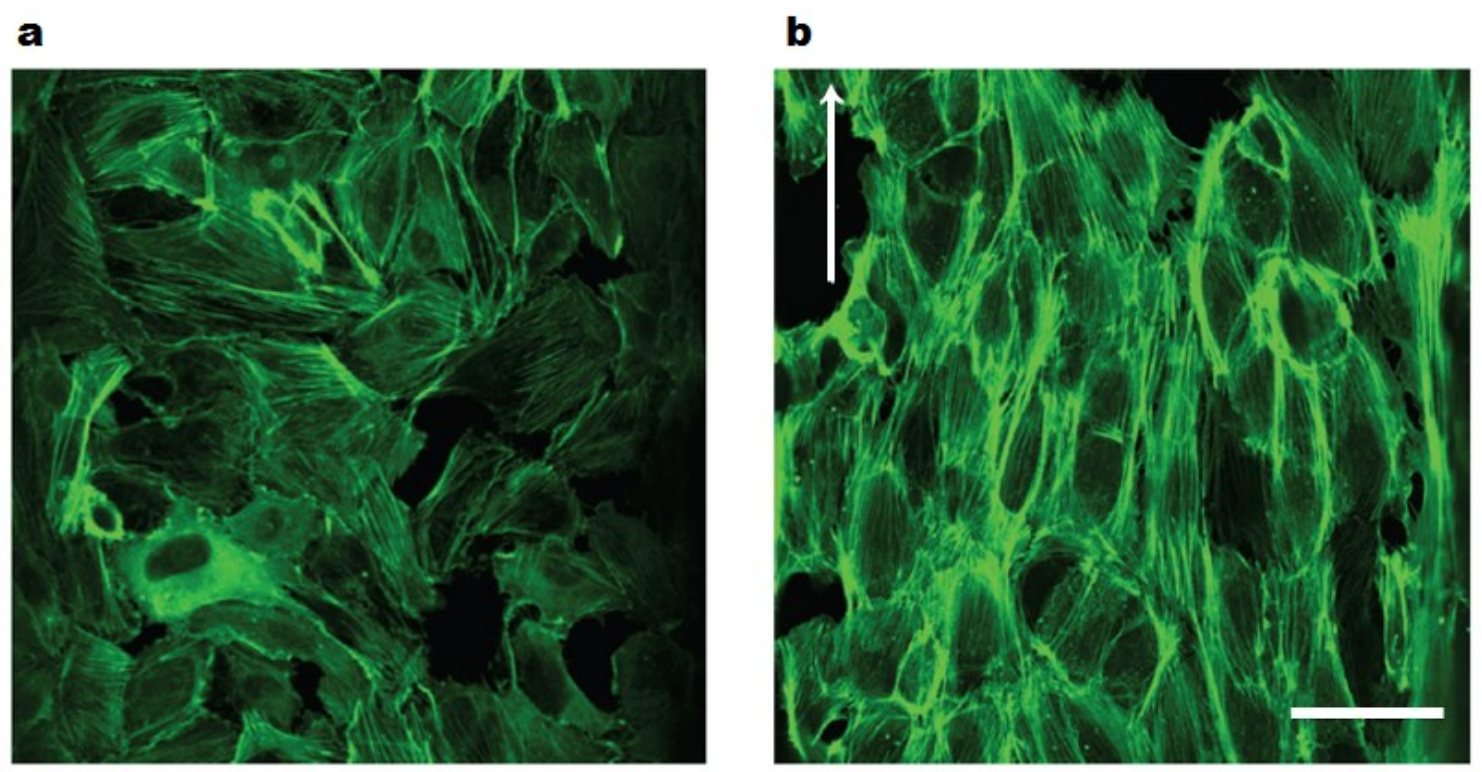

Figure 3.1 Cytoskeleton reorientation of endothelial cell under shear stress. a) HUVECs were cultured under static condition. When reaching confluence, the cells were fixated with paraformaldehyde and stained for actin filaments (green). b) After subjected cell to shear stress, most filaments were aligned and oriented in the flow direction that was indicated by the arrow. Scale bar: $50 \mu \mathrm{m}$. (van der Meer, Poot et al. 2009)

To provide a better approximation of the in vivo conditions, 3D cell culture models have been developed by composing either natural extracellular matrix (ECM) with synthetic polymers. 
Cells were cultured inside the hydrogels and formed by self-organization with or without mesenchymal cells in extracellular matrix (ECM) (type I collagen, fibrin, or matrigel) (Kanzawa, Endo et al. 1993; Donovan, Brown et al. 2001; Davis, Bayless et al. 2002; Velazquez, Snyder et al. 2002). Comparing with 2D models, these 3D models were useful for studying the molecular basis of tissue function and better captured the signal pathways in some diseased states. However, the artificial vessels formed through a random process of morphogenesis that lacked the desired spatial reproducibility and orientation. Additionally, the impact of flow on microvascular stability remained largely unknown because these self-assembled vessels do not easily combine luminal flow with a 3D tubular organization (Tang and Conti 2004), posing a challenge to engineering blood vessels to have barrier functions and long-term vascular stability.

\subsubsection{Microfluidic cell culture}

Microfluidic devices offer the possibility to overcome the issues mentioned above. As shown in Table 3.1, microfluidic based cell culture can provide a dynamic real-time control of very small volumes of fluid. It is possible to perform drug/protein stimulation and microscopic imaging simultaneously. It is also a cost-efficient solution with the capability of high automotive and parallelization for biochemical and biological analysis. (Takayama, McDonald et al. 1999; Cho, Schuster et al. 2003) For microvascular research, culture endothelial cells in continuously perfused microchannels enable a physiological relevant microvessel model and preserve key functions of the in vivo microvasculature. (Mehling and Tay 2014) Due to the small dimensional features, the fluid flow within the microfluidic device is usually laminar flow. The diffusive property of laminar flow is able to provide the precise placement of small molecules at subcellular level that is currently not achievable on a macro-scale. (Takayama, Ostuni et al. 2001) 
Table 3.1 Basic requirements for cell culture, and improvements when microfluidic methods are used. (Mehling and Tay 2014)

\begin{tabular}{|l|l|l|}
\hline Requirements & Conventional cell culture & Microfluidic cell culture \\
\hline $\begin{array}{l}\text { Control of temperature and } \\
\text { gas }\end{array}$ & $\begin{array}{l}\text { Large fluid volumes prevent } \\
\text { fast exchanges }\end{array}$ & $\begin{array}{l}\text { Small volumes allow dynamic } \\
\text { control }\end{array}$ \\
\hline $\begin{array}{l}\text { Addition of nutrients and } \\
\text { removal of metabolites }\end{array}$ & $\begin{array}{l}\text { Infrequent, manual exchange } \\
\text { of large volumes }\end{array}$ & $\begin{array}{l}\text { Well controlled continuous } \\
\text { exchange of media }\end{array}$ \\
\hline Real-time imaging & Mostly not feasible & Feasible \\
\hline Parallelization & Not feasible & Well feasible \\
\hline Automation & Bulky/expensive robotics & Well feasible, cost-efficient \\
\hline Single-cell manipulation & Inaccurate, low throughput & Accurate and high-throughput \\
\hline
\end{tabular}

Comparing with conventional macro-scale cell culture, cell densities inside microfluidic devices are largely increased, the nutrients and growth factors deplete more rapidly, and the metabolic wastes build up more easily. (Su, Theberge et al. 2013) As shown in Figure 3.2, the culture condition within microscale dimensions arguably better reflects the physiological environment of cells or cell-populations in vivo than conventional culture. (Mehling and Tay 2014) Cells cultured at macro-scale are kept in a very small surface area to volume ratio. A typical $35 \mathrm{~mm}$ diameter culture dish with $1 \mathrm{~mL}$ of medium has a surface area to volume ratio of $11 \mathrm{~cm}^{-1}$. For microchannel devices, this value is at least 40 times higher. In PDMS mcirochannels (as a gas permeable polymer), a very large surface area to volume ratio is beneficial to cell culture device as the mass transport of gas is very efficient by diffusion. The disadvantage of such large surface area to volume ratio is the increased surface adsorption of proteins. (Walker, Zeringue et al. 2004) In some cases, the high surface area to volume ratio also induces the increased liquid evaporation. Those two affects can be neglected for microfluidic microvessel models as long as there is a perfusion system constantly replenishing nutrients and removing waste product in a timely manner. 


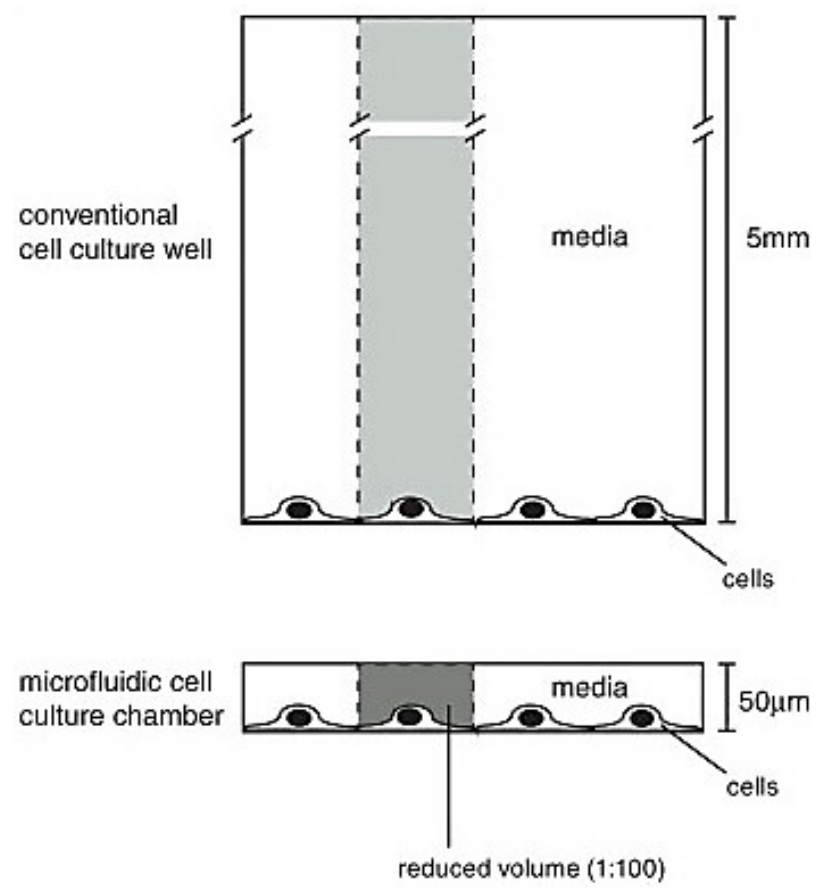

Figure 3.2 Illustration of small-volume effects in microfluidic cell culture devices. Smaller culture media volume results in faster consumption of nutrients and increased concentration of metabolites or secreted molecules, similar to tightly packed tissues. (Mehling and Tay 2014)

For microfluidic long-term cell culture, many techniques have been developed to achieve long-term perfusion. Table 3.2 summarizes the pros and cons of different pumping strategies. (Byun, Abi-Samra et al. 2014) Gravity driven flow is one of the simplest methods to achieve fluid replacement inside microchannels. Only two liquid reservoirs with different heights are needed to start the fluid flow. The flow speed gradually reduces as the height of the liquid level in inlet decreases. Another perfusion flow method is passive pumping via capillary action. When a small drop of fluid is placed in the entrance of a microfluidic channel, it creates more pressure than a bigger droplet at the channel exit, inducing fluid flow. (Walker, Zeringue et al. 2004; Berthier and Beebe 2007) These two passive pumping techniques do not require any complex fluidic interconnections and active pumps. However, it is hard to perform a well-controlled, long-term steady perfusion with those set-ups. The osmosis driven flow is another type of 
passive pumping. When there is a concentration difference across a membrane, and the membrane is only permeable to the solvent but not the solute, it is possible to generate an almost constant and slow flow that can last from hours to days. This perfusion method has been integrated into a microfluidic device by using a cellulose membrane. Deionized water was used as a buffer solution, and poly(ethylene glycol) was used as a driving agent. (Park, Hwang et al. 2007; Park, Kim et al. 2009) However, other research groups, presumably due to the technical difficulty to design such system with the optimized driving agent and solution concentration, have yet to apply this method.

In order to have a greater control of the fluid flow, active pumping methods are often used in microfluidic systems. The most commonly used, commercial available active pumping technique is the syringe pump system. There are large varieties of syringe pumps offered by many different vendors and it is relatively easy to select one that is most suitable for the desired application. A limitation of syringe pump is that the total volume is confined by the size of the syringe, thus sometimes it might be a concern when a large amount of perfusion medium is required over a long period of time. There are other types of active pumps such as vacuum pumps, (Huang, Wang et al. 2013) peristaltic pumps, electrokinetic and electroosmotic pumps. For a typical peristaltic pump, a flexible tube is wrapped around a rotor, and there are rollers that pressing the tube to displace the liquid inside. (Ota and Miki 2011) Due to this mechanism, the flow is pulsatile and oscillatory. The electrokinetic and electroosmotic pumping is induced by an electric field that is parallel to solid-liquid interface, and the flow type is plug-like. It is much easier to integrate electrodes into the microfluidic microchannel than the mechanical actuator. As a result, it is very promising for the design of portable device. However, due to the requirement of a 
relatively high voltage, the heating, bubble formation, and corresponding damage to the cells must be concerned.

Table 3.2 Required characteristics of pumps for cell culture and manipulation. (Byun, Abi-Samra et al. 2014)

\begin{tabular}{|c|c|c|c|c|c|c|c|c|}
\hline Property & Gravity & $\begin{array}{l}\text { Surface } \\
\text { tension }\end{array}$ & Osmosis & Syringe & Vacuum & $\begin{array}{l}\text { Peristaltic } \\
\text { (general, } \\
\text { pneumatic, } \\
\text { Braille pins) }\end{array}$ & $\begin{array}{l}\text { Electric } \\
\text { (electrokinetic, } \\
\text { electroosmotic, } \\
\text { EWOD) }\end{array}$ & $\begin{array}{l}\text { Centrifugal } \\
\text { (lab-on-a- } \\
\text { disc) }\end{array}$ \\
\hline Stability against surface fouling & ++ & + & ++ & ++ & ++ & ++ & - & ++ \\
\hline $\begin{array}{l}\text { Mechanical gentleness (minimal } \\
\text { cell squishing, not too much } \\
\text { shear) }\end{array}$ & ++ & ++ & ++ & + & + & + & ++ & + \\
\hline $\begin{array}{l}\text { Chemical gentleness (minimal } \\
\text { surfactants/toxic chemicals) }\end{array}$ & ++ & ++ & + & ++ & ++ & ++ & + & ++ \\
\hline $\begin{array}{l}\text { Electrical gentleness (no } \\
\text { electrical cell damage) }\end{array}$ & ++ & ++ & ++ & ++ & ++ & ++ & - & ++ \\
\hline Optical accessibility & ++ & ++ & ++ & ++ & ++ & ++ & ++ & ++ \\
\hline $\begin{array}{l}\text { Recirculation of cell culture } \\
\text { media }\end{array}$ & + & - & - & - & - & ++ & + & - \\
\hline Pulse flow generation & - & - & - & ++ & - & ++ & ++ & + \\
\hline Steady flow generation & + & - & ++ & ++ & + & + & + & + \\
\hline
\end{tabular}

\subsection{Long term perfusion system developed for microvessel model}

For our microvessel model, the perfusion rate was calculated based on the desired shear stress distribution (which was discussed in Chapter 6.2, from $1.0 \mathrm{dyne} / \mathrm{cm}^{2}$ to $10 \mathrm{dyne} / \mathrm{cm}^{2}$ ): 0.35 $\mu 1 / \mathrm{min}$ to $4.05 \mu 1 / \mathrm{min}$. The required cell culture/testing period was from 4 days up to two weeks. Passive pumps can induce a flow rate that can reach up to $4 \mu \mathrm{l} / \mathrm{min}$, but the duration of a stable flow at such flow rate is limited (from minutes to couple hours at most). (Meyvantsson, Warrick et al. 2008) 


\subsubsection{Horizontally oriented mini-reservoirs}

As the passive pumping methods cannot achieve the desired flow rate for a prolonged period of time, two alternative strategies were applied to achieve long term perfusion for our microfluidic devices: the horizontally oriented tubes and the syringe pump system. The horizontally oriented tubes were assembled by introducing a hydraulic pressure by placing two identical glass tubes horizontally with a slightly different height as shown in Figure 3.3a-b. (Zhu, Chu et al. 2004) The flow induced by the horizontally oriented tubes was a constant flow, as the height difference did not change, only the lateral positions of the liquid within the tube.
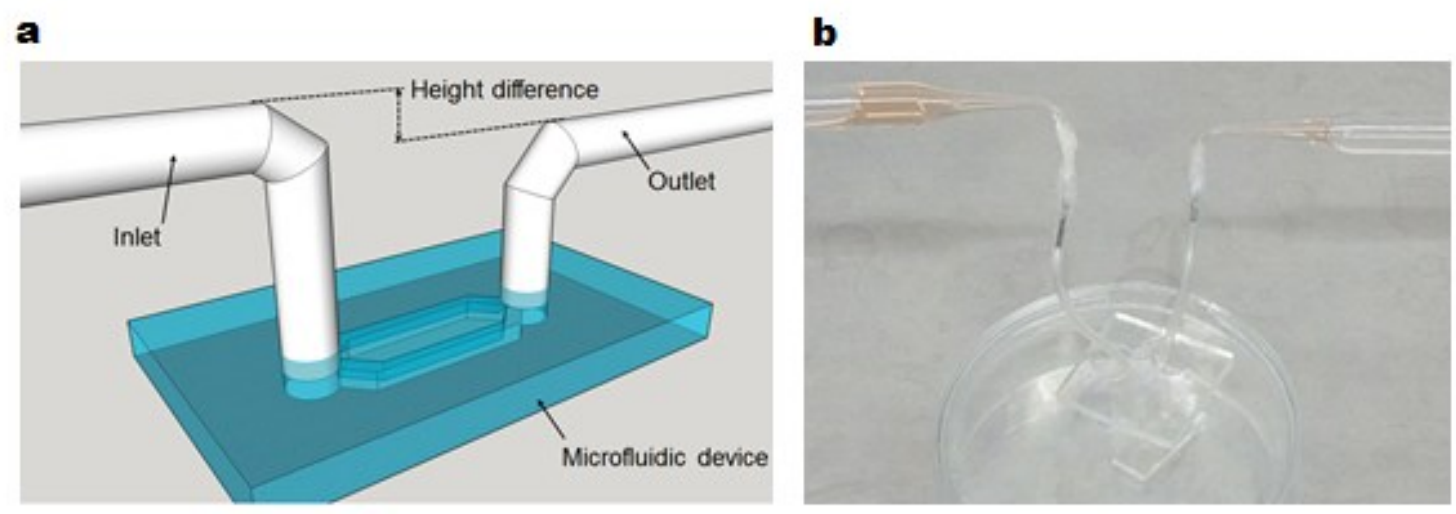

Figure 3.3 a) The hydraulic pressure caused by height difference between the horizontally oriented mini-reservoirs becomes the driven force of the perfusion flow. b) The actual image of the horizontally oriented tubes perfusion set up.

The flow speed measurement was quantified by introducing fluorescent beads $(9 \mu \mathrm{m}$, diluted in culture medium) into the microchannel network devices. The beads worked as tracer particles for flow visualization. The flow velocity of fluorescent beads within the smallest daughter channel was recorded by an inverted microscope. The height difference between the horizontal oriented mini-reservoirs was set by using standard glass slides (1mm thickness). As shown in Figure 3.4, the measured average flow speed increased linearly as the height difference increase. 
The data was presented as the mean \pm standard error (SE) and each individual experiment was performed three times $(n=3)$.

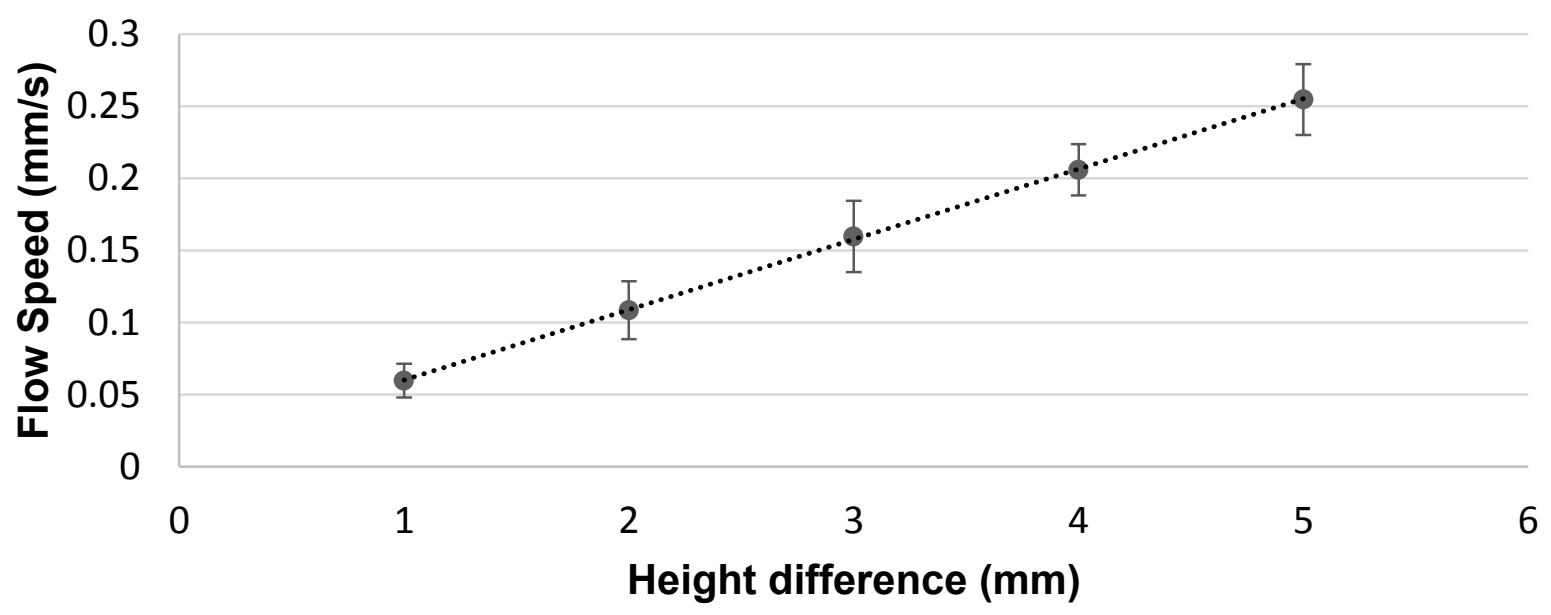

Figure 3.4 Average flow speed $(\mathrm{mm} / \mathrm{s})$ measured in the smallest daughter channel.

\subsubsection{Syringe pump system}

The perfusion provided by horizontally oriented tubes was able to meet the low flow rate required for long-term culture. The total volume inside the tube was $1.5 \mathrm{~mL}$, which can maintain around 48 hours of perfusion. When the tubes were horizontally oriented, the inner diameter of tubes was small enough to prevent the spill-out of the liquid. However, for higher shear stress test, at least $5 \mathrm{~mL}$ of medium was required for a 24-hour test. As a result, it is not possible to introduce desired higher flow rates for shear stress test with this set-up.

A second perfusion method was developed by using a syringe pump system to provide a constant laminar flow for the microfluidic microchannel networks. This kind of pumping system cost more than horizontally oriented tubes, but it is able to provide an accurate yet steady flow field and programmable to apply different flow patterns. For example, it is able to apply the transition from low shear stress to high shear stress with a step function. The gradually increased 
shear stress prevented endothelial cells being peeled off from the substrate or flushed away, which have been observed when the endothelial monolayer was exposed to the sudden increase of wall shear stress.

The PHD ULTRA ${ }^{\mathrm{TM}}$ advanced syringe pump was able to achieve a long-term cell culture between 4 days and two weeks as well as desired shear stresses test, as shown in Figure 3.5. The syringe pump system was able to perfuse 10 syringes at the same time. For long-term culture in this work, $1 \mathrm{~mL}$ syringe was used to perfuse medium into the microfluidic devices; for high shear stress test, $10 \mathrm{~mL}$ syringe was used. The replacement of a new syringe with fresh medium was performed every two days.

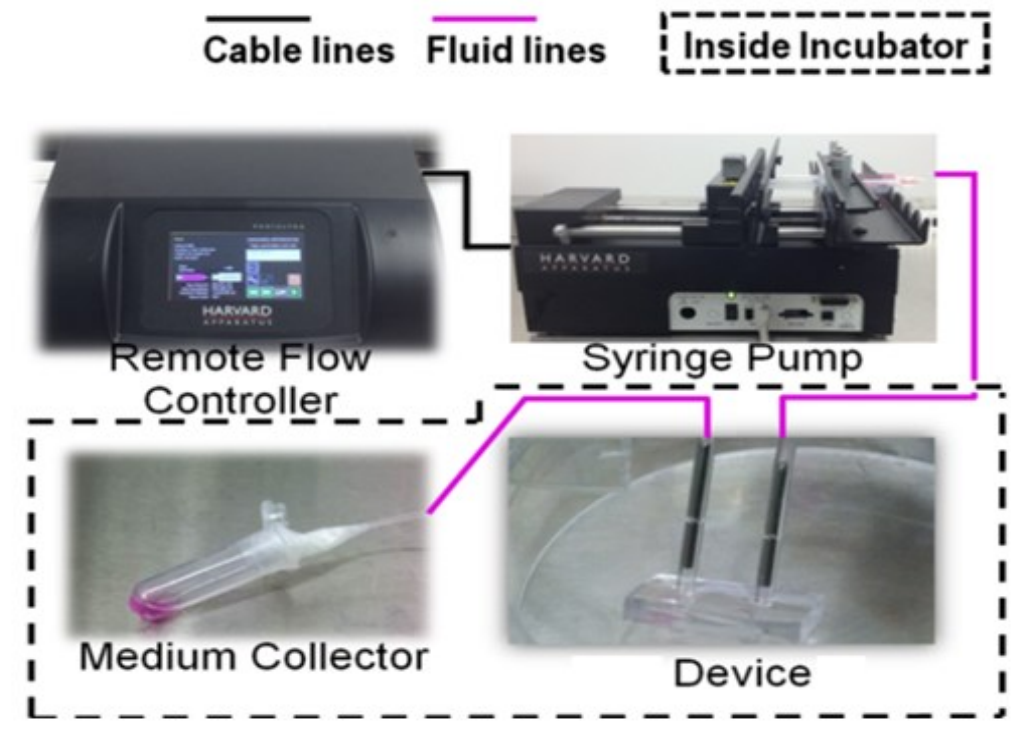

Figure 3.5 Schematic diagram showing the long-term perfusion through the microvessel model using a PHD Ultra syringe pump.

\subsection{Chapter Summary}

Even though it is inheritably possible to apply laminar flow within the microfluidic devices, it is still challenging to apply a well-controlled, stable, and constant perfusion that lasts up to 
couple of days for the endothelial cell culture. In order to achieve the cell viability, proliferation, and functionality that are more close the in vivo microvessels, two perfusion methods were developed to constantly replenish nutrients and remove waste product from cultured cells in a timely manner. Based on the flow rates required to apply physiologically relevant shear stresses, a syringe pump system was used to conduct long-term perfusion of the in vitro microvessel model, as well as dynamic control during the shear stress testing. The perfusion was performed from 4 days up to 2 weeks by using a PHD ULTRA ${ }^{\mathrm{TM}}$ advanced syringe pump system. 


\section{Chapter 4 Standard Fabrication Method for Microfluidic Microchannel}

\section{Networks}

With advanced microfabrication techniques, the flexibility to create patterns and scale sizes provides users with arbitrary design options to mimic the geometries of in vivo microvasculature that allows the functional studies involved in complex flow patterns similar to those found at vessel bifurcations and in regions of high curvature that occur in vivo. In this chapter, the microchannel networks were fabricated with standard photolithography combining with soft lithography technique. This microfluidic microvessel network with thin PDMS film layer bonded on a thin glass substrate (average total thickness is $170 \mu \mathrm{m}$ ) allows excellent light transmission and easy adaptation to both bright field and fluorescence microscopy when using short working distance large numerical aperture objectives. Our study demonstrated that this device is capable for real-time and high-resolution imaging to detect changes in intracellular molecules and cell morphology using fluorescent markers, which is a necessary feature for biological studies.

\subsection{Background}

The development of microfluidic devices has been embraced by engineers over two decades. However, the adaptation and application of microfluidics in mainstream biology is still lacking. According to the recent summary, the majority publications of microfluidics are still in engineering journals (85\%). (Sackmann, Fulton et al. 2014) The equivalent or improved performance of microfluidic devices is not convincing enough for many biologists to switch from current state-of-the-art techniques such as transwell assay and macroscale culture dish/glass slide. (Berthier, Young et al. 2012; Sackmann, Fulton et al. 2014) The most common way to replicate certain features of in vivo shear stress by biologist includes parallel flow chamber and 
cone and plate viscometer. (Chiu and Chien 2011) Microfluidics has the advantage to provide a solution that has not been addressed by those macroscale approaches, such as developing a more physiologically relevant in vitro model. In this chapter, we developed in vitro functional microvessels that could provide a platform for the study of complex vascular phenomena. The microfabrication technique accurately controls the dimensions of the developed microchannels in microscales which mimicking those of in vivo microvessels. In addition, continuous microfluidic perfusion was able to control the mass transfer and flow shear stresses precisely. A confluent endothelial monolayer was grown and fully covered the inside of the entire microchannel network.

\subsection{Device design}

The microchannel networks were designed to mimic the actual sizes of microvessels in vivo. The network design with the smallest channel diameter set as $100 \mu \mathrm{m}$. Figure 4.1 shows the schematic design where the three-level branching microchannels were connected to a single inlet and an outlet.

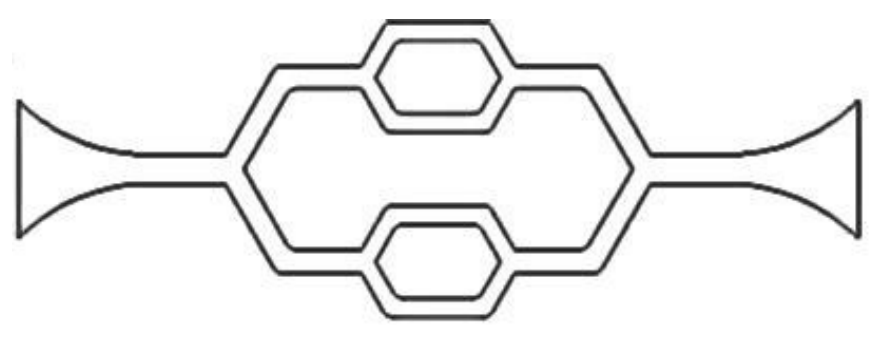

Figure 4.1 The schematic designs of the microchannel networks from the CAD file.

To analyze the spatiotemporal variations of the fluid flows and pressure distributions inside the microchannel networks, a fluid dynamic analysis model using COMSOL multiphysics software (Version 4.0.0.982, COMSOL Inc.) was built to numerically simulate shear flows and pressure distributions. (Huang, Li et al. 2012) Incompressible Navier-Stokes equations were 
chosen as governing equations for the fluids, and no-slip wall boundary condition was set along the internal surface of the microchannels except for the inlet and the outlet.

\subsection{Device fabrication}

A PDMS microchannel device was fabricated by photolithography and soft lithography process (McDonald and Whitesides 2002) as shown in Figure 4.2. SU-8 photoresist (SU8-2050, Microchem) was spin-coated on a pre-cleaned silicon wafer with the thickness of $100 \mu \mathrm{m}$. The wafer was rinsed with acetone and methanol for 5 minutes each time. It was then baked on hot plate $\left(150^{\circ} \mathrm{C}\right)$ over 30 minutes for dehydration. After transfer designed pattern from a film mask with the exposure of UV light, the silicon wafer was developed and used as a master mold. (Figure 4.2a-c) PDMS (Sylgard 184, Dow 103 Corning Corporation) was mixed with the wt. ratio of 10:1, and casted onto the master mold to replicate the microchannel patterns. The PDMS was released after baked $60^{\circ} \mathrm{C}$ in the oven over 3 hours. (Figure $4.2 \mathrm{~d}$ ) The inlets and outlets for the cell loading and tubing connections were created with a puncher. In a typical confocal microscopy system, the objective lenses with high numerical apertures (NA) have a limited working distance of couple hundred microns. (Pawley 2010) Therefore, in order to integrate the microfluidic devices to such system, glass coverslip is chose to be the substrate of the microchannel. The channel side of the PDMS and the coverslip were treated with oxygen plasma for 30 seconds then permanently bonded together. (Figure 4.2e) 
a

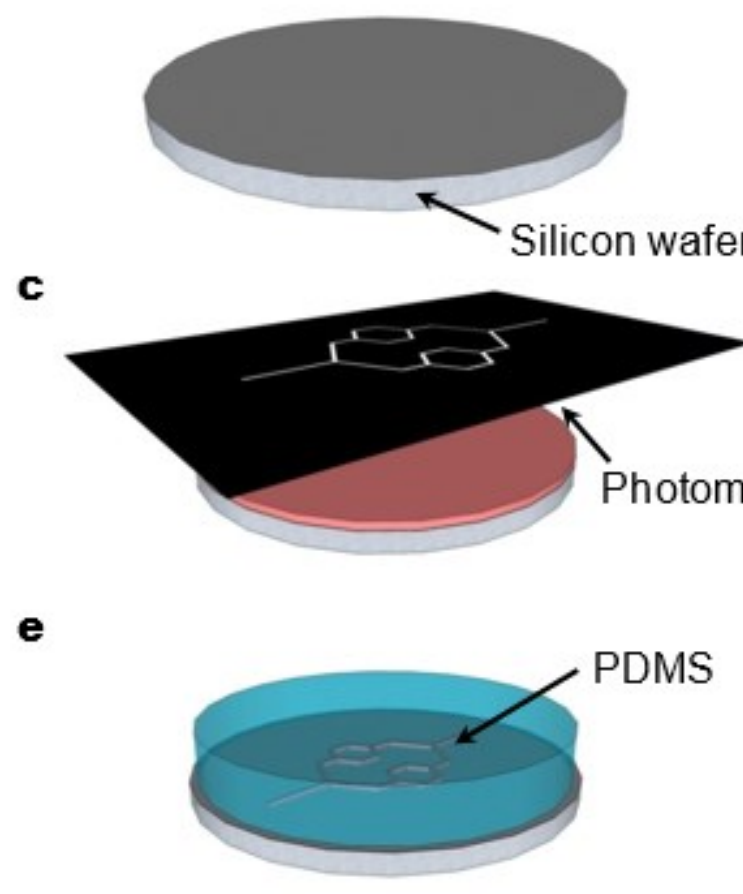

b

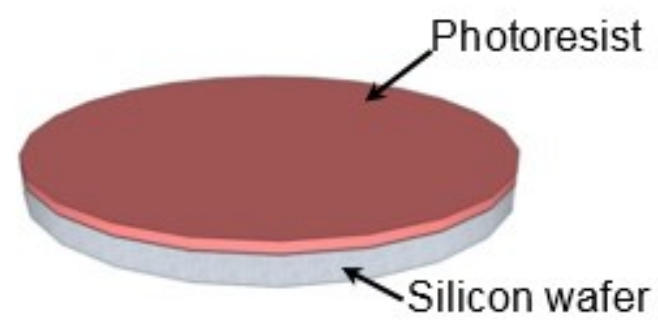

d

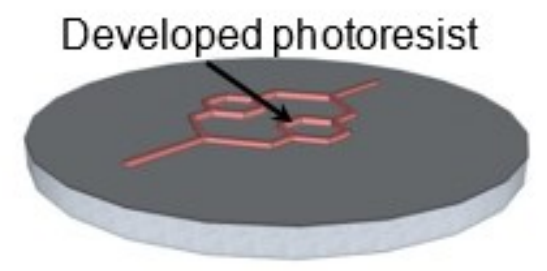

$\mathbf{f}$

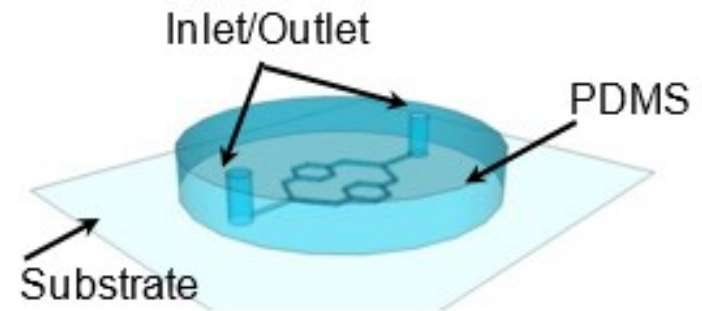

Figure 4.2 The schematic fabrication procedures for PDMS devices. a-b) SU-8 photoresist was spin-coated onto the pre-cleaned silicon wafer. c) The photoresist was exposed to UV light though the photomask. d) Developed flow chamber/microchannel pattern as the master mold. e) PDMS solution was casted onto the master mold and cured. f) The flow chamber/PDMS microchannel structure was bonded onto the substrate.

The bare glass substrate and the PDMS surfaces are not ideal for cell attachment. To promote cell adhesion and maintain cell morphology, it is necessary to introduce surface modifications. Most commonly used coatings include fibronectin, collagen, laminin, matrigel, IPN, and polydpoamine. (Collier, Anderson et al. 2004; Heino 2007; Lee, Dellatore et al. 2007; Pi, Ding et al. 2008; Ku, Ryu et al. 2010) The fibronectin coating (100 $\mu \mathrm{g} / \mathrm{ml})$ was used in this work. 


\subsection{Endothelial cell seeding and long-term perfusion}

\subsubsection{Endothelial cells seeding and culture method}

Primary human umbilical vein endothelial cells (HUVEC, Lonza) were cultured in MCDB 131 culture medium (Gibco) supplemented with 10\% fetal bovine serum (FBS, ATCC) in tissue cultured flasks, pre-coated with $0.2 \%$ gelatin. The cells between the second and fifth passage were used for the experiment.

When the HUVECs approached to confluence, the cells were first rinsed with HEPES Buffered Saline Solution, and then treated with Trypsin/EDTA for 2 minutes at $37^{\circ} \mathrm{C}$. After cells were detached from the substrate, the trypsin/EDTA was neutralized with trypsin neutralizing solution. The detached cells were then transferred to a sterile centrifuge tube and centrifuged at $220 \times \mathrm{g}$ for 5 minutes to pellet the cells. The HUVECs were prepared at a concentration of $2 \times 10^{6}$ $\sim 4 \times 10^{6}$ cells $/ \mathrm{mL}$ in culture media supplemented with $8 \%$ dextran $(70 \mathrm{kDa}$; Sigma) for seeding. Dextran increases the viscosity of the media to enable fine control over seeding density of endothelial cells.

Plasma-assisted oxidation of the PDMS microchannels introduces silanol groups $(\mathrm{SiOH})$ on the surfaces that renders the surface hydrophilic and aids in functionalization. Then the device was loaded with DI water and exposed to UV light for 8 hours in a laminar biosafety hood. After UV sterilization the device was rinsed with $1 \times$ phosphate buffered saline (PBS), coated with fibronectin $\left(100 \mathrm{ug} / \mathrm{ml}\right.$, diluted with PBS), and incubated in the refrigerator $\left(4^{\circ} \mathrm{C}\right)$ overnight. Different extracellular matrix (ECM) proteins (fibronectin, gelatin, and collagen) were tested for cell attachment, and the best result was found to be fibronectin coating. Before the cell seeding, the device was rinsed with PBS again, loaded with culture medium and incubated over 15 
minutes at $37^{\circ} \mathrm{C}$. A $10 \mu \mathrm{L}$ droplet of HUVEC cells was placed at the inlet, and a slow flow was created by either tilting the device, or placing a glass pasteur pipets (the inner diameter of the pipets is around $1.5 \mathrm{~mm}, \mathrm{VWR}$ ) at the outlet (no vacuum is needed) to introduce a capillary flow. The bright field image of the initial loading is shown in Figure 4.3a, where the cells were starting to attach onto the microchannel.

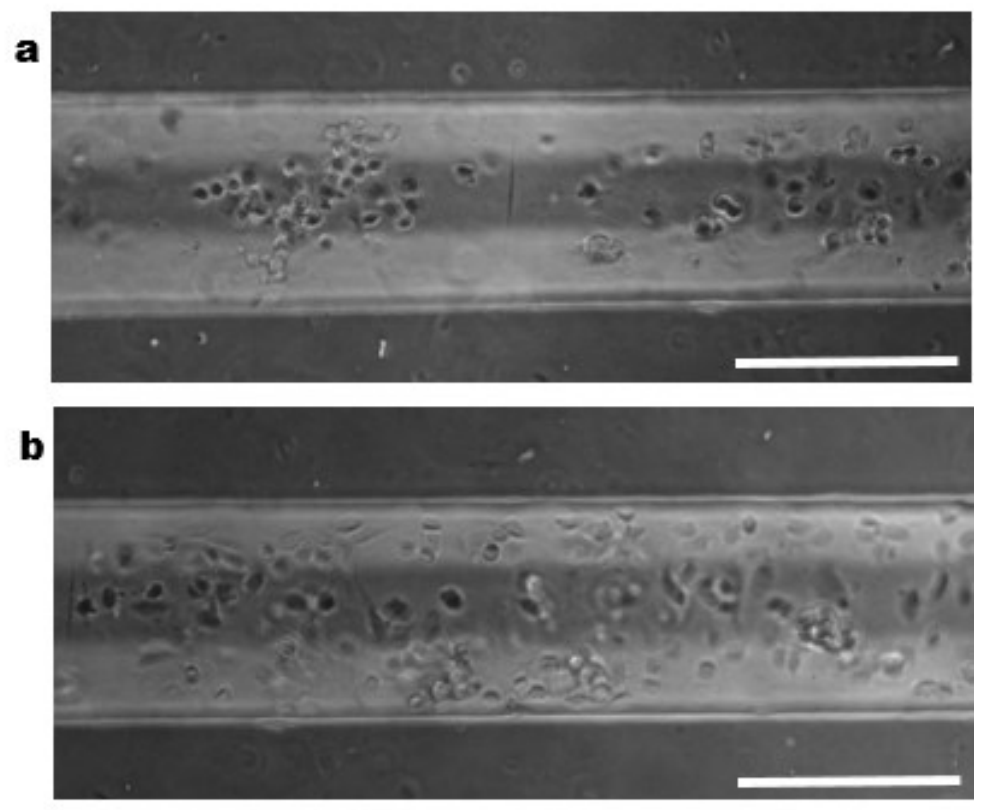

Figure 4.3 a) Bright field image of the HUVEC initial loading. b) Bright field image of the HUVEC attachment 15min after the initial loading. Scale bars: $100 \mu \mathrm{m}$.

The key for a successful cell loading is to keep a low flow velocity, otherwise most of the cells would not attach uniformly inside the microchannel. After 15-20 minutes, attached cells were visually examined under the microscope and additional loading can be performed if necessary as shown in Figure 4.3b. After the device reached a satisfying seeding density, it was washed with fresh culture medium to remove the extra dextran. After 5-6 hours of static culture, HUVEC cells were fully attached and spreading out. 


\subsubsection{Long-term cell culture under constant perfusion}

With the initial cell loading concentrations of $2 \sim 4 \times 10^{6}$ cells/mL, confluent monolayers developed within 3-4 days under a constant flow of culture media. Figure 4.3 shows the confocal images of endothelial cell with F-actin and cell nuclei staining at different regions of the network in the same device. The results illustrated that HUVECs successfully cultured the entire inner surface of the network to confluence.

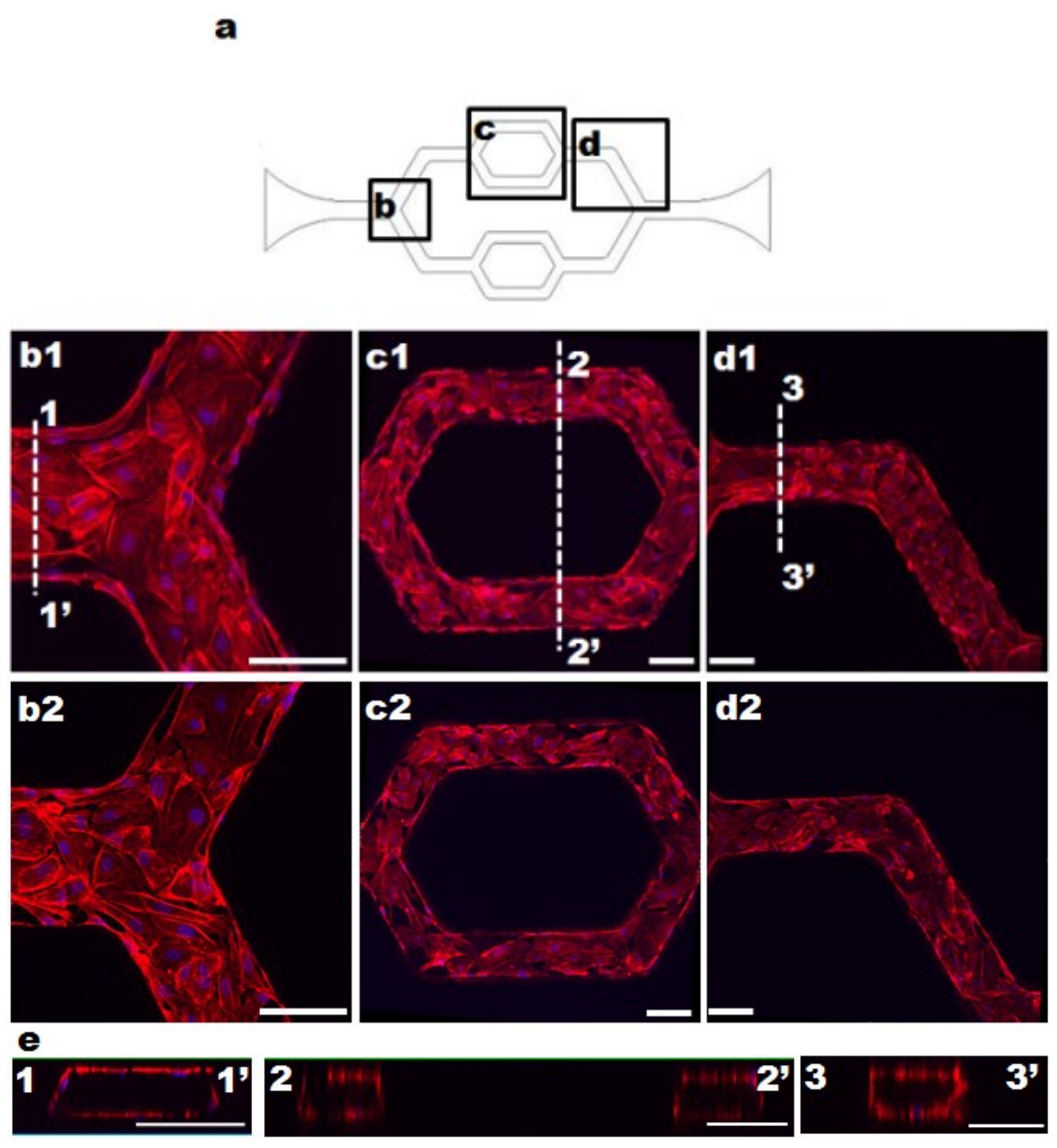

Figure 4.3 The representative confocal images show the HUVECs successfully cultured throughout the inner surfaces of the entire microchannel network. a) The schematic image of the network with selected regions as shown in b-d. b-d) HUVECs were stained with F-actin and cell nuclei in each region, where b1, c1 and d1 show the top surfaces of the channel, respectively. b2, c2 and $\mathrm{d} 2$ show the bottom surfaces of the channel, respectively. e) The cross-sectional images at each region. The locations of the cross-sections were indicated as 1-1', 2-2', and 3-3' in b1-d1, respectively. Scale bars: $100 \mu \mathrm{m}$. 


\subsection{Chapter summary}

The main difference of using this microchannel network compared with traditional cell culture is that the cells, instead of growing under static conditions at 2-dimensional flat surface, were growing in a three dimensional (3D) channel with continuous flow, which made the cell culture environment closer to the in vivo situation.

By using computational fluid dynamic simulation method, a numerical 3D model was built. It was able to provide an accurate estimation of the wall shear stress distribution. Based on the simulation result, the shear stress that was comparable to the in vivo venous system (1 to 10 dyne $/ \mathrm{cm}^{2}$ ) was applied to the microvessel model by using syringe pump perfusion. (Lipowsky 1995; Malek, Alper et al. 1999) The details for shear stress simulation was discussed in Chapter 6.

In order to integrate our microvessel model into microscopic systems, the PDMS channel was bond onto a thin coverslip substrate for real time high-resolution imaging. This standard microfabrication process was more convenient to achieve the desired substrate thickness for confocal microscopy. This fabrication method resulted in rectangular shaped microchannels, as shown in Figure 4.3. The cells attached to the corners did not exhibit abnormal morphology and reached confluence inside the microchannel networks after a few days of perfusion.

This model was able to perform quantitative analysis of the key features of the developed microvessels. The characterization of the endothelial adherence junction staining, cytoskeleton reconstruction under different shear flow, and $\left[\mathrm{Ca}^{2+}\right]_{\mathrm{i}}$ and NO response to ATP were discussed in Chapter 6 and 7. 


\section{Chapter 5 Multi-Diameter Microfluidic Microchannels}

Current methods for formation of microvascular channels are limited with non-circular channel cross-sections, complicated fabrication, and less flexibility in microchannel network design. To address current limitations in creating of engineered microvascular channels with complex three-dimensional (3-D) geometries in the shape of microvessels, we developed a reproducible, inexpensive, and highly accurate micromanufacturing process combined with photolithographic reflowable photoresist and soft lithography techniques to fabricate cylindrical microchannel and networks. A positive reflowable photoresist AZ P4620 was used to fabricate a master microchannel mold with semi-circular cross-sections. By alignment and bonding together two polydimethylsiloxane (PDMS) microchannels replicated from the master mold, a cylindrical microchannel or multi-diameter microchannel network was created. In addition, primary human umbilical vein endothelial cells (HUVECs) seeded inside the chip showed that the cells successfully lined the inner surface of the microchannels under controlled perfusion lasting for a time period between 4 days to 2 weeks and cultured to confluence. The fabricated cylindrical microchannels and network can provide platforms for investigation of microvascular cells growing inside of cylindrical channels under shear flows and lumen pressures.

\subsection{Background}

The creation of engineered microvascular channels with complex three-dimensional (3-D) geometries in the shape of microvessels presents a major challenge to the field of microvascular research and tissue engineering. There have been reports for making microvascular channels using conventional lithography methods, (Kaihara, Borenstein et al. 2000; Shevkoplyas, Gifford

et al. 2003; Lu, Koo et al. 2004; Emerson, Cieslicki et al. 2006) but all of such devices had employed rectangular, square or trapezoidal cross-sections of channels. Such channels impose 
widely varying fluid shear stresses and non-physiological geometries on cells in different channel positions (Zeng, Lee et al. 2006) that can induce significant variations in cell physiology. (Nerem, Alexander et al. 1998; Fisher, Chien et al. 2001) It is well accepted that the degree of shear stress imposed on luminal endothelial cells, which depends strongly on the geometry of the flow channel (Camp, Stokol et al. 2008) affects their differentiation state, (Riha, Lin et al. 2005) alignment and elongation, (Kadohama, Akasaka et al. 2006) barrier function formation, (Colgan, Ferguson et al. 2007) gene expression, (Warabi, Wada et al. 2004; McCann, Peterson et al. 2005) and response to inflammatory stimuli. (Oblak, Root et al. 2006) For these reasons, the geometry of the channel should replicate as closely as possible to the geometry of in vivo microvessels because of its essential role in the formation and maturation of vessels.

Microfabrication techniques can precisely control the fabricated structures dimensions in the range from micrometers to millimeters. They have been investigated for fabrication of microchannels to close approximate the geometry of in vivo microvessels, which adopt a roughly circular cross-section with radii between 30 and $300 \mu \mathrm{m}$. (Young, Woodford et al. 2013) Typical substrates for fabricating microchannels are silicon (de Boer, Tjerkstra et al. 2000; Liu, Stremler et al. 2000; Agarwal, Ranganathan et al. 2008), glass (Rodriguez, Spicar-Mihalic et al. 2003), and polymers (Borenstein, Tupper et al. 2010). The efforts to create relatively well-controlled circular cross-section of channels were reported by wet chemical etching (Seo, Bae et al. 2004), reactive gases such as SF6, CF4 and XeF2 (Camp, Stokol et al. 2008), ionized gas dry etching (Chin, Yu et al. 2011), femtosecond laser writing followed by chemical etching (Maselli, Osellame et al. 2006; Crespi, Gu et al. 2010), electroplating combined with hot embossing and thermal bonding (Borenstein, Tupper et al. 2010), thin film coating to modify the channel geometries and embedded sacrificial elements (Abate, Lee et al. 2008; Bellan, Singh et al. 2009; 
Song, Lee et al. 2010). However, these fabrication methods involved toxic gases, can only achieve a limited maximum channel length (Abgrall and Gue 2007), less flexibilities for complicated channel designs, and the fabricated channels were not suitable for long-term cell growing. These shortcomings limited their applications with microvascular cells and their research.

Since the inception of soft lithography (Duffy, McDonald et al. 1998; Xia and Whitesides 1998), polydimethylsiloxane (PDMS) based microfluidic channels have been widely used for biological and chemical applications. The fabrications of circular cross-section of microchannels in PDMS were demonstrated by various molding techniques, which either directly molded the circular channels inside of PDMS or by PDMS replications from rounded master molds. Metal wires (Song, Lee et al. 2010), stain steel needles (Chrobak, Potter et al. 2006), and liquid PDMS re-modified channels (Fiddes, Raz et al. 2010), and pressurized air streams (Abdelgawad, Wu et al. 2011) have been reported to directly create a single straight cylindrical channel in PDMS. These methods have limitations for fabricating channel networks, well-controlled channel dimensions, and the geometries of the fabricated channels could be varied from experiments to experiments. The fabrications of rounded master molds were demonstrated by photoresist melting (Wang, Ho et al. 2007; Chen, Chen et al. 2010), isotropic wet etching of the substrate molds (Shao, Wu et al. 2009), mechanical micromilling (BurakáOzdoganlar 2011). These methods provide the flexibility for the channel designs, and the fabricated molds can be re-used for PDMS replications. However, the reported photoresist melting method created a bow shape cross-section. One concern about the bow shaped channel is the difficulty for cells seeding and growing in certain areas of the molded microchannels. Mechanical micromilling shows lack of 
precisely control channel dimensions, which may cause relative larger variations and surface roughness in the small scales.

In this chapter, we presented a convenient, inexpensive, reproducible, and highly accurate microfabrication approach to create both a single cylindrical microchannel and a circular crosssection of microchannel network in PDMS by the combination of photolithographic photoresist reflow techniques and soft lithography. The geometries of the microchannels closely mimic the dimensions of microvessels in vivo. The microchannels in PDMS can be used as a scaffold for growing microvascular cells inside under shear flows and lumen pressures, and further be used as a mold for developing more physiological relevant tissue scaffolds for morphogenesis and tubulogenesis.

\subsection{Design and fabrication}

\subsubsection{Microchannel design}

Conventional photolithography, in which light is projected through a patterned mask onto a photosensitive substrate, generally creates rectangular or square cross-section of patterned features (Whitesides, Ostuni et al. 2001; Becker and Gärtner 2008; Leong, Zarafshar et al. 2010), which is not preferred for microvascular cell attachment as previous discussed. The photoresist reflow technique eliminates this limitation and is able to create a semi-circular cross-section of pattern. It involves melting photoresist structures to form curvature channels shaped by the surface tension of the liquid resist. However, the profiles of reflowed photoresist can be much more complex and resulted from certain parameters, such as polymeric nature of the photoresist, reflow temperature, resist boundary movement during reflowing, and outgassing. ( $\mathrm{O}^{\prime} \mathrm{Neill}$ and Sheridan 2002) PDMS replication using the reflow resist mold will create microchannels with 
semi-circular cross-sections. A circular cross-section of a channel can be created by aligning and bonding of two identical PDMS molds together.

As shown in Figure 5.1, a single straight microchannel and a microchannel network were designed to mimic the actual sizes of microvessels in vivo. We designed the single channel with different diameters of $25 \mu \mathrm{m}, 50 \mu \mathrm{m}, 100 \mu \mathrm{m}, 150 \mu \mathrm{m}$, and $200 \mu \mathrm{m}$ (Figure 1a).
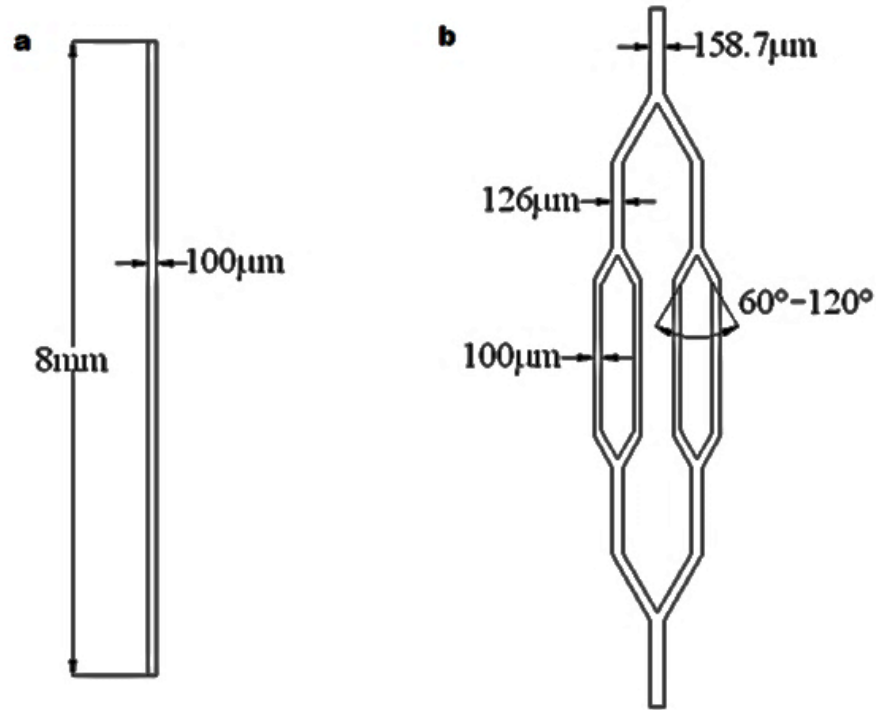

Figure 5.1 Schematic designs of a single microchannel and a microchannel network. a) A straight channel. (Width: $100 \mu \mathrm{m}$ and length: $8 \mathrm{~mm}$ as an example) b) A network with multi-branch channels. As an example, the channel widths at different branch channels were calculated based on the Murray's law.

The design of a multiple branch channel network is based on the Murray's law, (Murray 1926) which states that the cube of the radius of a parent branch equals the sum of the cubes of the radii of the daughters.(Sherman 1981) Figure 5.1 shows an example of the network design with the smallest channel diameter set as $100 \mu \mathrm{m}$, and the diameters of its upper branch channels were calculated based on the Murray's law. The angles at the bifurcations were designed from $60^{\circ}$ to $120^{\circ}$ to mimic the histological conditions so that the channel network design matches the 
microvascular vessel network in vivo for maintaining the fluid flow at a required level. (Murray 1926; Zamir and Medeiros 1982; Gafiychuk and Lubashevsky 2001)

\subsubsection{Device fabrication}

The positive reflow photoresist P4620 (AZ Electronic Materials) works well for patterning features down to $5 \mu \mathrm{m}$, and the film thickness can reach up to $60 \mu \mathrm{m}$ with different spin-coating protocols, which makes it suitable as a mold material for the fabrication of the microvascular channels which require different diameters or aspect ratios. In our fabrication procedures, conventional photolithography followed by resist reflowing was applied for the master mold fabrication. (Figure 5.2) Both the single channel and the network patterns were designed as discussed in the above section, drawn by AutoCAD, and transferred into a photomask.

Silicon wafers were pre-cleaned and hard baked at $150{ }^{\circ} \mathrm{C}$ for $1 \mathrm{~h}$ to enhance the adhesion between the wafers and the AZ P4620. (Figure 5.2a) The thickness of the spun AZ P4620 is limited to $30 \mu \mathrm{m}$. For a microchannel with a larger diameter, we used a double-coating procedure to get a thicker AZ 4620 film. The first layer of AZ P4620 $(10 \sim 30 \mu \mathrm{m})$ was spun onto the substrate and baked at $110{ }^{\circ} \mathrm{C}$ for $90 \mathrm{~s}$, and then, the second layer of AZ P4620 $(10 \sim 30 \mu \mathrm{m})$ was spun on top of the first layer and baked at $110{ }^{\circ} \mathrm{C}$ for 3 minutes. (Figure 5.2b) The photoresist film was UV exposed using a flood exposure system (OAI model 150, Figure 5.2c) and followed by the development in AZ $400 \mathrm{~K}$ developer diluted with deionized (DI) water (1:2 (v/v)) for 4 minutes. (Figure 5.2d) Finally, the patterned AZ P4620 was reflowed and rounded at $120{ }^{\circ} \mathrm{C}$ for 4 minutes to form a half cylindrical channel with a semi-circular cross-section which was used as a master mold for PDMS replication. (Figure 5.2e) 

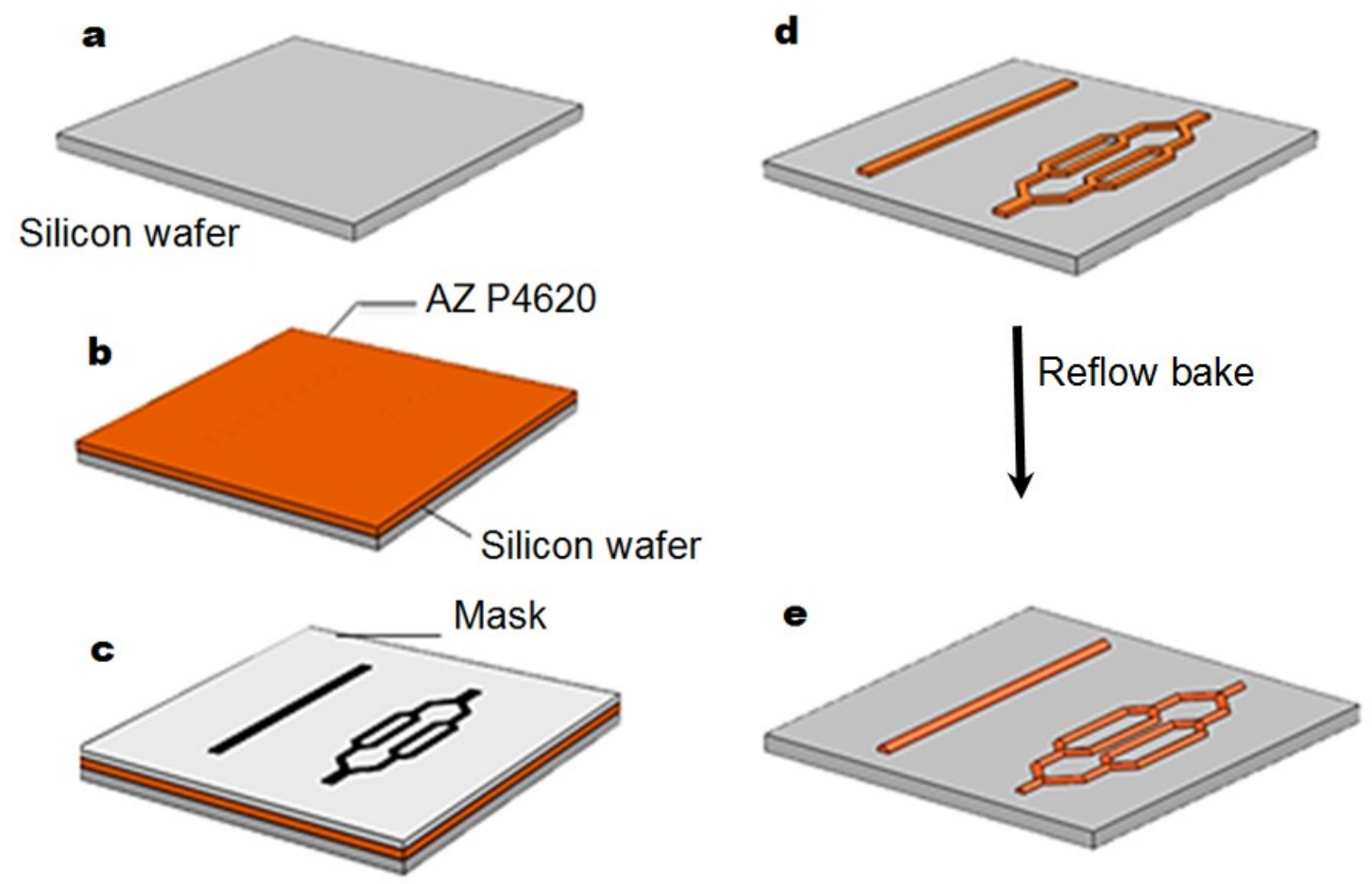

Figure 5.2 The schematic fabrication procedures for AZ P4620 master molds. a) A precleaned silicon substrate. b) Two layers of positive photoresist AZ P4620 were spun onto the silicon substrate, respectively. The second layer of AZ P4620 was applied after the first layer was baked and dried. c) The photoresist was exposed under the UV light through the mask. d) The patterned structure after AZ $400 \mathrm{~K}$ development. e) The reflow photoresist patterns with semi-circular cross-sections after the reflow at $120{ }^{\circ} \mathrm{C}$ for 4 minutes.

PDMS solutions were prepared at the ratio of 10:1 (base : curing agent) and cast onto the AZ P4620 master mold twice, respectively. (Figure 5.3 a-b) The PDMS layers together with the master molds were baked at $60{ }^{\circ} \mathrm{C}$ for $3 \mathrm{~h}$ to cure the PDMS, and then, the PDMS layers were peeled off the molds and treated with 30 s oxygen plasma. They were aligned under an optical microscope and bonded together, which formed a microchannel with a circular cross-section. (Figure $5.3 \mathrm{c}-\mathrm{d})$ 

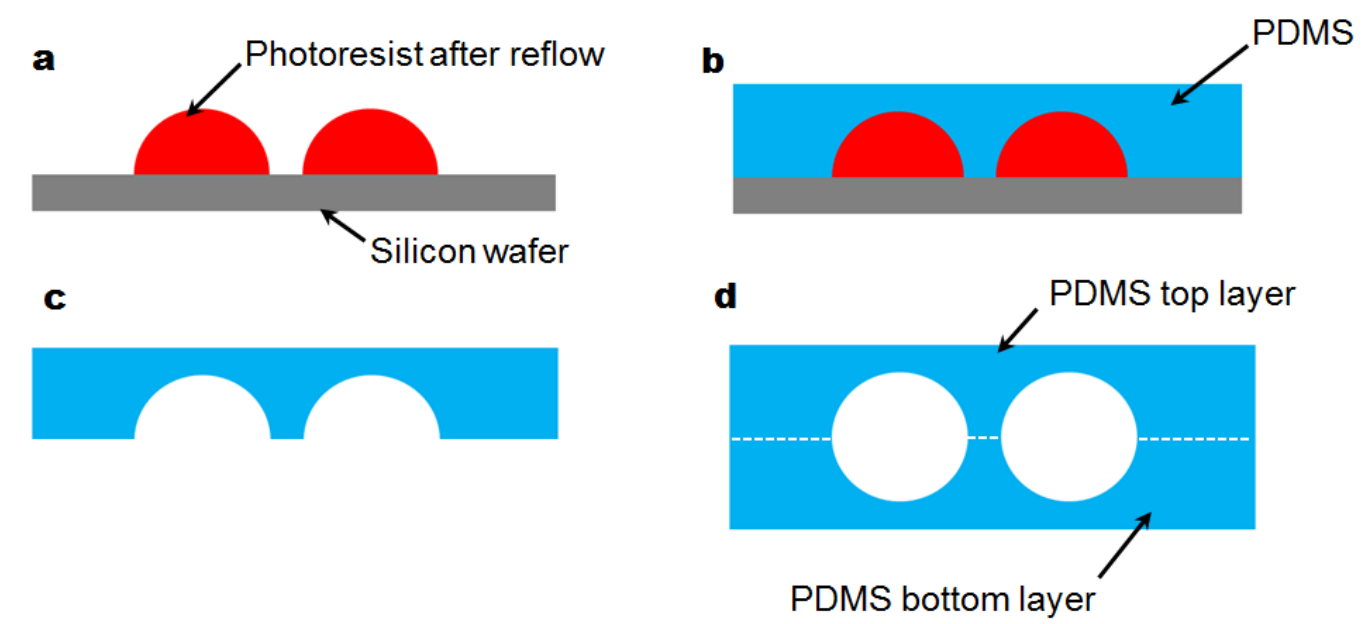

Figure 5.3 The schematic fabrication procedures for cylindrical microchannels in PDMS. a) The reflow AZ P4620 microchannel network. b) Cast PDMS solution onto the AZ P4620 mold. c) The cured PDMS layer. d) Aligned and bonded two PDMS layers to form microchannels with circular cross-sections.

\subsection{Result and discussion}

\subsubsection{AZ P4620 Master mold}

Figure 5.4a and 5.4b show scanning electron microscope (SEM, JEOL JSM-7600F) images of the AZ P4620 photoresist mold before and after the reflow process, respectively. Figure 5.4c and Figure 5.4d show the replicated PDMS channels from the molds before and after reflow, respectively. The cross-section of the PDMS microchannel indicates the profile changes from a rectangular to a semi-circular cross-section.

The reflow process involves the melting of the patterned photoresist, and the liquid resist surfaces are pulled into a shape that minimizes the energy of the system. (Daly, Stevens et al. 1990) A cooling and solidification phase is followed the melting process. In ideal conditions, the shape of the reflowed photoresist will be very close to a semi-circular surface. However, several parameters are involved and make the profiles more complex, such as critical angle at which the 
photoresist meets the solid substrate, material evaporation, temperature gradient, outgassing, resist boundary movement, variation of substrate, and polymeric nature of resists.
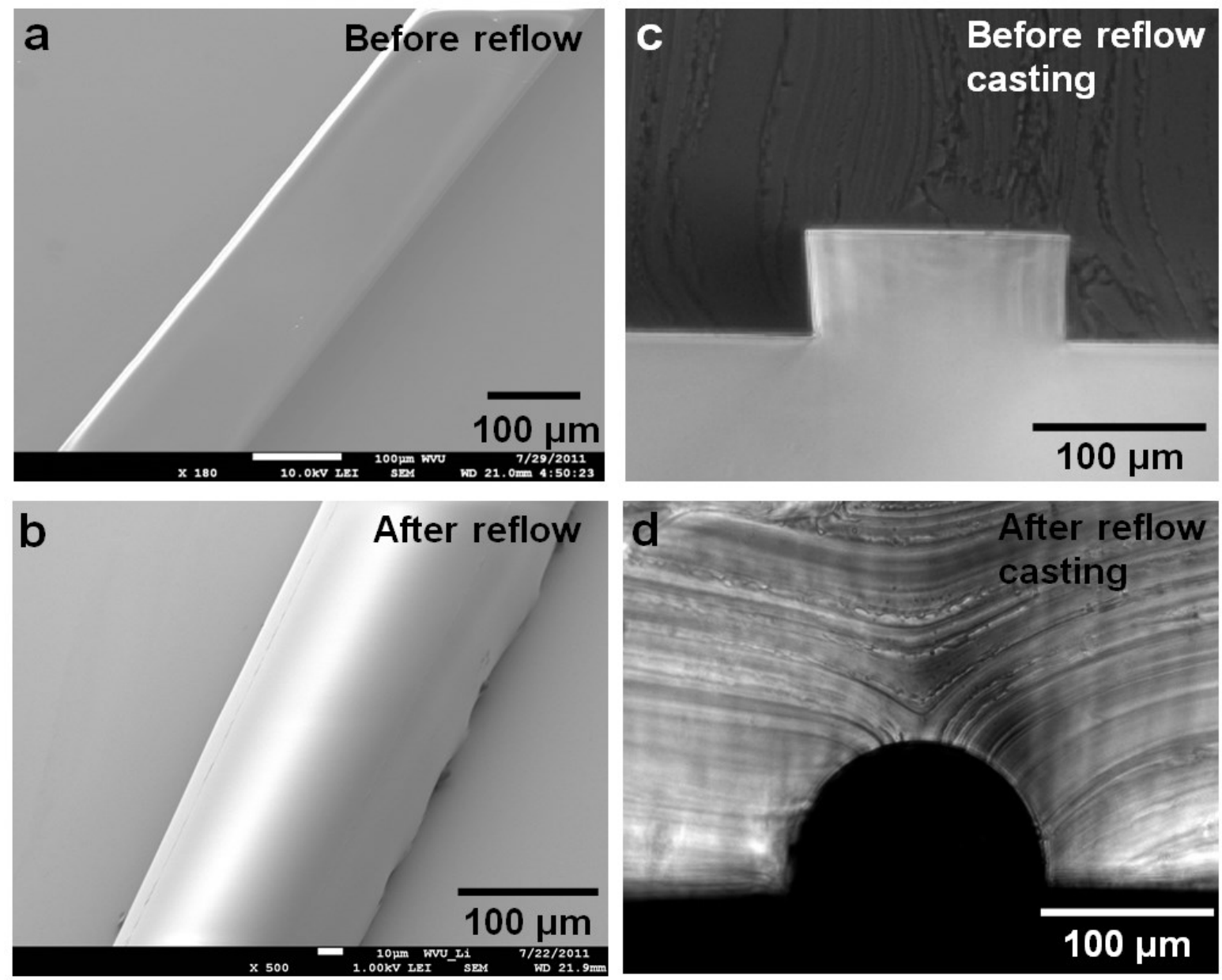

Figure 5.4 SEM shows the developed AZ P4620 photoresist. a) before reflow b) after reflow. c) Molded PDMS showed the cross section of the resist before reflowing; the picture shows a rectangular cross section. d) Molded PDMS showing a semi-circular cross-section of the resist after reflowing. The cross section after reflow was controlled by the initial dimension design of the pattern and reflow temperature.

The magnitude of the critical angle is governed by the surface tension of the liquid resist, the surrounding air, and the substrate properties. (De Gennes 1985) In addition, the temperature and timing during the reflow process can result in a volume change causing the boundary to move 
and hence varying the critical angle. (Elias 1997) Therefore, variations in this critical angle, resulting in different rounding profiles of the reflow resist, will occur if the reflow temperature and timing are varied, the substrate is non-uniform, or the liquid/substrate interface moves. (Sekimoto, Oguma et al. 1987; Voinov 1999)

To get a cylindrical surface for a given width of microchannel, one particular volume of the resist is required. The film thickness required for an ideal cylindrical case can be pre-determined given a required width and focal length of the microchannel as $H=\frac{1}{2 E}\left[\frac{R^{2}}{r} \sin ^{-1}\left(\frac{r}{R}\right)-R+h\right]$ (5.1), where $\mathrm{H}$ is the required spun-film thickness, $\mathrm{r}$ is the half of the channel width, $\mathrm{R}$ is the constant radius of curvature, $\mathrm{h}$ is the central height of the curvature, and $\mathrm{E}$ is the ratio of resist volumes of the microchannel before and after reflowing.

If cylindrical surfaces with different radii are required through a microchannel network, it is necessary to vary the width of the microchannels, resulting in variations in the volumes, at different regions in the network. Deviations from the cylindrical surface could occur when the volume used to produce the microchannel varies from the volume necessary to produce the cylindrical case. (Daly, Stevens et al. 1990)

In addition, an ideal volume for getting a cylindrical surface must make allowance for the effect of material evaporation during the reflow process. (Jay and Stern 1994) To successfully fabricate a single cylindrical channel or a cylindrical channel network with different radii, the resists widths/volumes, reflow temperatures and timing, critical angles, boundary movement, and resist and substrate properties must be considered.

During the fabrication process, it was noticed that small defects appeared on the photoresist surface when the reflow baking temperatures were higher than $120^{\circ} \mathrm{C}$ or longer baking (more 
than 4 minutes) was performed. (Figure 5.5) The fact of these small defects could be possibly caused by outgassing, which can affect the channel profile formation in causing gas bubbles in the resist during reflow baking. The outgassing issue was solved by lowering the baking temperatures.

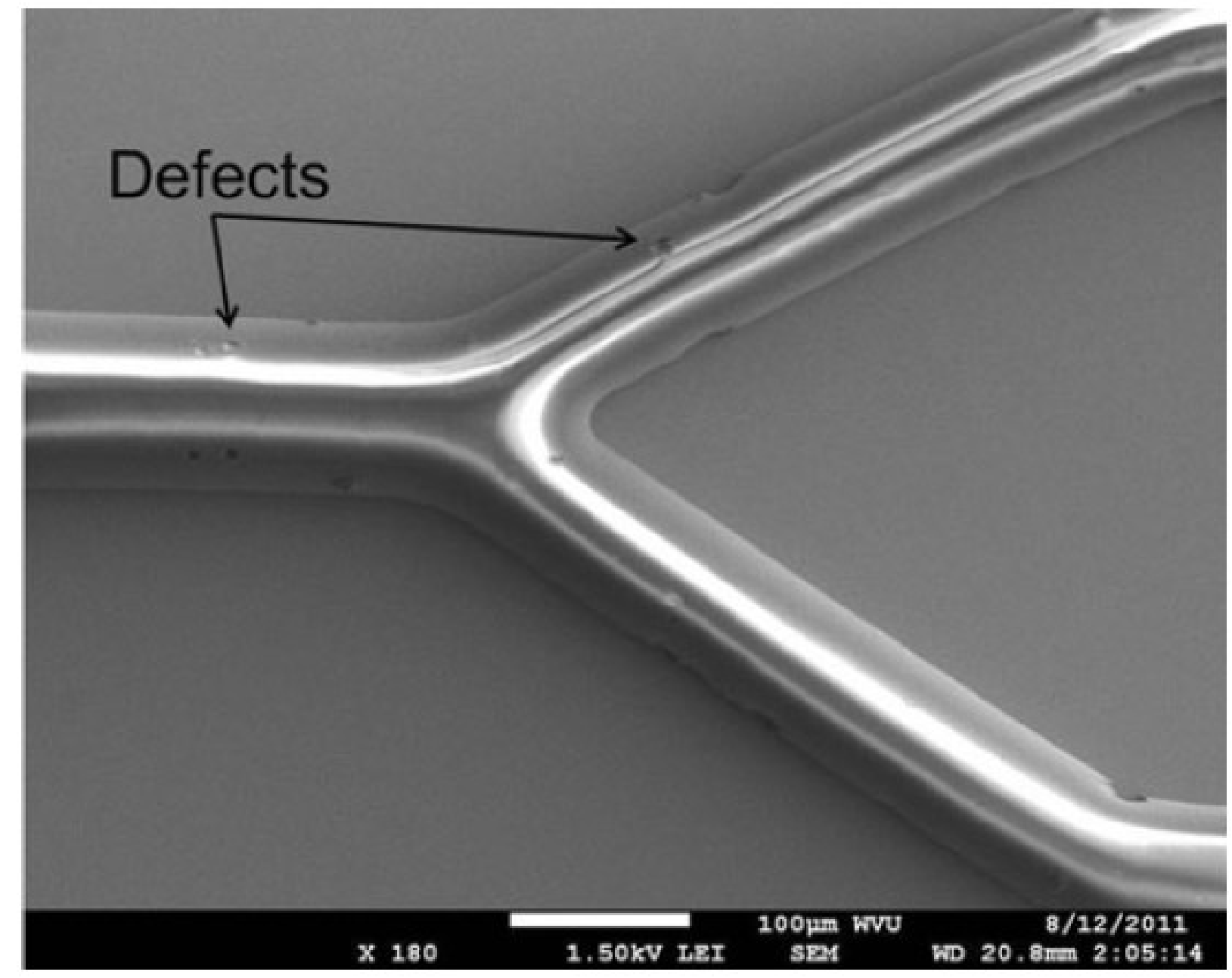

Figure 5.5 SEM shows small defects on the reflowed mold which is caused by high reflow baking temperatures $\left(>120^{\circ} \mathrm{C}\right)$ or long baking time ( $>4$ minutes).

The geometries of in vivo microvessels adopt a roughly circular cross-section with radii between 30 and $300 \mu \mathrm{m}$, thus the fabricated microchannels are required to closely approximate these geometries. According to the protocol of AZ Electronic Materials, a 20-30 $\mu \mathrm{m}$ thick AZ 
P4620 film (before reflow) can be easily obtained by adjusting the coating speeds in the range lower than $1000 \mathrm{rpm}$. (ElectronicMaterials 2003) To get thicker films, we used a double coating procedure, which can achieve the film thicknesses up to $60 \mu \mathrm{m}$ before reflow. For a film thickness above $60 \mu \mathrm{m}$, additional coating procedure can be used, however, it may result in a non-uniform film thickness with difficulty to determine accurate exposure doses. Other high viscous reflow photoresist, such as AZ P4903, is recommended for getting a thicker film.

\subsubsection{PDMS microchannels}

We examined the AZ P4620 master mold (after the reflow process) and the PDMS channels casted from the reflowed resist molds using SEM and optical microscope, respectively as shown in Figure 5.6. The enlarged images (Figure $5.6 \mathrm{~b}$-i) show the different locations of bifurcation regions through the entire channel network. As shown in those figures, the surfaces were smooth at the transitions from a larger mother channel to two smaller daughter channels in the photoresist mold (Figure 5.6b-e) as well as in the PDMS replica (Figure 5.6f-i). Additionally, local resist melting was observed along the edges of the channels possibly due to the resistsubstrate adhesion and bonding.

Two identical PDMS layers cast from the same resist mold, each of which contains a channel network with semi-circular cross-sections were treated with oxygen plasma. They were then manually aligned under the optical microscope, and bonded together to form a permanent sealing. Figure 5.7b-d showed the circular cross-section the channel network at each branching steps. The dimensions of the microchannel at different branching sections were measured, and the results showed that the multi-diameter microchannel networks were successfully fabricated. 


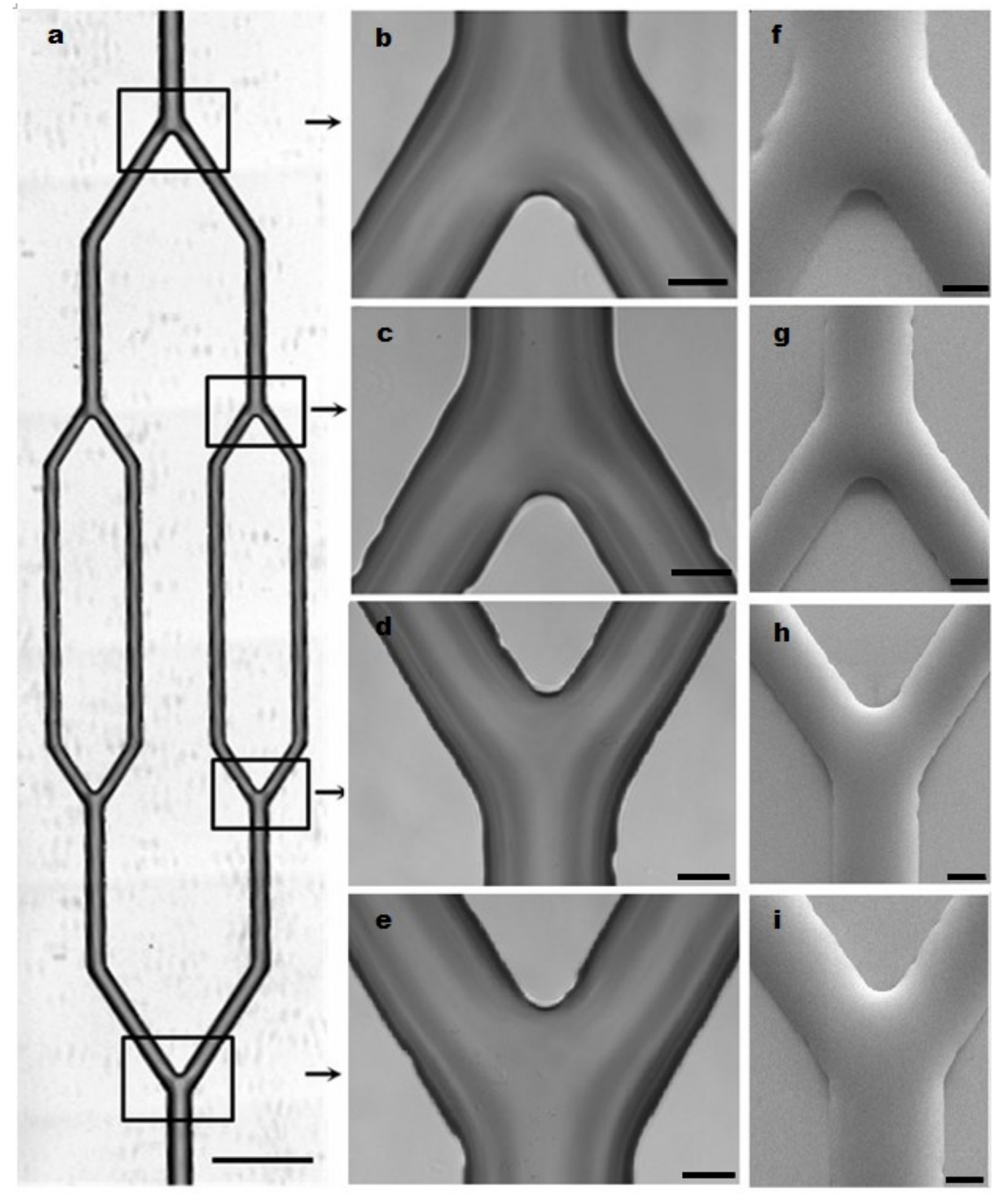

Figure 5.6 Microscope images show the smooth surface transitions at bifurcations. a) A PDMS channel network. The frames indicate the bifurcations through the channel network and the arrows indicate the panels at the right side. The scale bar is $500 \mu \mathrm{m}$. b-e) Optical images show the smooth surface transitions on the PDMS channel network. The scale bar is $50 \mu \mathrm{m}$. $\mathrm{f}-\mathrm{i}$ ) SEM images show the smooth surface transitions on the AZ P4620 resist mold. The scale bar is $50 \mu \mathrm{m}$. 


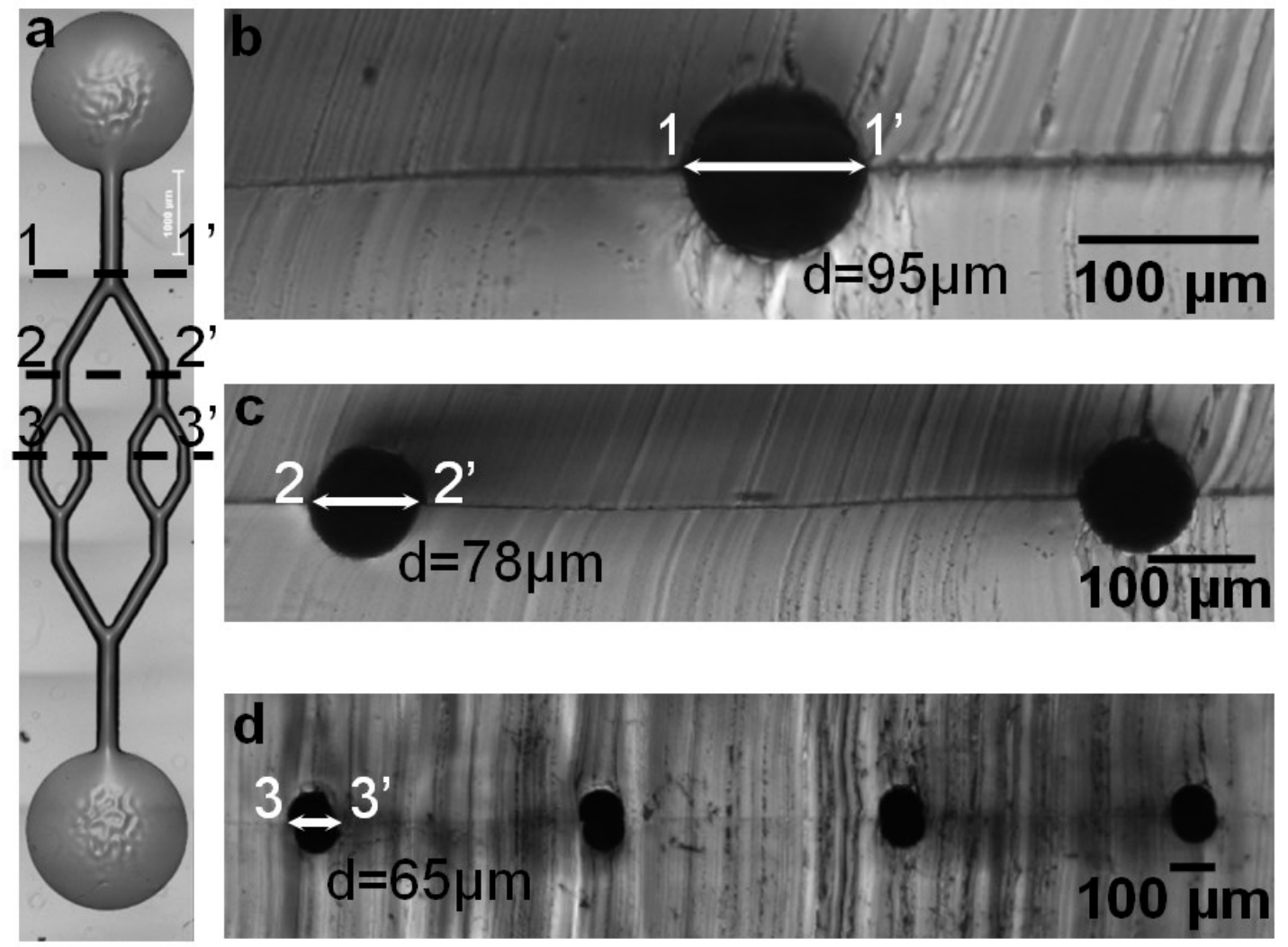

Figure 5.7 a) An aligned and bonded cylindrical microchannel network in PDMS. b-d) Circular cross-sections of PDMS molds show channel dimensions at each level (1-1', 2-2', and 3-3').

\subsubsection{Microchannel networks dimensions}

To determine the dimension variations between the designed patterns and actual fabricated channels, we compared the results of measured channel diameters before the reflow, after the reflow, and after PDMS soft lithography. To prevent the variations caused by additional fabrication factors, we kept the same PDMS mixing ratios, baking temperature, and baking duration. However, because the fabrication of different diameters of semi-circular patterns requires different photoresist film thicknesses and exposure doses, we found that channel widths were reduced (6-10\%) during the processes involved with photolithography and development as shown in table 1 . Additionally, the reflow process also decreased the channel widths by $2 \%$ on average because of photoresist volume reductions and boundary movement. (Elias 1997; O'Neill 
and Sheridan 2002) PDMS microchannel normally shrinks while curing and this shrinkage is dependent upon curing temperature, the ratio of base to curing agent, and curing time. From our results, both PDMS microchannel shrinkage and swelling occurred, and the ratio of the changes were small compared with the changes caused in the photolithographic processes. Based on the discussion above, the shrinkage of microchannels mainly occurred before the reflow. Each master mold should be designed to compensate for this shrinkage, and the fabrication parameters need to be optimized to have the fabricated channel dimensions more accurately closer to the designed dimensions. To mimic the microvascular networks in vivo based on Murray's law, a multi-level branching channels with multi-diameter at different levels are required. A directwrite method for the creation of multi-diameter microfluidic channels previously reported for the potential benefits of resembling in vivo physiological vascular conditions in a biomimetic design. (Lim, Kamotani et al. 2003) Multi-diameter channel network can lower the overall resistance and keep more uniform flow velocities throughout the network than the uniformly thickness channel generated by the planar patterning techniques. With this consideration, we examined the crosssections of the molded PDMS channels at the different branching levels in the network. The channel widths and central heights were measured using NISElements imaging software (Nikon). There might be some variations in these measurements because of the way that PDMS channels were cut. At each branching level, the central height of the channel is very close to the half width of the channel, which indicated that the shape of the reflowed photoresist could be well approximated by a semi-circular surface under well controlled conditions as shown in table 5.1. As discussed in the AZ P4620 master mold section, the reflowed channel surface profiles are determined by the design channel widths, the volume of the photoresist patterns, and reflowing process. Our results show that the photoresist reflow technique can create multi- diameter 
branching channel networks in a more convenient approach by photoresist reflow techniques, and allow for the design of microvascular biomimetic systems that obey Murray's law.

Table 5.1 The measurement of microchannel cross-sectional dimension. (unit: $\mu \mathrm{m}$ )

\begin{tabular}{|c|c|c|c|}
\hline $\begin{array}{c}\text { Designed } \\
\text { diameters }\end{array}$ & $\begin{array}{c}\text { Channel width } \\
\text { before reflow }\end{array}$ & $\begin{array}{c}\text { Diameters after } \\
\text { reflow }\end{array}$ & $\begin{array}{c}\text { Diameters in } \\
\text { PDMS }\end{array}$ \\
\hline 25 & 23.26 & 23.17 & 24 \\
\hline 50 & 46.92 & 44.67 & 46 \\
\hline 100 & 88.95 & 85.48 & 85 \\
\hline 150 & 138.96 & 137.01 & 136 \\
\hline 200 & 180.55 & 180.55 & 179 \\
\hline
\end{tabular}

\subsubsection{Long-term cell culture}

As been discussed in Chapter 3, a confluent monolayer was developed between 2 and 4 days of the HUVECs being exposed to a constant medium flow. Figure 5.8a-c shows the microscopy image of the HUVECs covering the inner surface of the microchannels at different branching regions. Figure 5.8d shows the confocal microscopy image and the circular cross-sectional view of HUVECs.

To visualize the cells after the cell culture, cells were labeled with PKH (red fluorescent dye PKH26; Sigma) and nuclei staining (NucBlue ${ }^{\mathrm{TM}}$, Invitrogen) dyes which exhibited lower cytotoxicity. (Parsa, Upadhyay et al. 2011)

The microchannel network was loaded with fixing media, (3.5\% Paraformaldehyde diluted with PBS) and then the device was submerged in fixing media and completely covered with aluminum foil and stored at $4^{\circ} \mathrm{C}$ in the refrigerator to prevent the device from drying out and bleaching. The fixed device was ready for confocal imaging. 

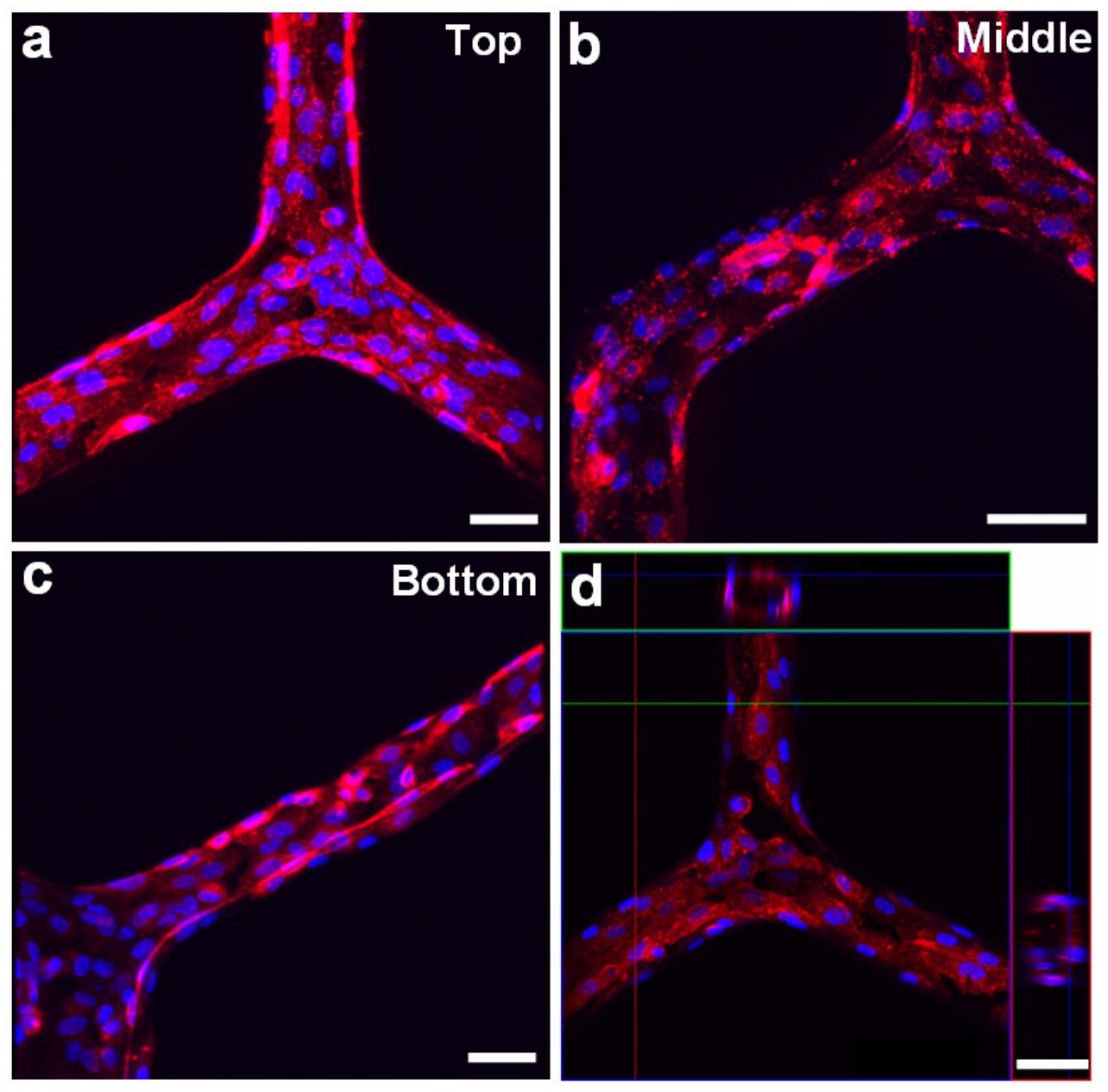

Figure 5.8 Microscopy images using fluorescent cell membrane dye (red) and cell nuclear dye (blue) show that the HUVECs line the inner surface of a cylindrical microchannel network at difference branching regions. a) Top, b) Middle, and c) Bottom. d) Confocal microscopy image shows the circular cross-sectional view of HUVECs lining around the channel network. Scale bars: $100 \mu \mathrm{m}$.

\subsection{Chapter summary}

In conclusion, an inexpensive and reproducible micromanufacturing method, combining with photolithographic reflow photoresist and PDMS soft lithography, was developed for fabricating cylindrical microchannels and multi-diameter microchannel networks with circular cross-sections. Several fabrication parameters, such as reflow parameters and shrinkage, were discussed and indicated compensation is required for microchannel design to close approximate 
microvessels. The fluorescent cell membrane staining showed that the photoresist reflow technique can create multi- diameter branching microchannel networks in a more straightforward approach, and allows us to design microvascular biomimetic systems that approximately obey Murray's Law and mimic the geometry of in vivo microvasculature, so that the overall channel resistance can be minimum. After HUVECs were seeded into the device, a long-term perfusion was performed from 4 days up to 2 weeks. The cells exhibited good proliferative and morphological conditions. The cell membrane and nuclei staining showed the entire inner surface of a cylindrical microchannel network was covered with endothelial monolayer. Thus, the developed microfluidic device provides a rapid and reproducible approach to create multidiameter microchannel networks, which mimics the geometry of in vivo microvessels. The fabricated cylindrical microchannels and networks can work either as a scaffold for investigating microvascular cells seeding and growing inside of cylindrical channels under shear flows and lumen pressures or as a mold for further fabrication of tissue scaffolds for morphogenesis and tubulogenesis. 


\section{Chapter 6 Characterization of Endothelial Junctions and Cell Morphology}

\section{Analysis in Response to Shear Stress}

In this chapter, the functionalities of developed microvessel model were validated. Adherens junctions were confirmed with VE-cadherin immunofluorescence staining. VE-cadherin is an endothelial specific adhesion molecule between the endothelial cell junctions and an important determinant of microvascular integrity in vivo. In addition, by applying different levels of shear stresses, the endothelial responses were studied with F-actin staining. The cellular morphological changes were quantified by cell alignment, aspect ratio, and surface area. The results showed that the developed microvessel model preserved key features of microvessel in vivo, such as the presence of junction protein, the alignment and elongation along the direction of the flow, and the morphology change induced by different shear stresses.

\subsection{Background}

\subsubsection{Endothelial adherens junctions}

One major function of endothelial cells is to control the infiltration of blood proteins and circulating cells into the vessel wall and the underlying tissues. (Dejana 2004) It is mediated by two pathways: transcellular and paracellular pathways. The paracellular pathway is mediated by the coordinated opening and closure of endothelial cell-cell junctions. (Figure 6.1) Among those intercellular junctions that work as the cell-cell adhesive barrier structures in the microvascular endothelium, the most frequently distinguishable two junctions are the adherens junction and tight junction. (Mehta and Malik 2006) The adherens junction presents in most microvascular beds and is the most ubiquitous type of endothelial cell to cell junction.(Stokes, Cooper et al. 2002) Tight junction is less common in microvasculature and mainly expressed in some 
specialized tissues such as blood brain barrier or blood retinal barriers. (Pardridge 2006) To validate the barrier function of the developed endothelial confluence monolayer within our microfluidic microchannel network, the adherens junction is considered an important factor.

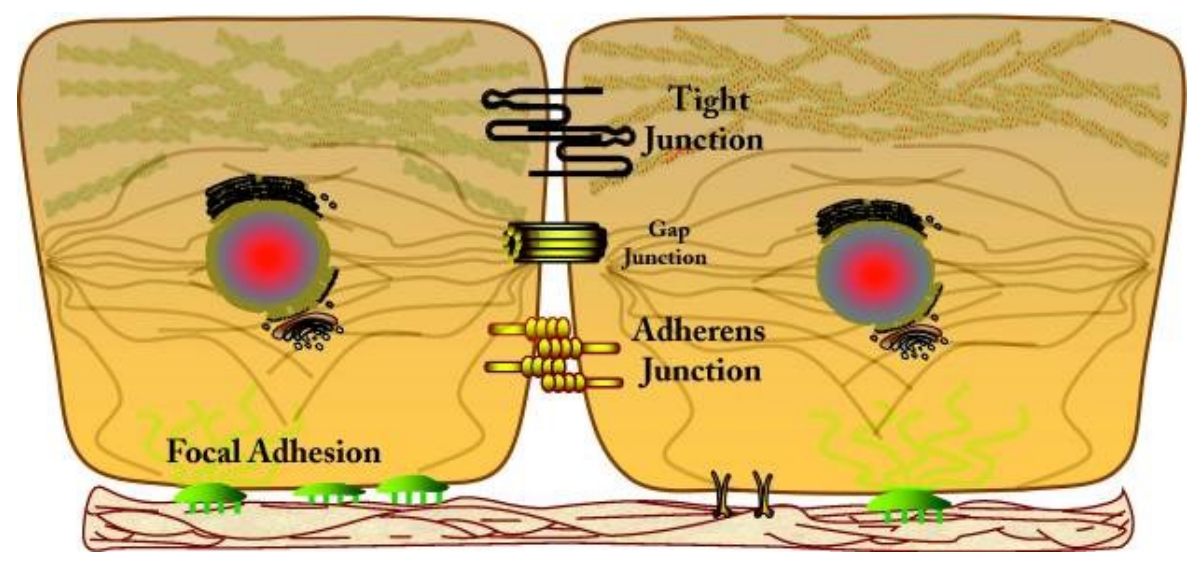

Figure 6.1 Endothelial cell junctions and adhesions. Endothelial cells of the microvessel wall are joined together by intercellular junction proteins: adherens junction, tight junction and/or gap junction. Barrier function in most vascular beds is provided by adherens junction. Only some specific microvasculatures rely on tight junction for additional barrier function, and gap junction does not directly contribute to barrier functions, but facilitate signaling between cells. (Yuan and Rigor 2010)

Vascular endothelial (VE)-cadherin performs as a strictly endothelial specific adhesion molecule between endothelial cell junctions, as well as a major determinant for the integrity of microvascular endothelium. The molecular organization of adherens junction is shown in Figure 6.2. The minimal functional unit of VE-cadherin is dimer, and EC1 to EC5 are the five homologous extracellular domains.(Gumbiner 2005) Similar to other members of the cadherin family, VE-cadherin is linked to the catenin proteins p120, $\beta$-catenin ( $\beta$ cat) and plakoglobin (plako) via the cytoplasmic tail. The $\beta$-catenin and plakoglobin are bound to $\alpha$-catenin ( $\alpha$-cat), and $\alpha$-catenin interacts with couple actin-binding proteins such as $\alpha$-actinin, ajuba, zonula occludens-1 (ZO-1). (Weis and Nelson 2006) 


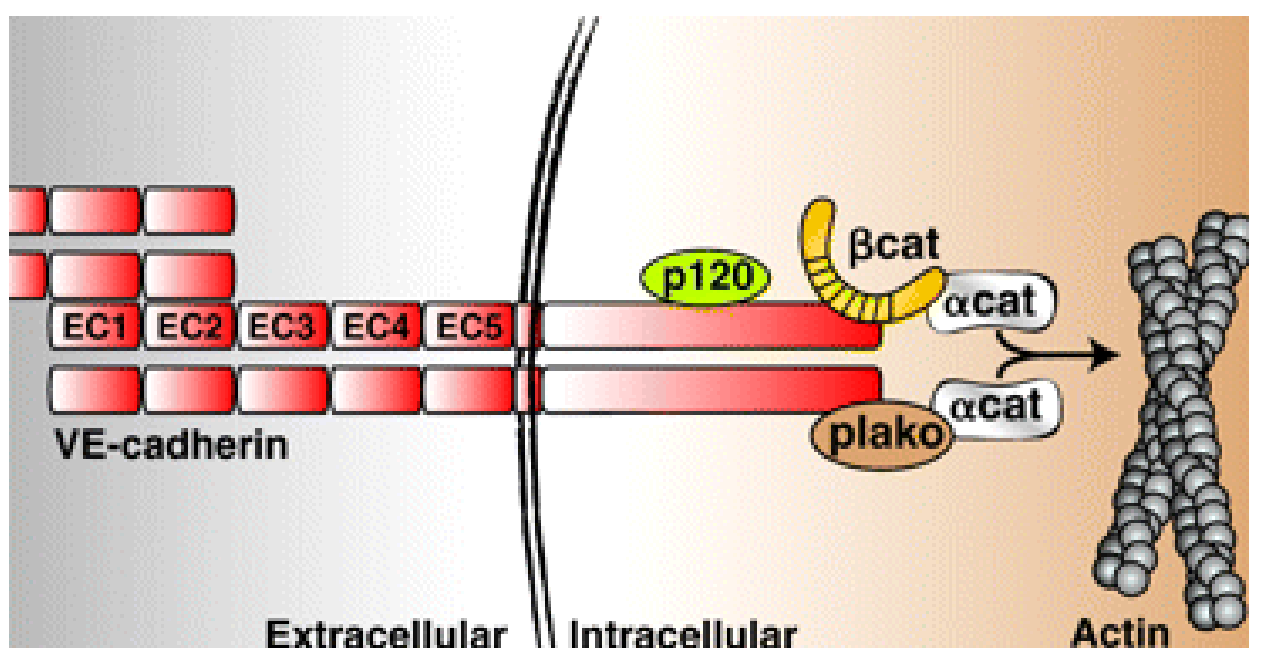

Figure 6.2 Molecular structures of endothelial adherens junctions. VE-cadherin is indicated as a dimer that is the minimal functional unit of cadherins. Five homologous extracellular domains are shown as EC1- EC5. At cell-to-cell contacts, the cluster of VE-cadherin proteins is important for certain signaling/regulating mechanism and performs as a scaffold for other complex protein clusters. The proteins that are known to interact with VE-cadherin include the catenin proteins p120, $\beta$ cat and plako. $\beta$ cat and plako is directly associated with VE-cadherin and $\alpha$-cat. (Dejana, Orsenigo et al. 2008)

It has been reported that the introduction of anti-VE-cadherin antibodies leads to a dramatic increase in permeability, vascular fragility and hemorrhages in animal models.(Corada, Mariotti et al. 1999) Permeability increasing agents were also shown to be able to target the VE-cadherincatenin complex: thrombin can change the binding of catenins to VE-cadherin;(Rabiet, Plantier et al. 1996) histamine and platelet activating factor (PAF) were able to induce tyrosine phosphorylation of VE-cadherin and $\beta$-catenin that was associated with weak junctions. (Andriopoulou, Navarro et al. 1999; Hudry-Clergeon, Stengel et al. 2005; Weis and Cheresh 2005) Antibodies can block junction reorganization up for several hours due to its strong binding with VE-cadherin. Other agents such as histamine can induce mild effects that were normally reversible. (Dejana, Orsenigo et al. 2008) The mechanisms that regulate VE-cadherin were also important for leukocyte extravasation. (Dejana 2004) In addition, it was reported that VE- 
cadherin can regulate various cellular processes such as cell proliferation, apoptosis and modulates vascular endothelial growth factor receptor functions. (Lampugnani and Dejana 1997; Lampugnani and Dejana 2007)

VE-cadherin junctions for endothelial cells cultured under static culture condition showed an intricate, lattice-like structure, which were mostly likely due to the overlapping and interdigitating regions of membrane on apposed cells. (Figure 6.3a)(Seebach, Dieterich et al. 2000) After exposed to short-term shear stress, broaden and accumulation of VE-cadherin were observed at the intercellular junctions as reported. (Figure 6.3b)(Ukropec, Hollinger et al. 2002) VE-cadherin was also shown to take some roles in the shear stress mediated signaling, such as form VE-cadherin- $\beta$-catenin-VEGFR2 complex as a shear stress transducer (Shay-Salit, Shushy et al. 2002) and the activation of nuclear factor $\kappa B$ and proinflammatory target genes. (Tzima, Irani-Tehrani et al. 2005) After longer or continuous laminar perfusion, thin and uniform bands of adherens junction proteins were shown at the endothelial cell interfaces. (Figure 6.3c)(Ukropec, Hollinger et al. 2002) The VE-cadherin structure after exposure to long-term shear stress was more close to in intact perfused venules as shown in Figure 6.3d. (Yuan and He 2012)

Many in vitro studies on endothelial cells responses to shear stresses were performed by seeding a high density of cells and applying shear stress within short period of time ( $<24$ hours). (Shyy, Hsieh et al. 1994; Malek and Izumo 1996; Tzima, Irani-Tehrani et al. 2005) Many of those studies were either lacking the characterization of adherens junction, or not presenting the thin and uniform bands of adherens junction proteins due to the limitation of short-term culture. Additionally, because the cells reached proliferation stagnation shortly after the high density seeding, there was increased chance of over-confluence of the endothelial monolayer. (Xia, 
Prawirasatya et al. 2011) For our microfluidic microvessel model presented here, it is able to apply long-term perfusion to maintain the appropriate cell spreading, viability, proliferation and barrier function that is more close to the in vivo conditions.
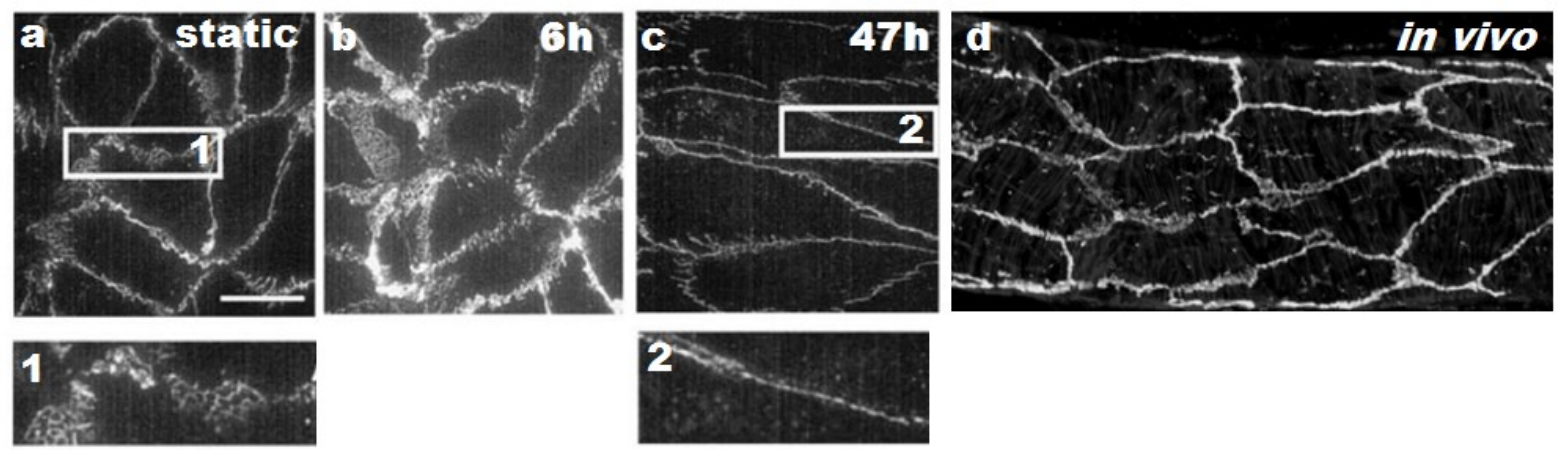

Figure 6.3 a) The VE-cadherin staining of HUVEC maintained in static culture condition. b) After exposed to shear stress at $10 \mathrm{dyne} / \mathrm{cm}^{2}$, the intensity of VE-cadherin staining was increased, and the intricate latticework appeared to broaden. c) By 47 hours, the VE-cadherin was reorganized into compact junctions at the endothelial cell interfaces. 1 and 2 are the $2 \times$ magnifications of the boxed areas indicated in a and c. Scale bar: $25 \mu \mathrm{m}$. (Ukropec, Hollinger et al. 2002) d) VE-cadherin staining of intact venule from animal model. (Yuan and He 2012)

\subsubsection{Cell morphology analysis in response to shear stress}

The actin cytoskeleton is essential for many cellular processes such as migration, morphogenesis, cytokinesis, endocytosis and phagocytosis. (Richards and Cavalier-Smith 2005) The physiological role for actin filaments of cells is to provide internal mechanical supports, tracks of movement of intracellular materials, and forces to drive cell movement from hydrolyzing ATP by myosin motor proteins. (Ge, Deng et al. 2009; Pollard and Cooper 2009)

Figure 6.3 shows the structures of actin and diagrams of fundamental reactions. (Pollard and Cooper 2009) Under the regulation by actin-binding proteins, actin monomers spontaneously polymerize into long and stable filaments. (Figure $6.3 \mathrm{a}-\mathrm{b}$ ) (Bugyi and Carlier 2010) Actin filaments are confined to certain length, and finally assemble and cross-link into networks or 
bundles by nucleation, elongation, branching and crosslinking. (Figure $6.3 \mathrm{c}-\mathrm{f}$ ) After the formation of protrusive networks, the actin filaments form the contractile structures with myosin motors such as cytokinetic contractile ring, myofibrils of muscle cells and stress fibers of nonmuscle cells. (Figure $6.3 \mathrm{~g}$ )(Michelot and Drubin 2011)

a

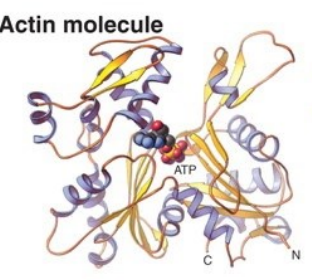

b Spontaneous nucleation and elongation

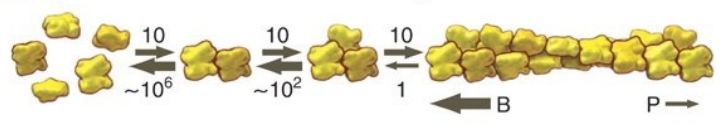

C Monomer binding

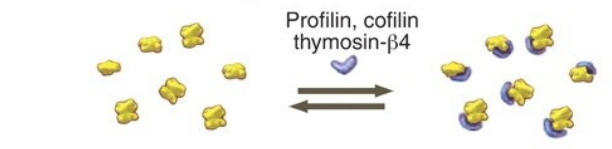

d Nucleation and elongation by formin

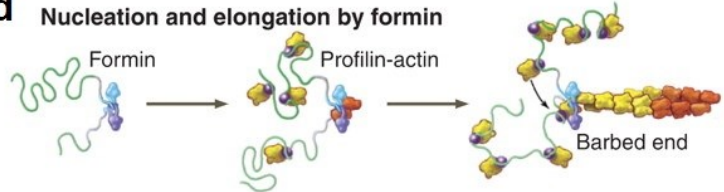

e Branching nucleation by Arp2/3 complex

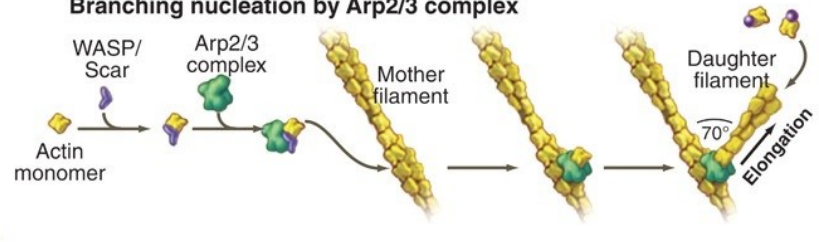

f
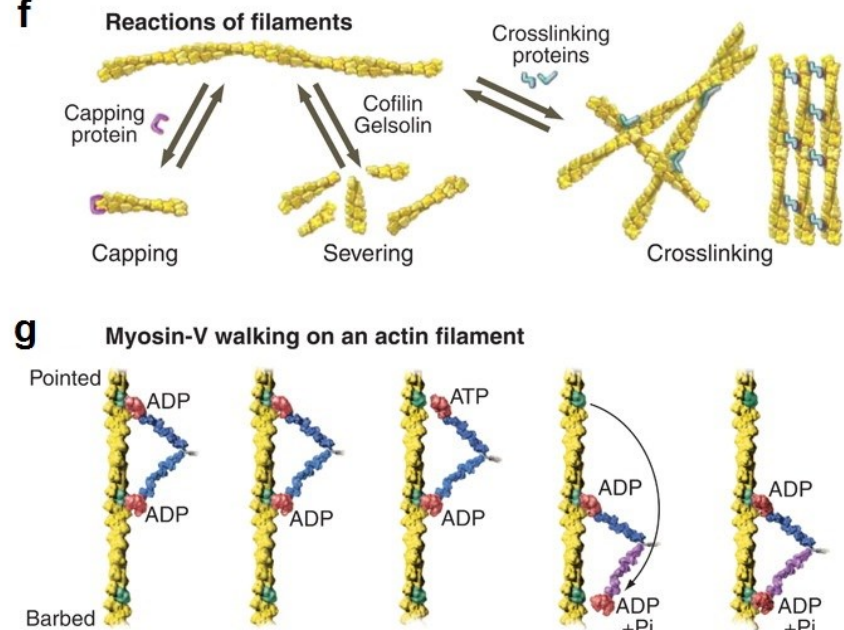

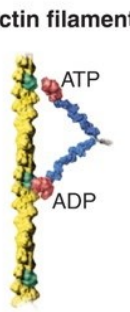

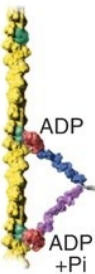

Figure 6.3 Structures of actin and diagrams of fundamental reactions. a) Ribbon and space-filling models of the actin molecule (pdb:1ATN). b) Spontaneous nucleation and elongation. Longer polymers grow rapidly at the barbed end (B) and slowly at the pointed end (P). c) Actin monomer binding various proteins. d) Nucleation and elongation by formins. e) Nucleation by Arp2/3 complex. f) Reactions of actin filaments. Capping proteins bind to and block barbed ends; cofilin and gelsolin sever filaments; cross-linking proteins assemble networks and bundles of actin filaments. g) Myosin motors, such as myosin V, use cycles of ATP hydrolysis to walk along actin filaments. (Pollard and Cooper 2009)

Contractile stress fibers are the prominent actin filament structures in endothelial cells. (Tojkander, Gateva et al. 2012) Under fluid shear stress, endothelial cells not only show elongation and orientation in the flow direction, but also appear to have thick stress fibers that 
align along the long axis of the cells. (Sato and Ohashi 2005; Shao, Wu et al. 2009; Millán, Cain et al. 2010) The abnormal of actin dynamics is associated with certain pathological disorders such as cancer and myofibrillar myopathies. (Rousseau, Houle et al. 1997; Wójciak-Stothard, Williams et al. 1999; Tzima, Reader et al. 2003; Davies 2008)

In previous studies, cultured endothelial cells demonstrated significant reduced in contact areas under a higher shear stress, in which cells tended to reduce the width of the upstream face to decrease the area of cells exposed to oncoming fluid in order to reduce the effect of shear stress. (Galbraith, Skalak et al. 1998) It also has been reported that endothelial cells required over 48 hours to reach steady state when they experience shear stress changes. (Noria, Cowan et al. 1999) Within the first 24 hours of shear stress changes from low to high, there were delays in cytoskeleton reconstruction and reorientation in response to higher flow shear stresses in confluent endothelial monolayer because cells in confluent situation were constrained by each other due to the connections within neighboring cells. (Galbraith, Skalak et al. 1998)

The activation of integrin $\alpha v \beta 3$ was reported in bovine aortic endothelial cells induced by shear stress, followed by the increased binding between $\alpha v \beta 3$ and extracellular cell matrix proteins. This binding further induced a transient inhibition of Rho that was necessary for cytoskeletal alignment. (Tzima, del Pozo et al. 2001; Shiu, Li et al. 2004; Zeng and Tarbell 2014) Other sensing mechanisms have also been studied such as platelet endothelial cell adhesion molecule-1, adherens junctions, ion channels such as shear stress responsive $\mathrm{K}+$ channel and a sodium channel that was similar to SCN4 and SCN 8, caveolae and G proteins. (Resnick, Yahav et al. 2003) The cytoskeleton provided a structural framework for the mechanical forces transmission between the luminal, abluminal and junctional surfaces. It also transduced force 
signaling to several interior cell compartments including the cytoplasm, the nucleus, and focal adhesion sites. (Li, Haga et al. 2005)

To examine the morphological change of endothelial cells, the microvessel models were exposed to different shear stresses under long-term perfusion. With numerical simulation, the shear stress distribution was able to recapitulate the in vivo conditions in venules. The endothelial responses were studied with F-actin staining, and quantified by the percentage of cell alignment, aspect ratio, and cell surface area.

\subsection{Method}

\subsubsection{Immunofluorescent staining}

HUVECs were seeded into the microfluidic device and reached confluence after $4 \sim 7$ days of perfusion. The devices were fixed in $2 \%$ paraformaldehyde solution (Electron Microscopy Science) for 30 minutes by perfusing the fixing solution into the network. The cells were blocked with $1 \mathrm{mg} / \mathrm{ml}$ bovine serum albumin (BSA, Sigma) in PBS solution for 30 minutes followed by permeabilization with $0.1 \%$ Triton X-100 (Sigma) for 5 minutes.

The primary antibody (VE-cadherin) was perfused at $4{ }^{\circ} \mathrm{C}$ for overnight. Then, the second antibody (Alexa488, Invitrogen) was perfused for 1 hour at the room temperature. Finally, the cell nuclei were stained with DRAQ 5 (Biostatus). Fluorescent images were obtained using an inverted optical microscope (Nikon Ti-E inverted live-cell imaging microscope) and a confocal laser-scanning microscope (Leica TCS SL), respectively.

When the concentration of paraformaldehyde was over $4 \%$, or the fix solution was perfused into the microvessel network too rapidly, such as by vacuum suction (up to $1.8 \mathrm{ml} / \mathrm{min}$ ), there were breakdowns and tearing of VE-cadherin junctions as shown in Figure 6.4a. This issue was 
solved by keeping the concentration of paraformaldehyde at $2 \%$ and loading the fix solution with a syringe pump system at a slow perfusion rate. $(0.35 \mu \mathrm{L} / \mathrm{min}$, Figure $6.4 \mathrm{~b})$
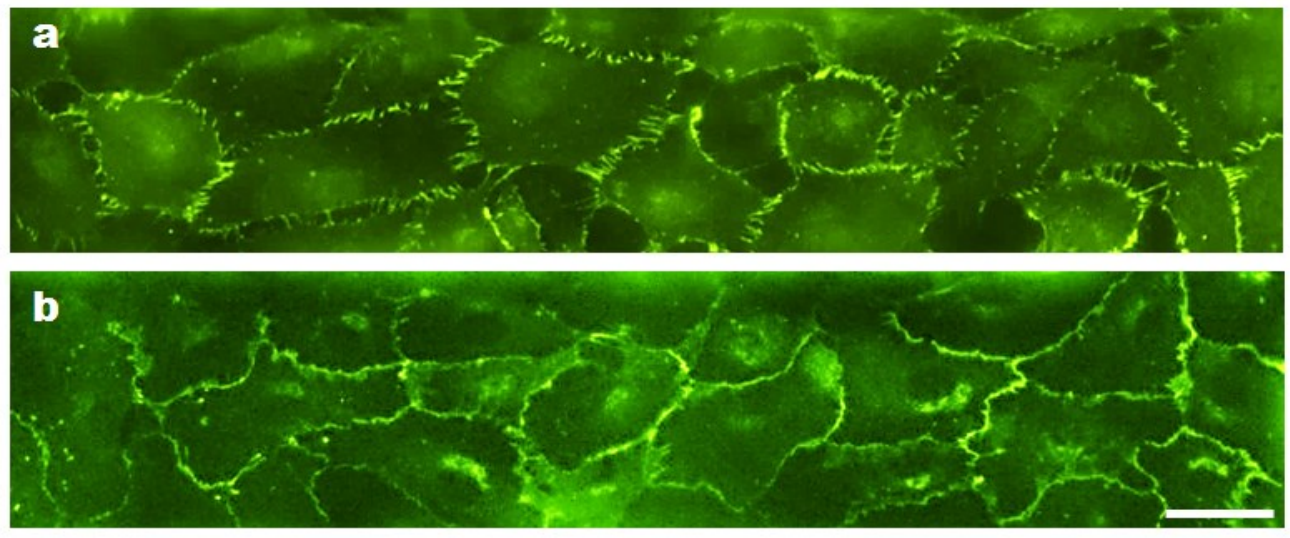

Figure 6.4 a) The breakdown and tearing of VE-cadherin after fixing, as VE-cadherin shows dashed line connecting cells. b) After probably adjust the concentration of fixing media and loading speed, the VE-cadherin was distributed normally. Scale bar: $50 \mu \mathrm{m}$.

\subsubsection{Analysis of cell morphology in response to shear stress}

To study the actin cytoskeleton and HUVECs morphological changes under shear stresses, different scenarios were performed to vary the culture and shear flow conditions, which have been used to study the interactions of endothelial cells-shear stresses. The detail experimental conditions are listed in Table 6.1. Briefly, after initial seeding three different flow conditions were set for the same patterned microvessel network in different devices: low shear culture, low shear test (LSC-LST); low shear culture (till the endothelial cells reached confluence), high shear test (LSC-HST); and high shear culture, high shear test (HSC-HST). The transition from low shear stress to high shear stress was gradually applied by programming a step function (10 steps of increase in 18 hours) using the syringe pump. (Harvard Apparatus)

The fixing, blocking and permeabilizing procedure of F-actin staining was the same as VEcadherin staining. The microvessel models were then stained with Phalloidin-Alexa 633 (Sigma) 
for 10 minutes, followed by the DRAQ5 nuclei staining. Fluorescent images were obtained using a confocal laser-scanning microscope (Leica TCS SL). In order to clearly label the stress fiber as shown in Figure 6.5b, the permeabilization was performed for 5 minutes with $0.1 \%$ Triton X-100. Long treatment of Triton can increase the nonspecific background staining. (Capani, Martone et al. 2001) 10 minutes of permeabilization caused much less details that were shown of the stress fibers. (Figure 6.5a)

Table 6.1 Summary of flow rate test.

\begin{tabular}{|c|c|c|c|c|}
\hline & $\begin{array}{c}\text { Culture and test } \\
\text { condition }\end{array}$ & $\begin{array}{c}\text { Flow rate for } \\
\text { culture/test }\end{array}$ & $\begin{array}{c}\text { Wall shear stress at the } \\
\text { selected region }\end{array}$ & $\begin{array}{c}\text { Total number } \\
\text { of cells }\end{array}$ \\
\hline LSC-LST & $\begin{array}{c}\text { Low shear culture- } \\
\text { Low shear test }\end{array}$ & $\begin{array}{c}0.35 \mu \mathrm{L} / \mathrm{min} / \\
0.35 \mu \mathrm{L} / \mathrm{min}\end{array}$ & $1.0 \mathrm{dyne} / \mathrm{cm}^{2}$ & 207 \\
\hline \multirow{2}{*}{ LSC-HST } & $\begin{array}{c}\text { Low shear culture- } \\
\text { High shear test }\end{array}$ & $\begin{array}{c}0.35 \mu \mathrm{L} / \\
4.05 \mu \mathrm{L} / \mathrm{min}\end{array}$ & $\begin{array}{c}1.0 \mathrm{dyne} / \mathrm{cm}^{2}, \\
\text { then } 10 \mathrm{dyne} / \mathrm{cm}^{2}\end{array}$ & 209 \\
\hline HSC-HST & $\begin{array}{c}\text { High shear culture- } \\
\text { High shear test }\end{array}$ & $\begin{array}{c}4.05 \mu \mathrm{L} / \mathrm{min} / \\
4.05 \mu \mathrm{L} / \mathrm{min}\end{array}$ & $10 \mathrm{dyne} / \mathrm{cm}^{2}$ & 235 \\
\hline
\end{tabular}
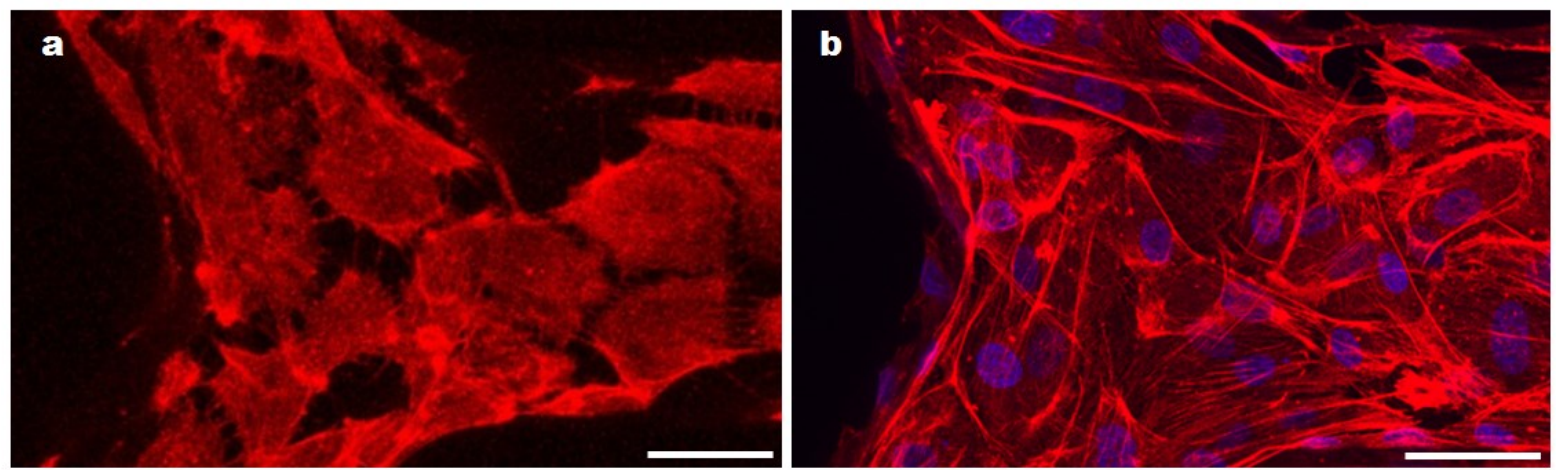

Figure 6.5 Comparing different permeabilizing protocols of the F-actin staining. a) When the cells were treated with Triton for 10 minutes, there was a significant level of nonspecific background staining. b) After 5 minutes of Triton treatment, image quality of the microscopy was greatly improved. The images show the same branching region from two different devices with different permeabilizing duration. Scale bar: $50 \mu \mathrm{m}$.

Quantitative analysis was performed to examine HUVEC morphology changes (i.e. aspect ratio and cell surface area) in responses to different levels of shear stresses. The aspect ratio was 
defined as the length of the primary axis $\left(\mathrm{d}_{1}\right.$, along the flow direction) divided by the length of the secondary axis ( $\mathrm{d}_{2}$, perpendicular to the flow direction). A cell was considered as an ellipse shape and the corresponding cell surface area was calculated as $\pi d_{1} d_{2} / 4$. For statistical analysis, data was presented as the mean \pm standard error (SE) and each individual experiment was performed at least three times $(n \geq 3)$. The results were evaluated by the $t$ test and single factor analysis of variance (ANOVA).

\subsubsection{Numerical simulations}

Computational fluid dynamics (CFD) has been widely used to simulate and optimize the microfluidic device design. (Glatzel, Litterst et al. 2008; Adeosun and Lawal 2009; Wei and Bai 2013) The advantages of such numerical method include the possibility to determine values of parameters that cannot be experimentally measured, proof the feasibility of design, validate analytical calculations, and elucidate experimental results. (Boy, Gibou et al. 2008)

\subsubsection{Governing equation and boundary conditions}

From a fluid-mechanical standpoint, the fundamental properties of micro/nanoscale fluid flow are different from those fluid conditions in macroscale devices. Interfacial phenomena such as surface tension and wetting (Darhuber and Troian 2005; Hu and Li 2007) and electrokinetic phenomena such as electrophoresis, sedimentation potential, electrosmosis, and streaming potential (Bazant and Squires 2004) become more dominant than gravity and pressure. Based on different analytical objects, the numerical models often use modified continuum governing equations and non-slip boundary conditions to account for those forces. (Kirby 2010) For example the fluidic phenomena in the microchannel (the dimension is larger than $100 \mathrm{~nm}$ ) still can be described by continuum Navier-Stokes equations. There are several commercial softwares available to build such CFD model, e.g., COMSOL, Fluent, Ansys, CFX, and Coventor. 
To simplify the Navier-Stokes equations, the flow field within the microfluidic channel was considered as a single phase, non-compressible, Newtonian fluid with low Reynolds number (Re). Newtonian fluid means the viscous stresses induced by the flow field are always linearly proportional to the local strain rate, which is the rate of change of the fluid body deformation over time. In fluid dynamics, the Reynolds number (Re) is often used to determine the flow pattern of viscous flow. This dimensionless parameter is defined as: $R e=\frac{\rho V L}{\mu}(6.1)$, where $\rho$ is the density of the fluid, $\mathrm{V}$ is the mean velocity, $\mathrm{L}$ is the characteristic linear dimension, and $\mu$ is the dynamic viscosity of the fluid. In the case of flow chamber, the low Reynolds number $(\operatorname{Re}<$ 1000) indicates laminar flow with steady streamlines.

Continuity equation represented the conservation of mass: $\rho \nabla v=0$ (6.2). And the vector equation represented the conservation of momentum: $\rho \frac{\delta v}{\delta t}+\rho(v \cdot \nabla) v=\nabla \cdot[-\rho I+$ $\left.\mu\left(\nabla \mathrm{v}+(\nabla \mathrm{v})^{T}\right)\right]+F(6.3)$, where $\rho$ is the density, $v$ is the velocity vector, $p$ is the pressure, and $F$ is the volume force vector, which can be used to incorporate the effects of volume force such as gravity.

The common boundary condition for a stationary solid wall was no slip: $v=0(6.4)$. There were other boundary conditions such as slip wall, sliding wall, moving wall, and leaking wall. (Poinsot, amp et al. 1992; Stone, Stroock et al. 2004)

\subsubsection{3D Numerical model for microchannel networks devices}

For the morphology change of endothelial cells, shear flows and associated lumen pressures are critical parameters that affect vessel maturation and structure, barrier functions and permeability, and regulation of key enzymes. (Meeson, Palmer et al. 1996; Schaper 2001; van Royen, Piek et al. 2001; Tarbell 2010; Potter, Lundberg et al. 2011) To analyze the 
spatiotemporal variations of the fluid flows and pressure distributions inside the microchannels and networks, a fluid dynamic analysis model using COMSOL multiphysics software (Version 4.0.0.982, COMSOL Inc. USA) was built to numerically simulate shear flows and pressure distributions. The fluid body was considered as a stationary case, and culture medium was selected as the reference fluid during the simulation, a constant density $\left(1060 \mathrm{~kg} / \mathrm{m}^{3}\right)$ and a constant dynamic viscosity $\left(0.001 \mathrm{~N} \cdot \mathrm{s} / \mathrm{m}^{2}\right)$ were set. The procedures to implement the CFD analysis were listed as follows:

1. Selecting space dimension. 2. Adding and setting the model as laminar flow and stationary. 3. Defining length unit as micrometer $(\mu \mathrm{m})$. 4. Importing the pattern file from AutoCAD (Autodesk, Inc. USA). 5. Defining the fluid properties. 6. Defining the appropriate boundary conditions (the combinations between inflow conditions (pressure, velocity, or flow rate) and outflow conditions (pressure, velocity, or flow rate), and no-slip wall). 7. Creating mesh. 8. Under the study menu, selecting PARallel sparse DIrect linear SOlver (PARDISO) and executing the calculation.

PARDISO, an embedded solver in COMSOL, was used to solve the partial differential equation (PDE) system governed by the Navier-Stokes equations for a Newtonian and noncompressible fluid. (Stalder, Liu et al. 2011) The meshing was realized by using the free triangular function that automatically created an unstructured triangular mesh of the subdomain. Free triangular function has the flexibility to control the number, size, and distribution of elements based on the available computing capability of the working computer. The total number of subdomain elements used in the simulation was around 100,000 with the maximum element size of $10 \mu \mathrm{m}$. In order to analyze shear stress details at the certain boundary regions of interest, 
such as curvatures along the network, resolution of curvature was adjusted to generate a finer mesh along curved boundaries.

With the CFD simulation model, the flow rates and corresponding shear stress were simulated as shown in Figure 6.6. The shear stresses were obtained by COMSOL simulation based on $\tau=\gamma \cdot \mu(6.5)$, where $\tau(\mathrm{Pa})$ is the wall shear stress, $\gamma(1 / \mathrm{s})$ is local shear rate, and $\mu$ is the dynamic viscosity $\left(\mathrm{N} \cdot \mathrm{s} / \mathrm{m}^{2}\right)$. (Couzon, Duperray et al. 2009) The simulation result provided a good guidance for the following cell culture and testing experiment.

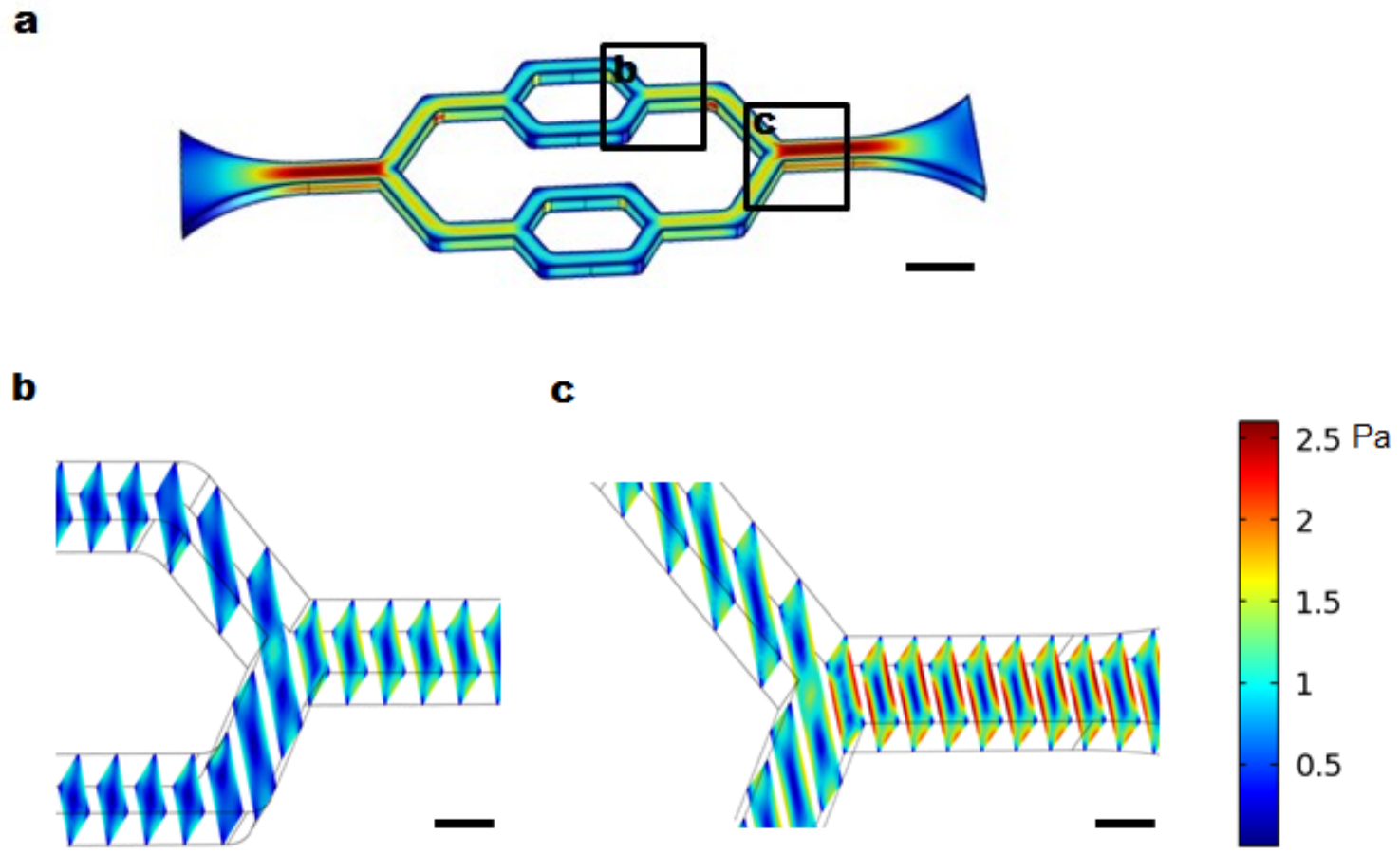

Figure 6.6 The numerical 3D model built with CFD commercial software COMSOL. a) Wall shear stress distribution of the whole networks, where the selected regions of $\mathrm{b}$ and $\mathrm{c}$ were indicated. Scale bars: $500 \mu \mathrm{m}$. b-c) The cross-section view of the shear stress at different branching section. Scale bars: $100 \mu \mathrm{m}$. 


\subsection{Result and discussion}

\subsubsection{Endothelial adherens junctions}

VE-cadherin was immune-stained in the entire microchannel. Figure 6.7 shows the confocal images of VE-cadherin and nuclei staining at different regions of the microchannel. The confocal images illustrated that VE-cadherin has been well developed throughout the entire network (Figure $6.7 \mathrm{~b}, \mathrm{c}, \mathrm{d}$ ), demonstrating a continuous distribution between ECs with less spikes that often appeared in statically cultured endothelial monolayers. (Comparing with Figure 6.3a)(Orsenigo, Giampietro et al. 2012)

a
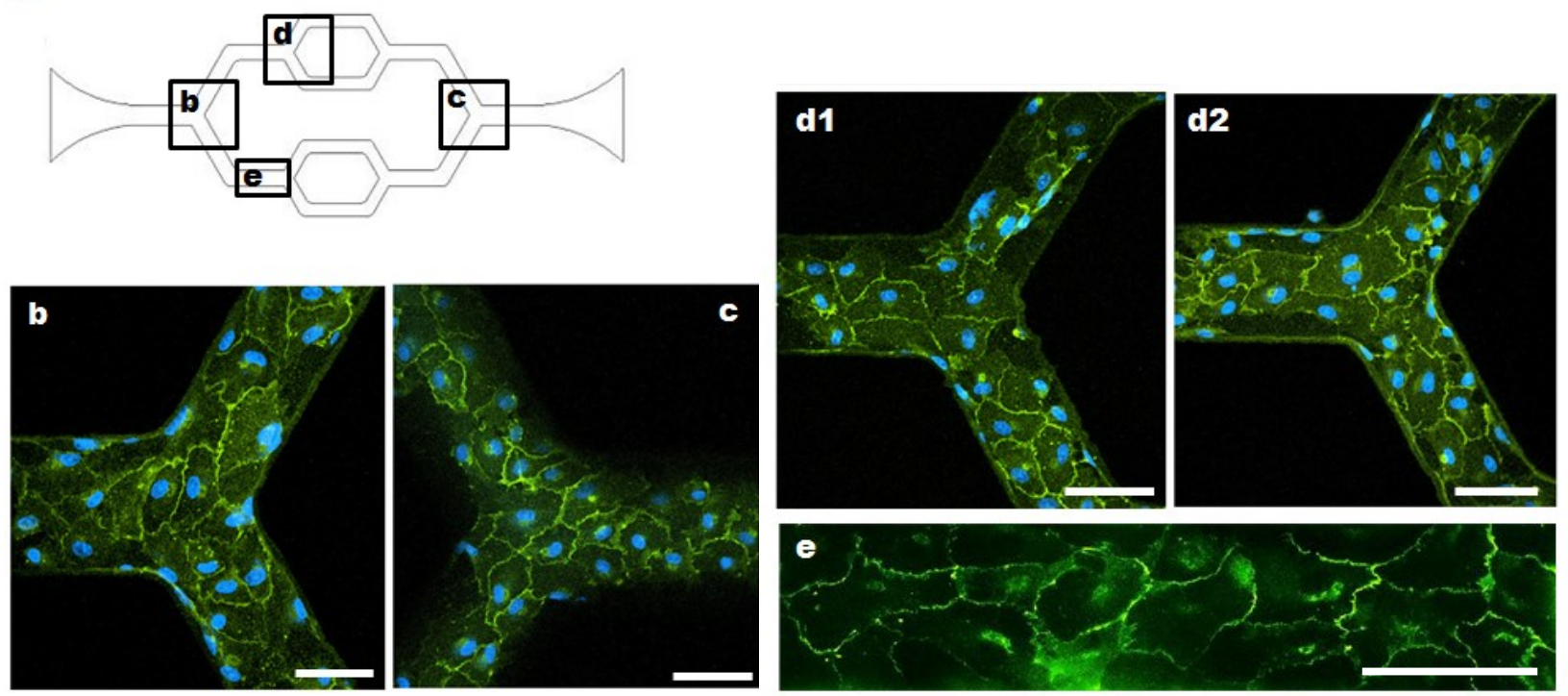

Figure 6.7 The representative immunofluorescent confocal images demonstrated the VECadherin junctions formation throughout the entire network. a) The schematic image of the network with selected regions as shown in b-d. b-c) VE-Cadherin and cell nuclei staining were shown at the first and third branching regions. $d_{1}-d_{2}$ ) VE-Cadherin and cell nuclei staining were shown at the top and bottom surfaces of the second branching regions, respectively. e) Enlarged region indicated in a. Scale bars: $100 \mu \mathrm{m}$ 
The endothelial cells that grown under flow conditions formed better monolayers with welldeveloped junctions between endothelial cells. As shown in Figure $6.7 d_{1}-d_{2}$, the HUVECs completely covered the top and bottom surfaces of the channels including the corners and formed a completely enclosed network. Most importantly, the VE-cadherin staining showed a uniformly distributed band between endothelial cells within the network, which is similar to that observed in intact venules as shown in Figure 6.3d (Yuan and He 2012; Yuan, Xu et al. 2014) and distinct from the intricate lattice-like structure commonly found in statically cultured endothelial monolayers. (Ukropec, Hollinger et al. 2002)

\subsubsection{Cell morphology analysis in response to shear stress}

To quantify the shear stresses, which the endothelium lining the microchannels experienced under our experimental conditions, we conducted the numerical simulation. The calculated wall shear stresses were $1.0 \mathrm{dyne} / \mathrm{cm}^{2}$ under the flow rate $0.35 \mu \mathrm{L} / \mathrm{min}$, and $10 \mathrm{dyne} / \mathrm{cm}^{2}$ under the flow rate $4.05 \mu \mathrm{L} / \mathrm{min}$ within the selected regions in the devices. The $3 \mathrm{D}$ simulation of the wall shear stress distribution within the network is shown in Figure 6.6.

Flow related shear stress has been shown to induce changes in cell shape and cytoskeletal structure of vascular ECs in vivo and in vitro. (Langille, Graham et al. 1991; Sato and Ohashi 2005) Under the LSC-LST conditions, about $70 \%$ of the cells show cobblestone pattern with dominated peripheral F-actin, and only $30 \%$ of the HUVECs showed elongated cell shape with increased central stress fibers aligned along the flow direction. Under LSC-HST and HSC-HST conditions, about $50 \%$ of the cells were elongated with distinct stress fibers along the flow direction. The increased number of endothelial cells that aligned along the higher flow rates indicated that the cell responded to the increased shear stresses. In each scenario, the cell shape aspect ratio in non-aligned cells was about $1.5 \pm 0.03$ and the aligned cells was $3.97 \pm 0.19,3.93$ 
\pm 0.22 and $3.95 \pm 0.19$ in LSC-LST, LSC-HST, and HSC-HST, respectively. Figure 6.8c-e shows representative images from each group and the quantifications of the cell dimensions. As for the cell surface areas, those aligned cells in high shear stress test groups (LSC-HST and HSC-HST) demonstrated significantly reduced surface areas $\left(1855 \mu \mathrm{m}^{2}\right.$ and $\left.1234 \mu \mathrm{m}^{2}\right)$ compared to those cells in low shear stress test case (LSC-LST, $2202 \mu \mathrm{m}^{2}$, Figure $6.8 \mathrm{f}$ ).
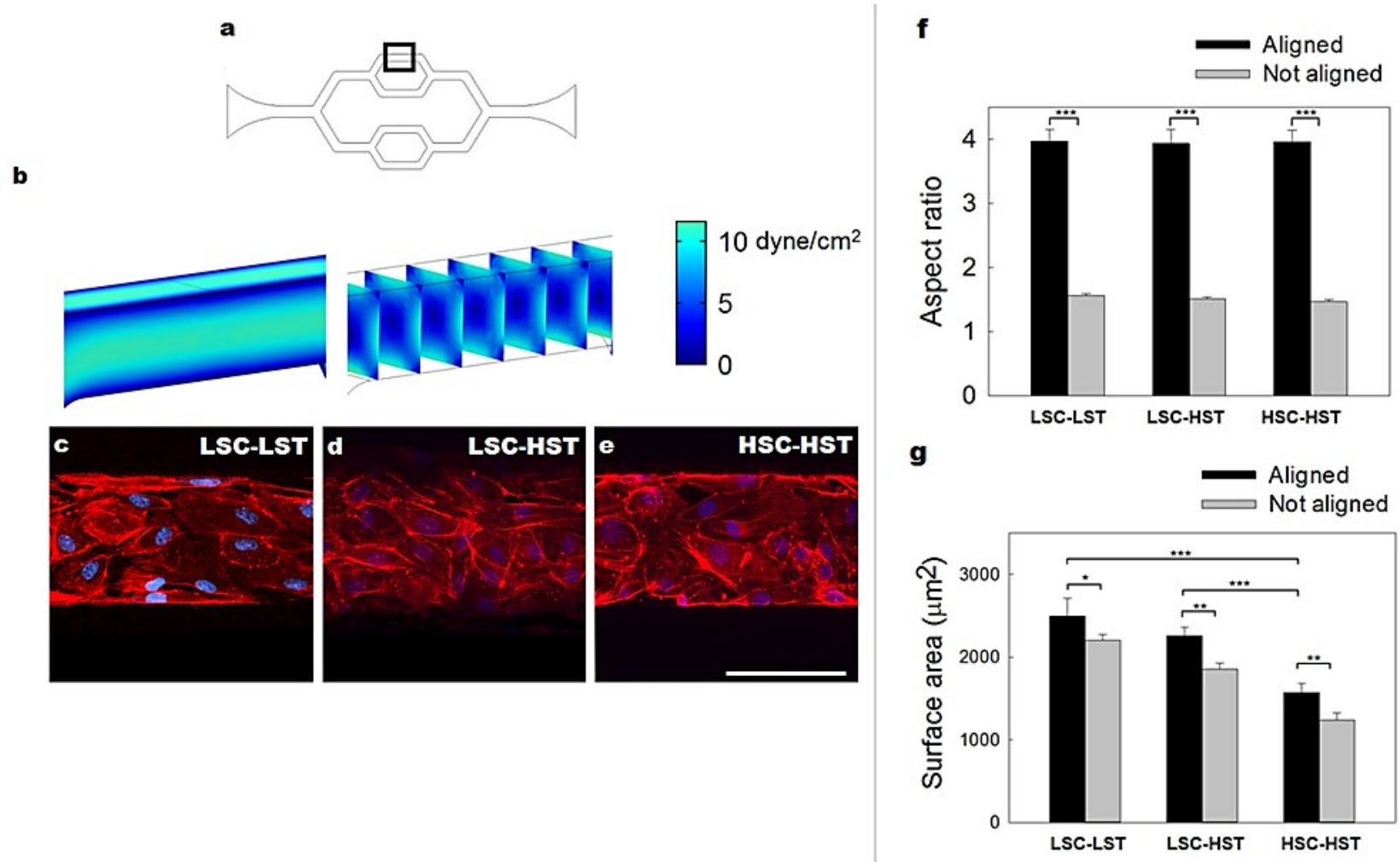

Figure 6.8 HUVECs response to the different levels of shear stresses: The morphology change was studied with F-actin staining. a) The schematic image of the network indicated with selected region for shear stress test. b) COMSOL simulation showed the wall shear stress distribution in the selected region along the network. c-e) Confocal image showed the F-actin stress fibers under the LSC-LST, LSC-HST, and HSC-HST respectively. f) The aspect ratio was compared between the three testing groups. g) Comparing of the cell surface areas between the testing groups. All data are reported as the means \pm SE of independent experiment. Scale bars: $100 \mu \mathrm{m}$. *: $\mathrm{P}<0.05 ; * *: \mathrm{P}<0.01 ; * * *: \mathrm{P}<0.001$. 
Previous studies of the endothelial F-actin rearrangement under shear stress are commonly performed in flow chambers, (Ukropec, Hollinger et al. 2002; Tzima, Irani-Tehrani et al. 2005) and cone-and-plate viscometer. (Nagel, Resnick et al. 1994; Morawietz, Talanow et al. 2000; Feugier, Black et al. 2005) The shear stresses applied in those models were either too low to be physiologically relevant, not uniformly distributed, or less versatile to be adjusted to different ranges to closely mimic the in vivo conditions. The endothelial cells usually reached confluence under static culture and then were exposed to higher shear stresses. Under higher flow conditions, majority of ECs oriented along the flow direction and demonstrated polygonal shapes, which are closer to the cell morphology reported in venular vasculature. (McDonald 1994; Orsenigo, Giampietro et al. 2012; Xu, Zhou et al. 2013) The gentle flow during the cell seeding and the continuous perfusion during the culture might be able to remove the endothelial cells that were not fully attached and prevent their over-confluence. Further studies, including the transient change of cell shape, alignment and microfilament network remodeling, could be performed to investigate endothelial cells' responses to shear stresses changes within shorter period.

\subsection{Chapter summary}

To validate the functionalities of developed microvessel model, the formation of adherens junctions was confirmed with VE-cadherin immunofluorescence staining. VE-cadherin is an endothelial specific adhesion molecule between the endothelial cell junctions and an important determinant of microvascular integrity in vivo. So far there were limited report showing the uniform and continuous VE-cadherin proteins between the endothelial cell junctions, as shown in Figure 6.7, which was more close the in vivo microvessels. Endothelial cells are also sensitive to their biomechanical environment, especially to the shear stress imposed on them by the flow. It has been reported the regulation of endothelial membrane and cytoskeletal properties, receptor 
expression, cell signaling and gene expression by shear stresses. In this work, three different levels of shear stresses were applied to the microvessel model and the morphology change of endothelial cells was studied with F-actin staining. The increased percentage of cell alignments along the direction of flow, and the decreased cell surface area under higher shear stress have been reported by the previous studies with similar results. (Galbraith, Skalak et al. 1998; Noria, Cowan et al. 1999) 


\section{Chapter 7 ATP stimulated $\left[\mathrm{Ca}^{2+}\right]_{\mathrm{i}}$ and NO production}

The regulation of the endothelial barrier function by $\left[\mathrm{Ca}^{2+}\right]_{\mathrm{i}}$ and $\mathrm{NO}$ was studied and present in this chapter. The possible signal transduction pathways of endothelial $\left[\mathrm{Ca}^{2+}\right]$, and $\mathrm{Ca}^{2+} / \mathrm{CaM}$ dependent NO synthase was briefly discussed. ATP was chosen as the representative agonist to study receptor-mediated changes in endothelial $\left[\mathrm{Ca}^{2+}\right]_{\mathrm{i}}$ and $\mathrm{NO}$ production. Two fluorescent probes (DAF-2 and Fluo-4) were used to detect the $\left[\mathrm{Ca}^{2+}\right]_{\mathrm{i}}$ and NO production within our perfused microfluidic microvessel model, and finally the temporal and spatial changes of endothelial $\left[\mathrm{Ca}^{2+}\right]_{\mathrm{i}}$ and NO was performed with a method that was developed in individually perfused intact microvessels. Our model achieved similar results comparing with the animal model and proved to be a good parallel study platform for biological applications.

\subsection{Background}

A physiologically realistic microvessel model is able to overcome certain critical constraints of the current in vitro models, such as creating a stable and mature endothelium with appropriate barrier functions. Additionally, the advanced models, that enable a close simulation of in vivo environment with sufficient complexity, could work as artificial surrogates for curtailing high experimental costs and complexities associated with animals and in vivo studies. (El-Ali, Sorger et al. 2006) Several groups have pioneered in the development of advanced microvessel models, which applied micromanufacturing and microfluidic techniques, used either polymer or hydrogels to template the growth of vascular endothelial cells (ECs), co-cultured ECs with other vascular cells, demonstrated cell viability, and appropriate functions in angiogenesis and thrombosis. (Price, Wong et al. 2010; Tsai, Kita et al. 2012; Zheng, Chen et al. 2012; Bischel, Young et al. 2013) 
Nonetheless, prior methodologies have not been able to demonstrate and quantify some key features for microvessels, such as intracellular $\mathrm{Ca}^{2+}$ concentration $\left(\left[\mathrm{Ca}^{2+}\right]_{\mathrm{i}}\right)$ and nitric oxide (NO) production in cultured microvessels with well-developed endothelial junctions. NO, is essential for controlling vascular tone and resistance in arterioles, and regulating vascular wall adhesiveness and permeability in venules. (Xu, Zhou et al. 2013) Additionally, the endothelial calcium $\left(\left[\mathrm{Ca}^{2+}\right]_{\mathrm{i}}\right.$ ) plays an important role in microvessel permeability, (Zadeh, Glass et al. 2008; Yuan and He 2012) angiogenesis (Munaron 2006) and morphogenesis. (Lee, Thangada et al. 1999) However, in microfluidic based systems, there are very limited reports for measurements of $\left[\mathrm{Ca}^{2+}\right]_{\mathrm{i}}$ and NO production. Some studies reported DAF-2DA loaded endothelial cells from a microfluidic network (Tsai, Kita et al. 2012; Kim, Lee et al. 2013) and ATP-induced NO production was reported on cultured bovine pulmonary artery endothelial cells within a straight microfluidic microchannel. (Oblak, Root et al. 2006; Letourneau, Hernandez et al. 2010) However, none of the previous studies quantified the time-dependent changes in NO production under basal and stimulated conditions, and agonist-induced changes in $\left[\mathrm{Ca}^{2+}\right]_{\mathrm{i}}$ have not been reported in endothelialized microchannels. (Corson, James et al. 1996; Melchior and Frangos 2012)

\subsection{1 $\left[\mathrm{Ca}^{2+}\right]_{\mathrm{i}}$ and NO as key parameters to represent functional microvessel}

The activity of the endothelial cells depends heavily on changes in intracellular calcium

concentration $\left(\left[\mathrm{Ca}^{2+}\right]_{\mathrm{i}}\right)$. Resting cells have a very low concentration of cytosolic $\mathrm{Ca}^{2+}(\sim 100 \mathrm{nM})$ which is approximately 20,000-fold lower than that of extracellular $\mathrm{Ca}^{2+}$. (Carmignoto, Pasti et al. 1999) The $\left[\mathrm{Ca}^{2+}\right]_{i}$ can be increased by calcium uptake from the extracellular space via membrane calcium channels and calcium pumps (Brown and Davis 2002) or calcium release from the intracellular stores that is mostly from the sarcoplasmic reticulum and the mitochondria. (Félétou 
2011) The elevation in the endothelial $\left[\mathrm{Ca}^{2+}\right]_{\mathrm{i}}$ activates two potassium channels (Marchenko and Sage 1993) and produces the hyperpolarization of the endothelial cells. This in turn favors the entry of calcium by enhancing the corresponding electrical driving force (Luckhoff and Busse 1990) and contributes to the activation of calcium-sensitive enzymes such as eNOS. (Stankevicius, Lopez-Valverde et al. 2006; Holton, Mohamed et al. 2010) Additionally, the hyperpolarization of the endothelial cells can also evoke the endothelium-dependent hyperpolarization of the vascular smooth muscle cells. (Feletou and Vanhoutte 2006)

The regulation of the endothelial barrier function by $\left[\mathrm{Ca}^{2+}\right]_{\mathrm{i}}$ in venular microvessels has been studied in both animal models (intact microvessels) and in vitro cultured endothelial cells. Based on the current understanding, there was a hypothesis that a cascade of calcium-dependent mechanisms determines the initial increase in permeability after exposure to acute inflammatory mediators. (Michel and Curry 1999) Studies in individually perfused intact microvessels indicated that the increase in endothelial $\left[\mathrm{Ca}^{2+}\right]_{\mathrm{i}}$ induced by inflammatory mediator was rely on a passive conductance pathway. The magnitude of the initial increase for calcium entry was due to the opening of calcium activated potassium channels and the increasing of electrochemical driving force. The potassium conductance of the cell membrane was shown to play an important role in calcium influx regulation by either increase or decrease the driving force.(He and Curry 1993; He, Zhang et al. 1996) Voltage gated calcium channels did not seem to make a significant contribution to calcium influx into endothelial cells in vivo. (Curry 1992) The preference for a cascade of calcium dependent mechanism, not a direct link between $\left[\mathrm{Ca}^{2+}\right]_{\mathrm{i}}$ increase and corresponding permeability increase, was based on studies that showed the change of permeability was modified without the significant modification of the initial calcium influx. (He and Curry 1993) 
Nitric oxide (NO) is a nonpolar diatomic molecule that can freely diffuse through aqueous media, access different cellular compartments, pass the plasma membrane and activate specific signal pathways in the neighboring cells. (Lancaster and Gaston 2004) Due to its radical property, NO reacts quicker with the metal centers of heme-containing proteins than with oxygen. As a result, it shows specific signaling functions such as dampen inflammatory signaling in the endothelium via activation of cGMP/PKG. (Rivero-Vilches, de Frutos et al. 2003) Most of the existing information regarding the permeability-inducing effects of NO in microvasculature comes from the tests introducing pharmacological NOS isoform-selective inhibitors and chemical NO donors. Those inhibitors and donors such as hydrogen peroxide, histone deacetylase inhibitors, micro-RNA and statins act broadly throughout the whole cell. The laminar and oscillatory shear stress is also known to enhance the transcription of endothelial nitric oxide synthase (eNOS). Dimmeler et al. applied shear stress to human umbilical vein endothelial cells (HUVECs) in a cone and plate viscometer. (Dimmeler, Fleming et al. 1999) The phosphorylation of Akt on eNOS protein was detected by western blot analysis with phosphospecific Akt antibodies. It is possible to measure the phosphorylation of eNOS or eNOS mRNA as the half-life of those bio-products is relatively long (10 to 35 hours). However, such measurement could only partially predict overall enzymatic activity as eNOS was subjected to several overlapping regulatory modifications. For example eNOS, which was the preponderant NOS isoform in healthy endothelial cells, was activated by receptor-dependent/independent agonists (such as ATP) as a consequence of an increase in the endothelial $\left[\mathrm{Ca}^{2+}\right]_{\mathrm{i}}$ and the association of caveolin-1 (Cav-1) and $\mathrm{Ca}^{2+} / \mathrm{Ca}^{2+}$-calmodulin (CaM) complex. (Fleming and Busse 1999) Caveolin-1 (Cav-1) was a plasma membrane associated scaffolding protein that could inhibit eNOS activity by direct sequestration that limited the access of binding site from calcium- 
calmodulin. (Bernatchez, Sharma et al. 2011) $\mathrm{Ca}^{2+}$-calmodulin (CaM), on the other hand, was one of the most intensively studied intracellular binding proteins that converted the $\mathrm{Ca}^{2+}$ signals into different biochemical changes. (Chin and Means 2000) The identifying of CaM-binding domain in the primary structure of eNOS (Bredt, Hwang et al. 1991; Marsden, Schappert et al. 1992) and the finding of CaM binding proteins that inhibit enzyme activity (Fleming and Busse 2003) showed that the binding of a $\mathrm{Ca}^{2+} / \mathrm{CaM}$ complex was essential to activate the constitutive enzyme. Figure 7.1 shows the regulation function of Cav-1 and CaM on eNOS in endothelium. eNOS that was bound to Cav-1 on the inner leaflet of the plasma membrane was inactive. (Figure 7.2a) The eNOS was activated by $\mathrm{Ca}^{2+} / \mathrm{CaM}$ by replacing itself with $\mathrm{Cav}-1$ at the same binding site. After the application of tetrahydrobiopterin $\left(\mathrm{BH}_{4}\right)$ as a cofactor, eNOS performed the NO synthases function by the conversion of $\left[{ }^{3} \mathrm{H}\right] \mathrm{L}$-arginine to $\left[{ }^{3} \mathrm{H}\right] \mathrm{L}$-citrulline. (Lee, Robinson et al. 1995) (Figure 7.1b)
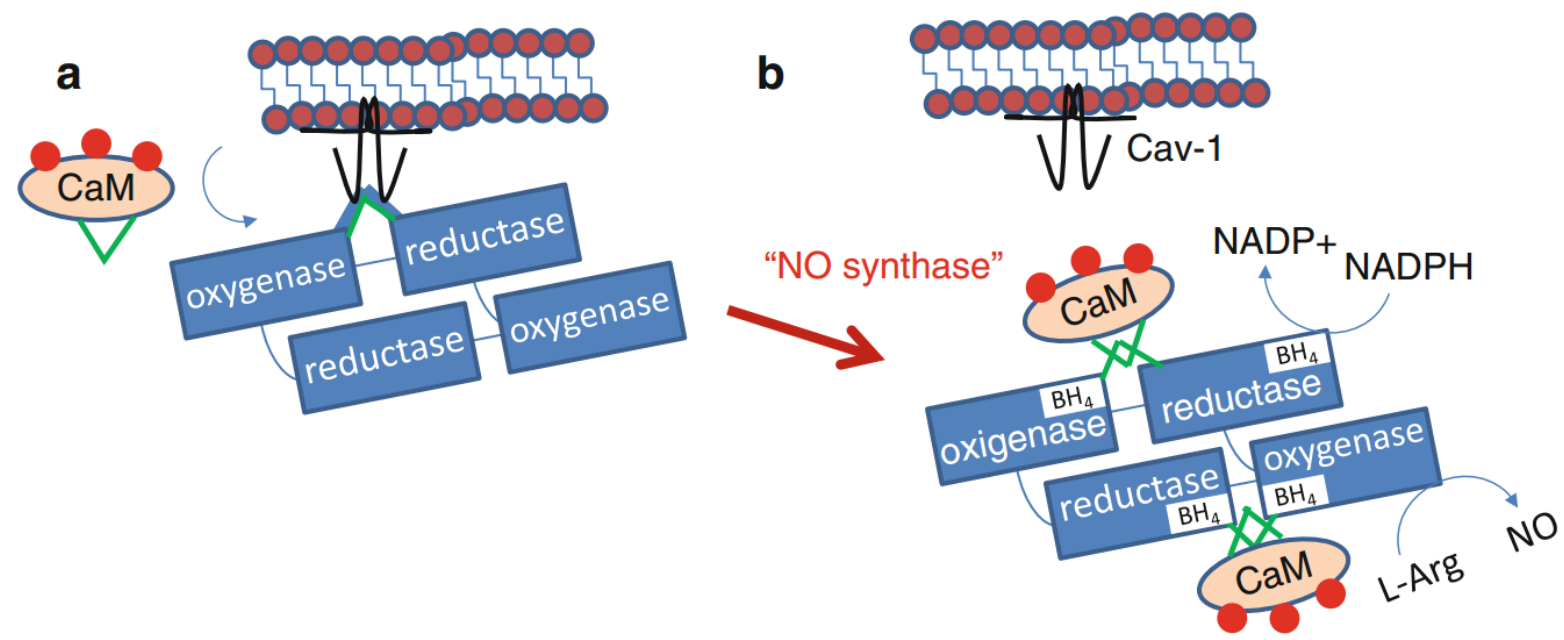

Figure 7.1 The regulation function of Cav-1 and CaM on eNOS in endothelium. a) eNOS that bound to caveolin-1 (Cav-1) on the inner leaflet of the plasma membrane was inactive. b) Dimerized eNOS became activated by $\mathrm{Ca}^{2+} / \mathrm{CaM}$ that was bound to the same site as Cav-1. eNOS worked as an NO synthase when cofactor $\mathrm{BH}_{4}$ was in supply. (Bonini, Dull et al. 2014) 
The corresponding NO production has been shown to play important roles in the regulation of microvessel permeability. For example the endothelial gap formation in response to agonists induced NO production was present in animal model (Zhu and He 2005; Zhou and He 2010; Zhou and He 2011) as well as in vitro cell culture model.(Wysolmerski and Lagunoff 1990; Yamamoto, Furuya et al. 2011)

\subsubsection{Endothelial $\left[\mathrm{Ca}^{2+}\right]_{i}$ and NO stimulated by ATP}

In our study, we chose ATP as the representative agonist to study receptor-mediated changes

in endothelial $\left[\mathrm{Ca}^{2+}\right]_{\mathrm{i}}$ and NO production. ATP was released by different cell types such as red blood cells or aggregated platelets. The release was also a response to mechanical or biochemical stimulation such as tissue injury or under inflammatory conditions.

ATP was not only the source of the energy that drives virtually all cell functions, but also functioned as an autocrine and paracrine regulatory signaling molecule. The modulation of cell function by ATP was primarily via activating nearby purinoceptors, such as ion channel P2X receptors and G-protein-coupled P2Y receptors that was expressed on most types of endothelial cells. (Carter, Hallam et al. 1988; Dubyak and el-Moatassim 1993; Yegutkin 2008) The increased levels of ATP caused the release of endothelial-derived relaxing factor and triggered the synthesis of prostacyclin, and increases in microvessel permeability by increasing endothelial $\left[\mathrm{Ca}^{2+}\right]_{i}$. (Mannix, Moatter et al. 1993; He, Zhang et al. 1996; Sprague, Olearczyk et al. 2003)

\subsubsection{Fluorescent probes for $\left[\mathrm{Ca}^{2+}\right]_{\mathrm{i}}$ and NO detection}

Fluo-4 was one of the high affinity $\mathrm{Ca}^{2+}$ indicators that have already been well characterized with a sufficient array of spectral properties and binding affinities. It was a single wavelength dye and could be excited by argon laser $(488 \mathrm{~nm})$ from confocal microscopy, flow cytometry or fluorescein filter sets in wide-field epi-fluorescent microscopes. Fluo-4 was brighter and more 
photostable than the most commonly used Fluo-3. There were other advantages such as low background absorbance, lower dye concentration requirement, and shorter incubation time. (Paredes, Etzler et al. 2008) At rest and in the $\mathrm{Ca}^{2+}$ free form, fluorescence was minimal. However, its fluorescence increased over 100 -fold when it bond with $\mathrm{Ca}^{2+}$. The chemical structures of fluo-4 and related compounds are shown in Figure 7.2. (Gee, Brown et al. 2000)
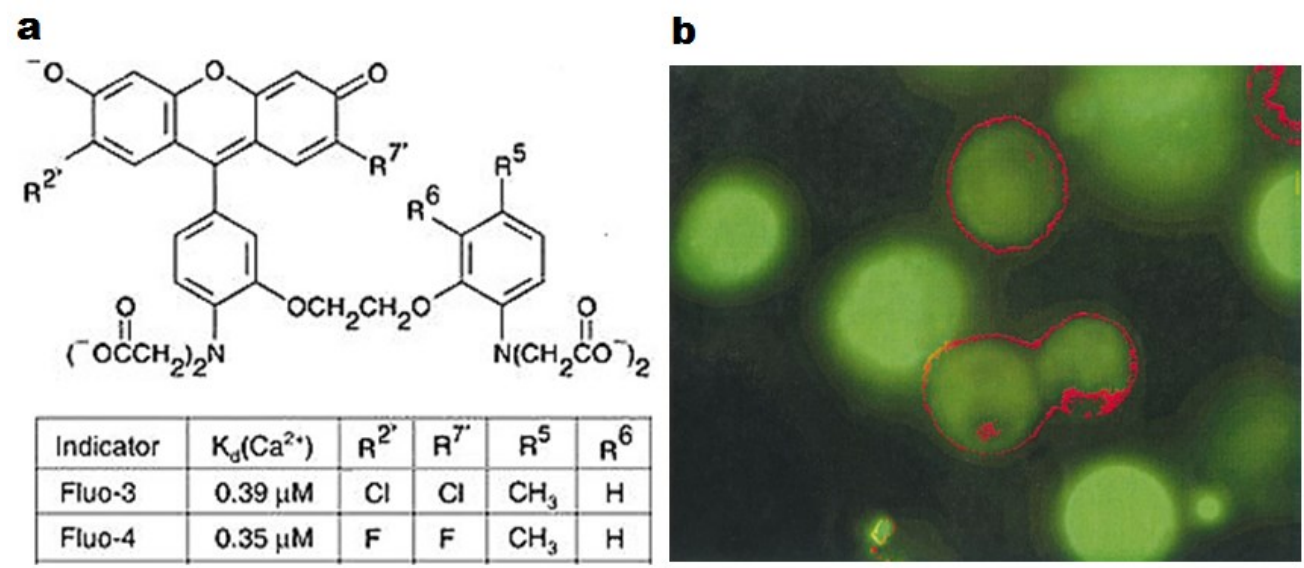

Figure 7.2 a) The chemical structures of fluo-3 and fluo-4. b) Double label imaging shows that fluo-4-labeled cells were brighter than fluo-3-labeled cells. The green label from double-labeled cells (fluo-3-filled with red halo) was much dimmer than the green label in fluo-4-filled cells. (Gee, Brown et al. 2000)

DAF-2 was one of the nitric sensitive fluorescent probes by utilizing the reaction of NO with $o$-phenylenedi-amines to produce corresponding benzotriazoles, as shown in Figure 7.3. The high value of highest occupied molecular orbital (HOMO) energy of phenylenediamines resulted in efficient quenching of adjacent fluorophores by photoinduced electron transfer (PeT). Moreover, the low HOMO energy of benzotriazoles induced a highly fluorescent binding between the adjacent fluorophores and NO. (Kobayashi, Ogawa et al. 2010) As a result, DAF-2 showed excellent selection and sensitivity, as it had weak fluorescence before reaction with NO, and became highly fluorescent after the reaction. (Nagano 2010) 


\section{a}

Key reaction: Benzotriazole formation

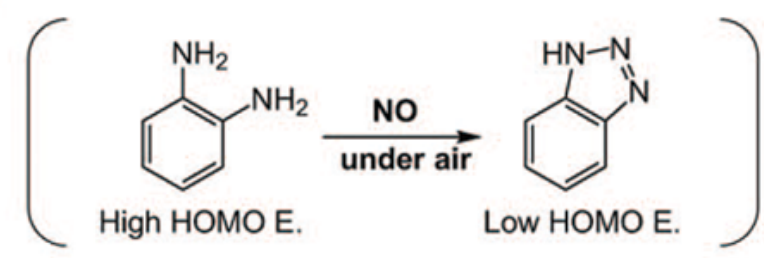

b
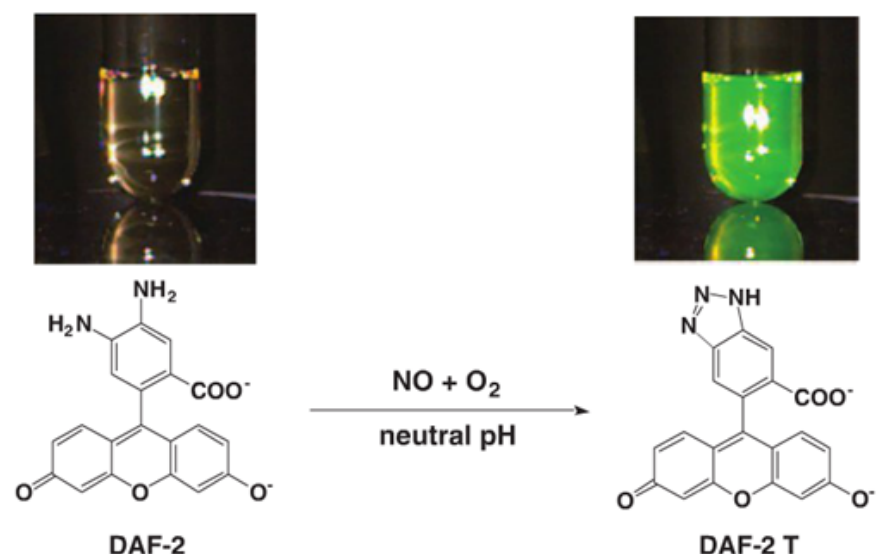

\section{DAF-2}

Ex. $\max : 486 \mathrm{~nm}$ Em. $\max : 520 \mathrm{~nm}$

$\Phi_{n}: 0.004$

Ex. $\max : 491 \mathrm{~nm}$ Em. $\max : 513 \mathrm{~nm}$

$\Phi_{n}: 0.92$

Figure 7.3 Schemes for the fluorescence signal activation of fluorescein-based probes reacted with NO. a) Key reaction of benzotriazole formation. (Kobayashi, Ogawa et al. 2010) b) DAF-2 was converted to a triazole compound DAF-2 $\mathrm{T}$ and caused slightly change of maximum absorbance but significant change in fluorescence intensity. (Nagano 2010)

Even though DAF did not react with NO directly, but with an active intermediate $\mathrm{NO}^{+}$or its equivalent species, it was still safe to use DAF-2 as an NO probe under anaerobic conditions when NO was easily oxidized. It was usually necessary to prepare the diacetate DAF-2 DA that could permeate well into the cells. DAF-2 DA was hydrolyzed to yield DAF-2 by esterase in the cytosol, which was retained in the cell owing to its relatively poor permeating ability. (Kojima, Nakatsubo et al. 1998) However, it has been reported there was increased DAF-2 leakage from endothelial cells with the increase of microvessel permeability. To overcome this issue and perform valid NO measurement, a continuous DAF-DA loading was performed during the NO 
measurement to achieve a relatively constant DAF-2 concentration in cultured endothelial cells. (Zhou and He 2011)

\subsection{Method}

\subsubsection{Confocal fluorescence imaging of $\left[\mathrm{Ca}^{2+}\right]_{\mathrm{i}}$}

The image acquisition for endothelial $\left[\mathrm{Ca}^{2+}\right]_{\mathrm{i}}$ detection induced by ATP was conducted on a Leica TCS SL confocal microscope with a Leica $\times 25$ objective (NA: 0.95 ). Based on the fluorescent probes we chose (fluo-4), an argon laser $(488 \mathrm{~nm})$ at $50 \%$ power was used for excitation, and the emission band was 510-530 nm. Figure 7.4 shows the schematic procedures.

After HUVECs reached confluence and successfully covered the entire inner surface of the network microchannel, each device was first perfused with albumin-Ringer solution by either manually loading with gravitational force or perfusion with syringe pump multiple times to remove the entire medium residue. (Figure 7.4a) Then the microvessel model was perfused with fluo-4 AM (5 $\mu \mathrm{M}$, diluted with albumin-Ringer solution) within the incubator for 40 minutes. (Figure 7.4b) The perfusion rate was the same as long-term perfusion culture. Albumin-Ringer solution was prepared by adding 1\% BSA into Ringer solution. The composition of the mammalian Ringer solution was (in $\mathrm{mM}$ ) $132 \mathrm{NaCl}, 4.6 \mathrm{KCl}, 2 \mathrm{CaCl} 2,1.2 \mathrm{MgSO} 4,5.5$ glucose, 5.0 NaHCO3, 20 N-2-hydroxyethylpiperazine-N'-2-ethanesulfonic acid (HEPES) and Na-HEPES. The added BSA was to prevent cell starvation. After the loading, the model was again perfused with albumin-Ringer to rinse the lumen fluo-4 AM. The microvessel model was then transferred onto the confocal microscopy stage. (Figure 7.4c) 


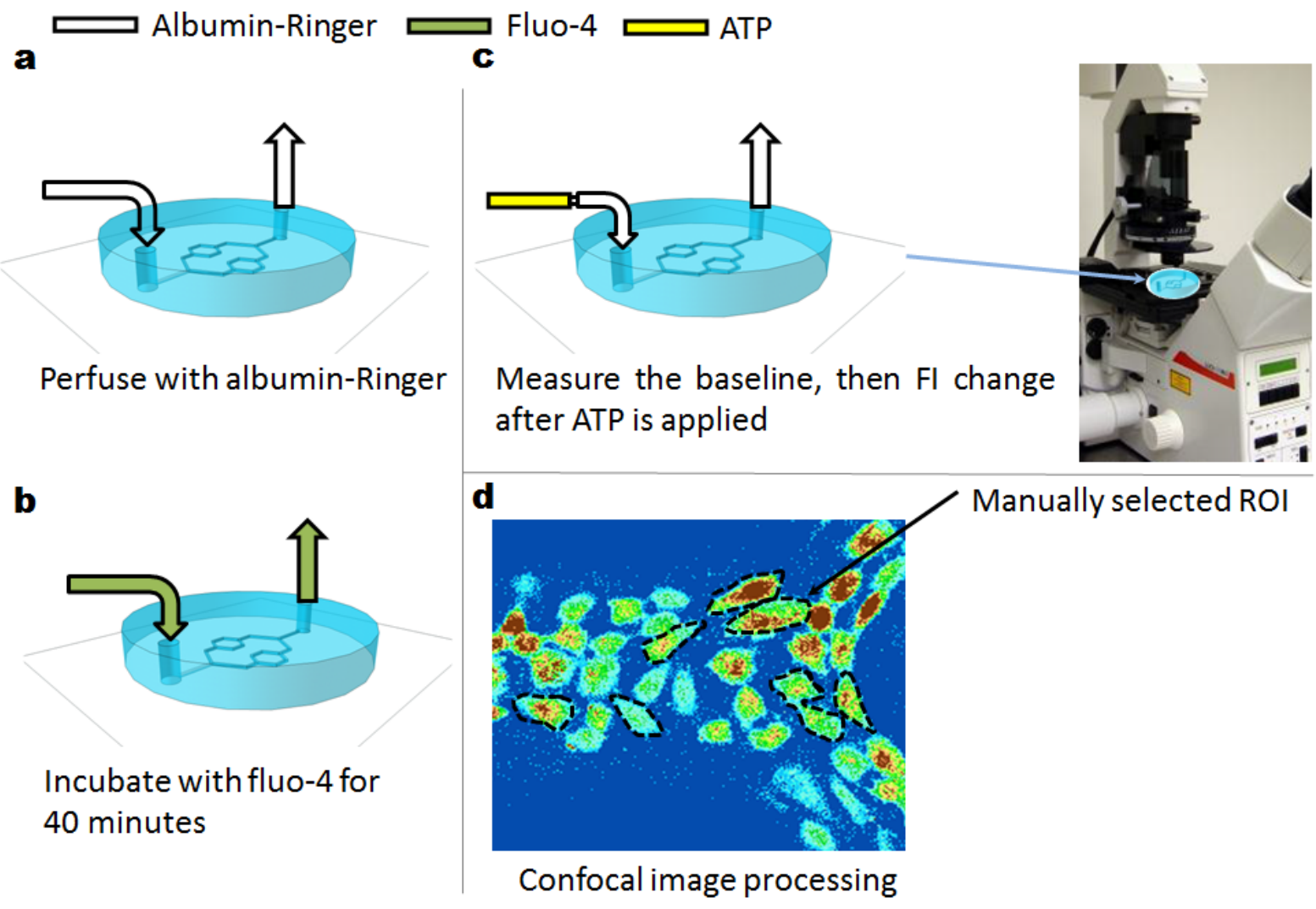

Figure 7.4 The schematic procedures for endothelial $\left[\mathrm{Ca}^{2+}\right]_{\mathrm{i}}$ detection induced by ATP. a) After endothelial cells reached confluence, the device was perfused with albumin-Ringer solution. b) Fluo-4 was perfused into the device for 40 minutes within the incubator. c) The device was first perfused with albumin-Ringer for baseline measurement, then $10 \mu \mathrm{M}$ ATP to detect the $\left[\mathrm{Ca}^{2+}\right]_{\mathrm{i}}$ response. d) The confocal image was stacked with maximum intensity projection, and each area of individual endothelial cells was manually selected as ROI, and processed by Metafluor software (Universal Imaging).

The baseline was measured by 10 minutes perfusion of plain albumin-Ringer solution. After $10 \mu \mathrm{M}$ of ATP was perfused into the device, the fluorescence intensity (FI) change was collected by the confocal system. To minimize the effect of photobleaching, fluo-4 images were collected using a $512 \times 512$ scan format at a z-step of $2 \mu \mathrm{m}$. The measurement of the baseline was taken every 2 minutes for a total of 10 minutes and the whole FI change in response to ATP was taken every 20 seconds. The stack number of confocal imaging, the focusing, and the testing region 
were kept the same all through the whole experiment. Quantitative analysis of endothelial $\left[\mathrm{Ca}^{2+}\right]_{\mathrm{i}}$ at the individual endothelial cell level was conducted using manually selected ROIs along the microchannels as shown in Figure 4d. Each ROI covered the area of one individual endothelial cell, as indicated by the fluorescence outline. Endothelial $\left[\mathrm{Ca}^{2+}\right]_{\mathrm{i}}$ at the cellular level were quantified by calculating the mean fluorescence intensity (FI) of each stack of ROIs after the subtraction of the background auto fluorescence. The percent change in FI was expressed as $\mathrm{FI} / \mathrm{FI}_{0} * 100$, where $\mathrm{FI}_{0}$ was the initial baseline $\mathrm{FI}$ of fluo-4. All the images were analyzed with commercial software Metafluor (Universal Imaging).

\subsubsection{Fluorescence imaging of NO production}

A similar approach was conducted to investigate the endothelial NO at the cellular levels in the microvessel network using DAF-2 DA and fluorescence imaging.(Figure 7.5) Experiments were performed on a Nikon Diaphod 300 microscope equipped with a 12-bit digital CCD camera (ORCA; Hamamatsu) and a computer controlled 100 shutter (Lambda 10-2; Sutter Instrument; Novato, CA). The light source was a 75-W xenon lamp. The excitation wavelength for DAF-2 was selected by an interference filter $(480 / 40 \mathrm{~nm})$, and emission was separated by a dichroic mirror $(505 \mathrm{~nm})$ and a band-pass barrier $(535 / 50 \mathrm{nM})$.

After long-term perfusion culture, the device was first rinsed with albumin-Ringer solution, and then incubated with perfused DAF-2 DA $(5 \mu \mathrm{M})$ for $35 \sim 40$ minutes. Throughout the whole experiment, the DAF-2 DA was always present in the perfusate to overcome DAF-2 leakage. The fluorescent images were collected from a fixed testing region with a fixed focal plane using a Nikon Fluor lens (x20, NA: 0.75). 
After the microvessel model was placed onto the microscope, plain DAF-2 solution was perfused to measure the steady state of mean FI which indicated basal NO production, and followed by $10 \mu \mathrm{M}$ ATP to detect corresponding NO production. Finally sodium nitroprusside (SNP, 50mM), an NO donor, was perfused into the microvessel model to verify the sufficiency of DAF-2 in the cells and the responsiveness of DAF-2 to NO.

Data analysis was conducted at the individual endothelial cell level using manually selected regions of interests (ROIs). (Figure 7.5d) Each ROI covered the area of one individual cell as indicated by the fluorescence outline upon the introduction of SNP.

The auto fluorescence of PDMS from the surrounding area outside of microchannel was subtracted from all of the measured FIs. Because the fluorescent chemical transformation of DAF-2 to DAF-2T is irreversible, the detected DAF-2 FI reflected the cumulated total amount NO production.(Yi, Zhang et al. 2002) As a result, the direct measurement of continuous NO production based on DAF-2 fluorescence curve does not represent the actual NO concentration.

To accurately present the relationship between NO production and the stimulation of ATP, first differential conversion of the time dependent DAF-2 FI (solid line in Figure 7.7) was used to present the NO production rate (df/dt, as dash-dotted line in Figure 7.7). (Zhu and He 2005) The changes in $\mathrm{FI}_{\mathrm{DAF}}$ upon ATP stimulation were expressed as the net changes in FI $(\Delta \mathrm{FI})$. FI was expressed in arbitrary units (AU) measured with identical instrumental settings. The whole experiment for one device last around 40 minutes. 


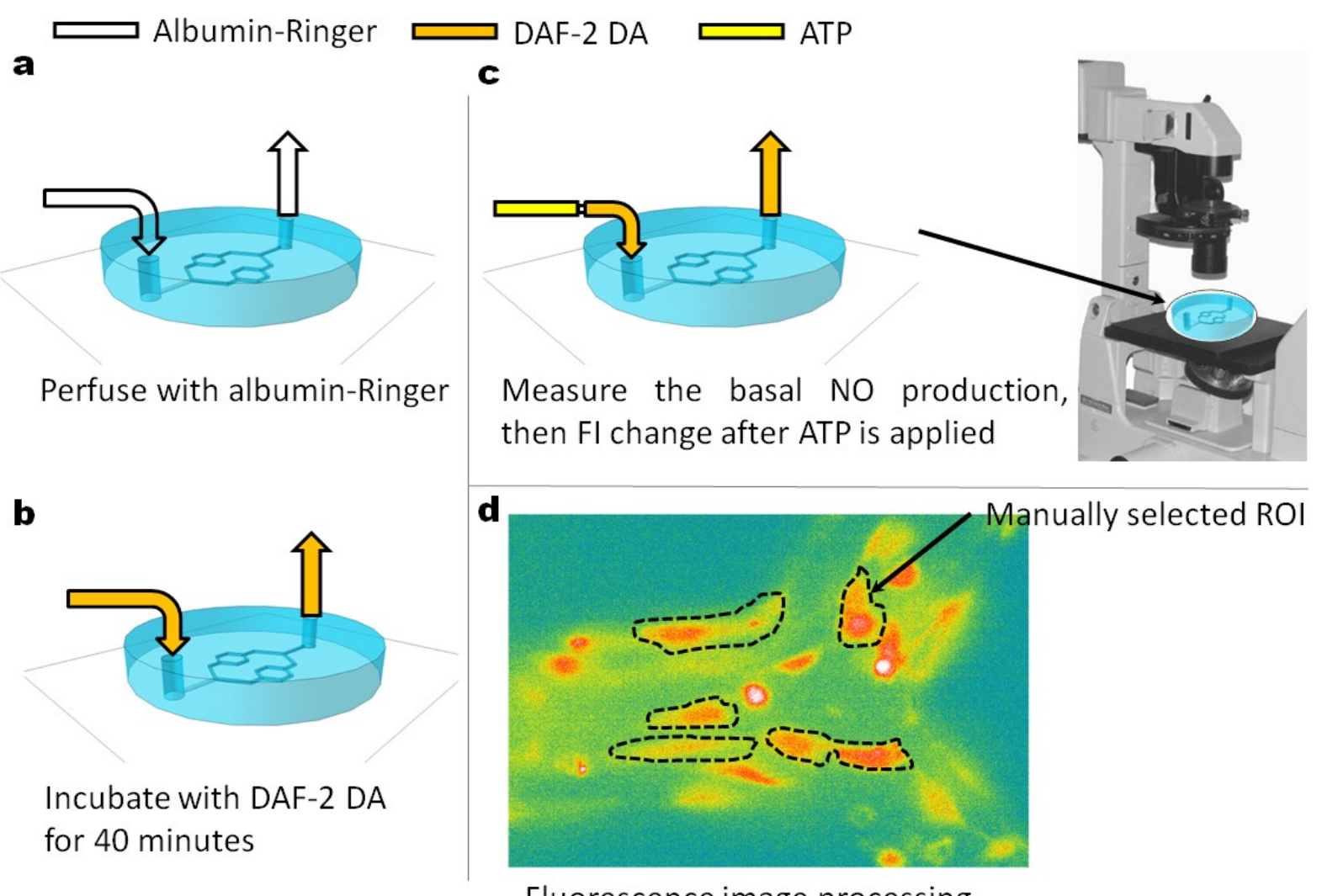

Fluorescence image processing

Figure 7.5 The schematic procedures for NO detection. a) Device was rinsed with albuminRinger. b) DAF-2 DA was perfused for 40 minutes within the incubator. c) The basal NO production and FI change were measured with the microscopic system. d) After manually select each ROI, the image data was processed to calculate the changes in $\mathrm{FI}_{\mathrm{DAF}}$.

All values are means $\pm \mathrm{SE}$. Each experiment was performed on one testing region from one device, and $\mathrm{n}$ value presents the number of device that was used for the experiments.

\subsection{Result and discussion}

\subsubsection{Endothelial $\left[\mathrm{Ca}^{2+}\right]_{i}$ change induced by ATP}

Increases in endothelial $\left[\mathrm{Ca}^{2+}\right]_{i}$ have been demonstrated to play important roles in the regulation of a variety of microvessel functions including endothelial barrier function, i.e. microvessel permeability. In individually perfused microvessels, inflammatory mediator commonly induces transient increases in endothelial $\left[\mathrm{Ca}^{2+}\right]_{i}$ followed by transient increases in 
microvessel permeability. To validate the biological functions of the microvessel model developed within our microfluidic microchannel network, we applied the method developed in individually perfused intact microvessels (He, Pagakis et al. 1990; Zhou and He 2011) and quantitatively measured the changes in endothelial $\left[\mathrm{Ca}^{2+}\right]_{\mathrm{i}}$ after the endothelial cells was exposed to ATP. (Figure 7.6) Experiments were conducted in four microvessel models with fluo-4. Fluo4 is a single wavelength dye with high fluorescence thus suitable for confocal imaging.

a

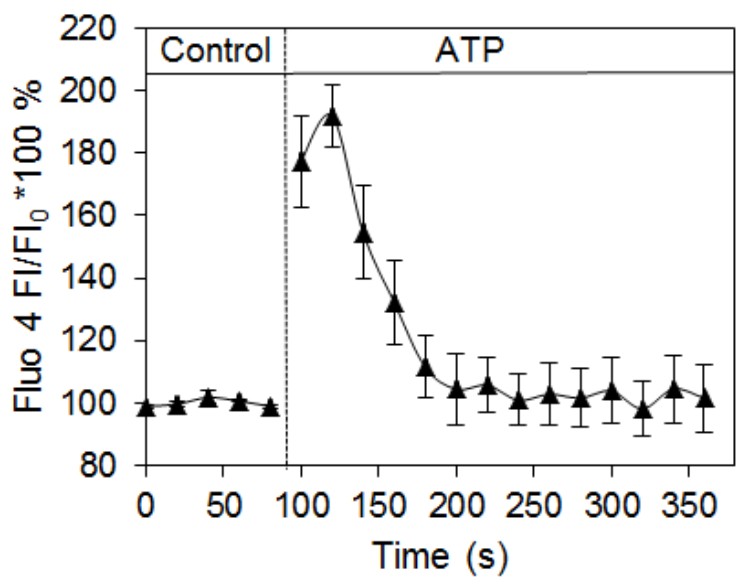

b
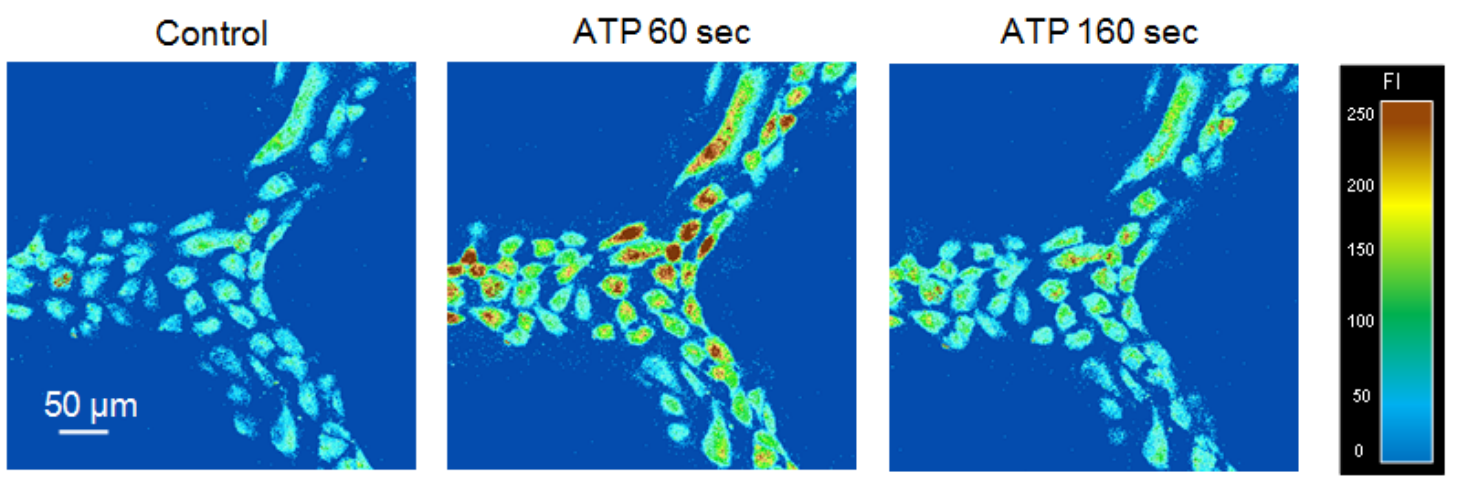

Figure 7.6 ATP-induced endothelial cell $\left[\mathrm{Ca}^{2+}\right]_{i}$ increase in HUVECs cultured microchannel network. a) Time course of ATP-induced changes in endothelial $\left[\mathrm{Ca}^{2+}\right]_{\mathrm{i}}$ from a representative experiment. b) Fluo-4 confocal images from one representative experiment before and after the start of ATP perfusion at 60 and 160 seconds. 
After 10 minutes of perfusion of albumin-Ringer to measure the baseline as control, ATP $(10 \mu \mathrm{M})$ was perfused into each device. The corresponding $\left[\mathrm{Ca}^{2+}\right]_{\mathrm{i}}$ response in all endothelial cells was quantified by calculating the mean FI of each stacks of confocal images after subtracting the background autofluorescence. As shown in Figure 7.6a, the FI reached a peak at $35 \pm 10 \mathrm{sec}$ with the mean peak $\left[\mathrm{Ca}^{2+}\right]_{\mathrm{i}}$ at $187 \pm 22 \%$ of the baseline. Representative fluorescence images of the control (baseline), 60 seconds, and 160 seconds of the testing region in microvessel model are shown in Figure 7.6b respectively.

\subsubsection{Measurement of ATP induced NO production}

In intact venules, it has been demonstrated that inflammatory mediator-induced increases in endothelial $\left[\mathrm{Ca}^{2+}\right]_{\mathrm{i}}$ was associated with increased NO production and both increased endothelial $\left[\mathrm{Ca}^{2+}\right]_{\mathrm{i}}$ and NO production contribute to increases in microvessel permeability (Zhu and He 2005; Zhou and He 2011). With demonstrated ATP-induced increases in endothelial $\left[\mathrm{Ca}^{2+}\right]_{\mathrm{i}}$, we further measured NO production in response to ATP in DAF-2 loaded endothelial cells lining the microchannels. Figure 7.7 shows the quantification of time-dependent changes in $\mathrm{FI}_{\mathrm{DAF}}$ and correlated images from an individual experiment. When ATP $(10 \mu \mathrm{M})$ was added to the rate change in ATP-induced NO production was measured with continuous DAF-2 DA perfusion (Zhou and He 2011). Each device was first perfused with albumin-Ringer solution containing DAF-2 DA $(5 \mu \mathrm{M})$ for $35 \sim 40$ minutes before collecting images. The basal NO production rate was calculated from the slope of the mean FI increase during albumin-Ringer perfusion after DAF-2 loading reached the steady state. The rate change was $0.15 \pm 0.05$ AU per min, which was very close to the basal NO production rate measured in intact rat mesenteric venules: $0.11 \pm$ $0.006 \mathrm{AU}$ per min,(Zhou and He 2011) $0.13 \pm 0.05 \mathrm{AU}$ per min,(Xu, Zhou et al. 2013) and $0.1 \pm$ $0.01 \mathrm{AU} / \min ($ Zhou, Yuan et al. 2013). 


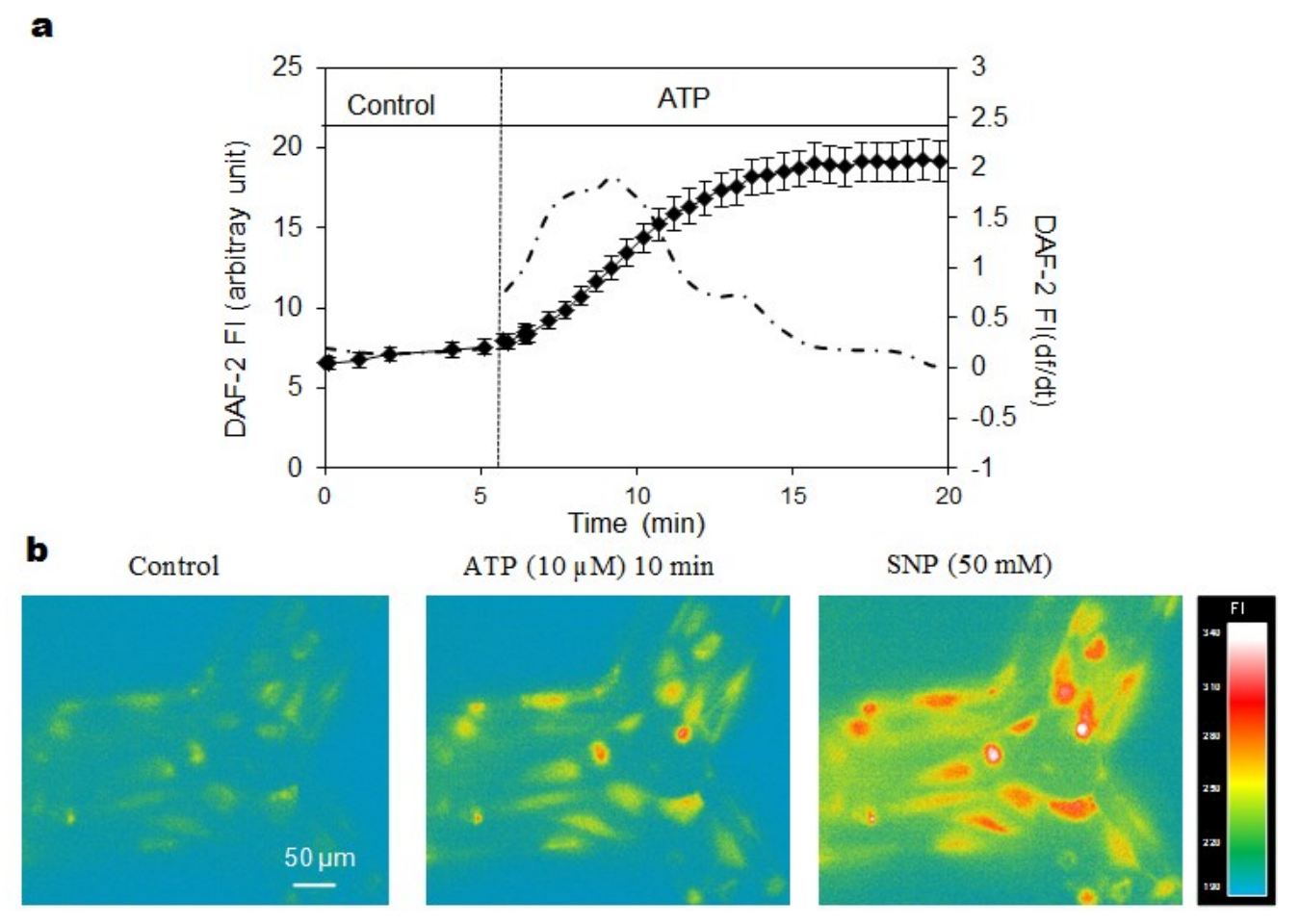

Figure 7.7 ATP-induced NO production increases in HUVECs cultured microchannel network. a) Time dependent changes in $\mathrm{FI}_{\mathrm{DAF}}$ (left $\mathrm{Y}$ axis) from a representative experiment before and after ATP $(10 \mu \mathrm{M})$ stimulation with continuous perfusion of DAF-2 DA at $5 \mu \mathrm{M}$. The first differential conversion of cumulative $\mathrm{FI}_{\mathrm{DAF}}$ (df /dt, right $\mathrm{Y}$ axis) represents the NO production rate (dashdotted line). b) Representative DAF-2 fluorescence images. ATP induced significant increases in NO-DAF-2 fluorescence intensity (FI) relative to control (left). FI was further increased with the application of the NO donor sodium nitroprusside (SNP).

When ATP $(10 \mu \mathrm{M})$ was added to the perfusate, FI $_{\text {DAF }}$ was significantly increased. The rate of $\mathrm{FI}_{\mathrm{DAF}}$ change was derived by first differential conversion of cumulative $\mathrm{FI}_{\mathrm{DAF}}$ over time. The peak rate of $\mathrm{FI}_{\mathrm{DAF}}$ increase was $1.18 \pm 0.37 \mathrm{AU}$ per min. The increased $\mathrm{FI}_{\mathrm{DAF}}$ returned to the steady state level after 10 15 minutes of ATP exposure. Data were derived from 3 devices with a total of 35 ROIs and 11 to 12 ROIs per microvessel model. The NO production rate change before and after ATP stimulation was indicated as dash-dotted line. Figure 7.7b shows the fluorescent image during the steady state of the microvessel model, the increased FI at 10 min of 
ATP exposure and much further increased FI after SNP was introduced into the model, respectively.

\subsubsection{Discussion of ATP induced $\left[\mathrm{Ca}^{2+}\right]_{i}$ change and NO production}

The major technical improvement for biology and physiology application reported on this model is the quantitative measurements of agonist-induced changes in endothelial $\left[\mathrm{Ca}^{2+}\right]_{\mathrm{i}}$ and NO production at individual cell levels. Studies in individually perfused intact microvessels indicated that inflammatory mediator-induced increases in endothelial $\left[\mathrm{Ca}^{2+}\right]_{\mathrm{i}}$ is essential for increases in microvessel permeability and that the magnitude of the $\left[\mathrm{Ca}^{2+}\right]_{i}$ determines the degree of permeability increases.(He, Pagakis et al. 1990; He and Curry 1991; Curry 1992; He and Curry 1994; He, Zhang et al. 1996) In addition, agonist-induced $\mathrm{Ca}^{2+} /$ calmodulin-dependent endothelial nitric oxide (NO) synthase (eNOS) activation and NO production have been shown to play important roles in the regulation of microvessel permeability.(Zhu and He 2005; Zhou and He 2010; Zhou and He 2011) In this study, we directly applied the methods developed in individually perfused intact microvessels(He, Pagakis et al. 1990; Zhou and He 2011) to this in vitro microvessel model as parallel studies to those performed in the intact microvessels in vivo.

The application of microfluidics to study intracellular calcium (Kou, Pan et al. 2011; Xu, Yue et al. 2013) and NO responses to agonist have been reported by a few investigators. However, the calcium studies were either from suspended leukemic cells (Xu, Yue et al. 2013) or from non-confluent osteoblasts. (Kou, Pan et al. 2011) Though ATP-induced NO has been reported in bPAEC cultured microfluidic device, there was no characterization of the junctions of endothelial cells and no temple resolution of the NO production was present in that study. (Oblak, Root et al. 2006) In our model, we choose ATP as the representative agonist to study receptormediated changes in endothelial $\left[\mathrm{Ca}^{2+}\right]_{\mathrm{i}}$ and NO production. ATP can be released by red blood 
cells, aggregated platelets, and injured tissue or under inflammatory conditions. The increased levels of ATP cause the release of endothelial-derived relaxing factor and trigger the synthesis of prostacyclin, and increase in microvessel permeability by increasing endothelial $\left[\mathrm{Ca}^{2+}\right]_{\mathrm{i}}$. (Mannix, Moatter et al. 1993; He, Zhang et al. 1996; Sprague, Olearczyk et al. 2003) The action of ATP is primarily via the purinergic P2Y receptor expressed on most types of endothelial cells. (Carter, Hallam et al. 1988; Dubyak and el-Moatassim 1993) The calcium measurements using fluo-4 as indicator in the microvessel network showed similar responses to what we found in intact microvessels. (He, Zhang et al. 1996; Zhou and He 2011)

As for NO measurements using DAF-2 DA, we need to recognize the specific manner of DAF-2 chemical conversion in the presence of NO and conduct data analysis accordingly. (Kojima, Nakatsubo et al. 1998; Zhou and He 2011) DAF-2 DA is membrane permeable and diffuses freely into the cells driven by concentration gradient and is then hydrolyzed by cytosolic esterase to form DAF-2. Intracellular DAF-2 is less membrane permeable due to its polarity, and is designed to be trapped inside the cells. Because the fluorescence chemical transformation of DAF-2 by NO is irreversible, (Kojima, Nakatsubo et al. 1998) the detected NO-sensitive fluorescence with DAF-2 represents a cumulative production of NO. The slope of the $\mathrm{FI}_{\mathrm{DAF}}$ curve represents the NO production rate and the plateau indicates the termination of $\mathrm{NO}$ production instead of the actual NO concentration in the cells. To present the real-time course of NO production, the NO production rate was quantified based on the differential conversion of that regression equation $(\mathrm{df} / \mathrm{dt})$.

Using this method, our study presented the basal NO production rate and the changes in NO production after exposure to ATP in HUVECs-developed microvessels in the microfluidic device with temporal and spacial resolution for the first time. The results are comparable to those 
derived from individually perfused intact venules. These studies provide an in vivo validation of the functional aspect of engineered microvessel network, which is instrumental for the utility of this device to the development of biological applications. 


\section{Chapter 8 Conclusions and Future Work}

\subsection{Summary of major contributions}

In this dissertation, a microvessel model was developed to mimic the geometries of microvessel networks in vivo and validated certain important features that were very important to represent the physiological and pathological condition of the microvasculature. Combing the photolithography and soft lithography techniques, two different kinds of microchannel networks were fabricated: one based on standard microfluidic fabrication method with a very thin substrate that was suitable for high-resolution confocal microscopy, and the other with multi-diameter channels with circular cross-section that obeyed the Murray's law. Endothelial cells were seeded into the microfluidic devices, and after 4 days up to 2 weeks' long-term perfusion, the cells reached confluence, and covered the inner surface of the entire channel. To characterize the microvessel model, immunofluorescence staining was peformed to examine the formation of the confluence cell monolayer and the endothelial adherens junction. Furthermore, the real-time change of endothelial $\left[\mathrm{Ca}^{2+}\right]_{\mathrm{i}}$ and NO stimulated by ATP with temporal and spacial resolution was detected and reported in the microfluidic microchannel networks for the first time. These results were comparable to what was found in individually perfused intact microvessels. The research work presented in this dissertation was important to address the critical limitations of in vitro cell culture models such as lacking a physiologically relevant fluid flow, and a confluence 3D endothelia monolayer with appropriate barrier functions. It showed the unique advantage of microfabrication and microfluidic technologies to create a cost efficient, easy to operate and reproducible microvessel model. The model recapitulated the cellular, physical, and hemodynamic environment of microvessels and allowed for high quality and real-time imaging over extended periods of time, such characteristics that have not been considered in conventional 
models were observable. The research was able to provide an improved model for comprehensive and quantitative studies of blood-endothelium interactions.

\subsection{Future work}

One of the main roles of red blood cells is to deliver oxygen to the surrounding tissues within the microcirculation. However, it is also known that the deformation of red blood cells under shear stress also releases micromolar amount of ATP, particularly in microvessels and capillaries.

The deformation of the red blood cells depended on shear stress, the effective fluid viscosity, and the boundary condition of the inner surface of the microvessel and the released ATP participated in the regulation of vascular perfusion. For example, ATP caused endotheliumdependent vasorelaxation was observed in many tissues. (Burnstock and Kennedy 1986; McCullough, Collins et al. 1997) When ATP was applied to the lumen of arterioles and venules in animal model, there was an NO dependent vasodilator response resulting in a localized increase in perfusion. (Dietrich, Horiuchi et al. 2009)

There has been microfluidic approach to study the time-dependent dynamics of deformation induced ATP release by pumping a mixture of red blood cells and luciferase/luciferin solution through a microfuidic constriction. (Wan, Ristenpart et al. 2008) Another microfluidic device was fabricated by placing a polycarbonate membrane on top of PDMS microchannels. Bovine pulmonary artery endothelial cells were seeded onto the membrane, and red blood cells were perfused through the microchannel underneath the membrane. The uptake of the ATP by the endothelial cells was characterized with DAF-FM DA. (Letourneau, Hernandez et al. 2010) This model was able to detect short-lived molecule signal such as ATP and NO, and provide a 
platform to study the interaction between different cell types within a partially flow condition. However, the endothelial cells was seeded onto a 2D flat substrate, and incubated under static condition for 2 hours. It requires at least 16 hours for endothelial cells to form adherens junction, and the lack of shear flow would affect endothelial cell biology that has been discussed in the previous chapter. (Chien, Li et al. 1998; Nerem, Alexander et al. 1998; Li, Haga et al. 2005; Chau, Doran et al. 2009; Lee, Vunjak-Novakovic et al. 2009; Lee and Niklason 2010; Potter, Lundberg et al. 2011)

A new microvessel model was designed to study the dynamics of ATP release from red blood cells under the stimulation of shear stresses, as well as the induced microvessel permeability change. For example, by applying shear flow on red blood cells within endothelialized microchannels and measuring the stimulated endothelial $\left[\mathrm{Ca}^{2+}\right]_{i}$ production, it was possible to study the participation of the released ATP in vascular signaling and circular regulation.

With the advantage of microfluidic fabrication technique, it was possible to design specific microchannels to recapitulate the shear flow and wall shear stress inducing ATP release from red blood cells. Figure 8.1a and c shows the microchannel designs for red cell deformation, and a "clotted" microvessel model respectively. The numerical simulation was able to calculate the shear rate distribution inside the channels as shown in Figure $7.1 \mathrm{~b}$ and $\mathrm{d}$. The simulated shear rate was comparable to the previous study. (Wan, Ristenpart et al. 2008) 
a

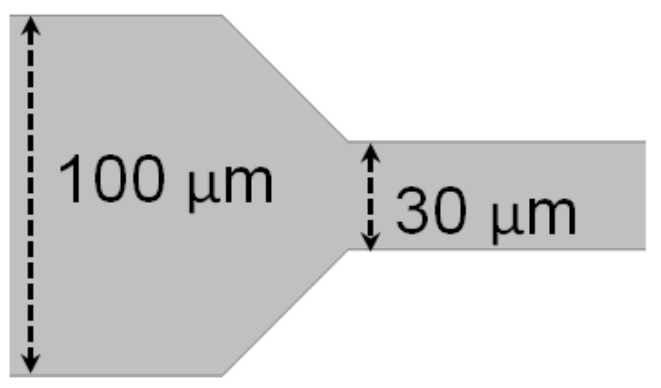

c

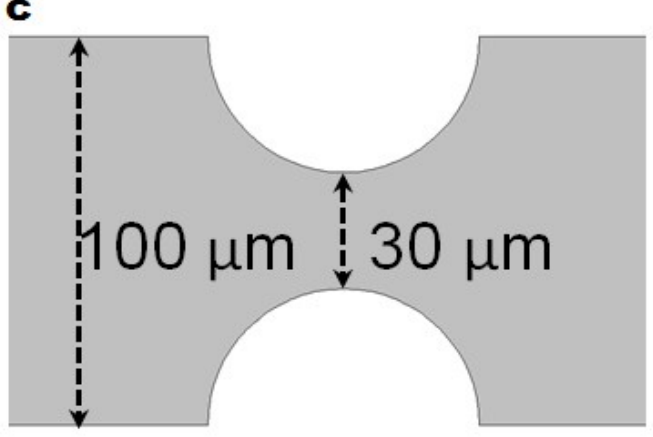

b

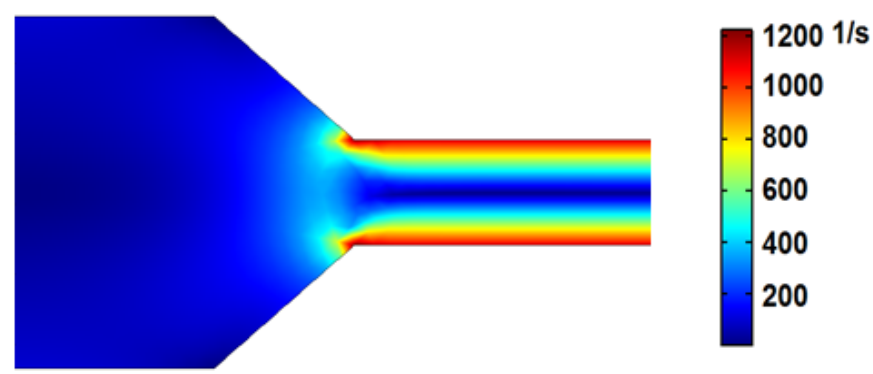

d

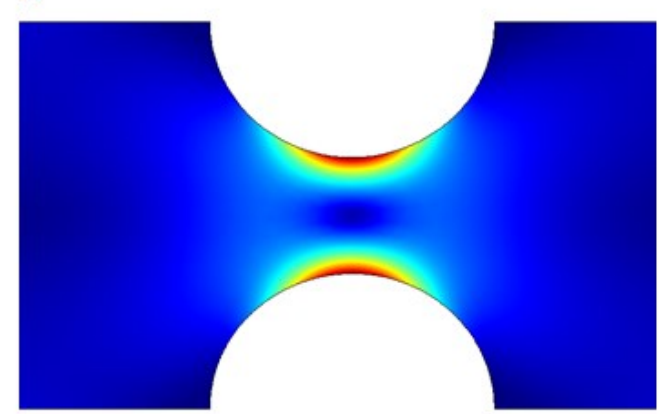

$12001 / \mathrm{s}$

1000

800

600

400

200

Figure 8.1 a) A microfluidic microchannel design to study the released ATP from erythrocytes under shear flow. The confined region was designed to apply sudden change of shear stress on the perfused red blood cells. b) The shear rate distribution under the flow of $0.3 \mu \mathrm{L} / \mathrm{min}$. c) a microvessel model was designed to mimic a clotting microvessel. d) The corresponding shear rate under the flow of $0.3 \mu \mathrm{L} / \mathrm{min}$. 


\section{Reference}

Abate, A. R., D. Lee, et al. (2008). "Glass coating for PDMS microfluidic channels by sol-gel methods." Lab Chip 8(4): 516-518.

Abdelgawad, M., C. Wu, et al. (2011). "A fast and simple method to fabricate circular microchannels in polydimethylsiloxane (PDMS)." Lab on a Chip 11(3): 545-551.

Abgrall, P. and A. Gue (2007). "Lab-on-chip technologies: making a microfluidic network and coupling it into a complete microsystem - a review." Journal of Micromechanics and Microengineering 17(5): R15.

Adeosun, J. T. and A. Lawal (2009). "Numerical and experimental studies of mixing characteristics in a $\mathrm{T}$-junction microchannel using residence-time distribution." Chemical Engineering Science 64(10): 2422-2432.

Agarwal, A., N. Ranganathan, et al. (2008). "Self-sealed circular channels for micro-fluidics." Sensors and Actuators A: Physical 142(1): 80-87.

Aitkenhead, M., S.-J. Wang, et al. (2002). "Identification of Endothelial Cell Genes Expressed in an in Vitro Model of Angiogenesis: Induction of ESM-1, ßigh3, and NrCAM." Microvascular research 63(2): 159-171.

Andriopoulou, P., P. Navarro, et al. (1999). "Histamine induces tyrosine phosphorylation of endothelial cell-to-cell adherens junctions." Arterioscler Thromb Vasc Biol 19(10): 2286-2297.

Azzi, S., J. K. Hebda, et al. (2013). "Vascular permeability and drug delivery in cancers." Front Oncol 3: 211.

Bazant, M. Z. and T. M. Squires (2004). "Induced-charge electrokinetic phenomena: theory and microfluidic applications." Physical Review Letters 92(6): 066101.

Becker, H. and C. Gärtner (2008). "Polymer microfabrication technologies for microfluidic systems." Analytical and bioanalytical chemistry 390(1): 89111.

Bellan, L. M., S. P. Singh, et al. (2009). "Fabrication of an artificial 3-dimensional vascular network using sacrificial sugar structures." Soft Matter 5(7): 13541357.

Bernardo, A., C. Ball, et al. (2004). "Effects of inflammatory cytokines on the release and cleavage of the endothelial cell-derived ultralarge von Willebrand factor multimers under flow." Blood 104(1): 100-106.

Bernatchez, P., A. Sharma, et al. (2011). "A noninhibitory mutant of the caveolin-1 scaffolding domain enhances eNOS-derived NO synthesis and vasodilation in mice." Journal of Clinical Investigation 121(9): 3747-3755. 
Berthier, E. and D. J. Beebe (2007). "Flow rate analysis of a surface tension driven passive micropump." Lab Chip 7(11): 1475-1478.

Berthier, E., E. W. Young, et al. (2012). "Engineers are from PDMS-land, Biologists are from Polystyrenia." Lab on a Chip 12(7): 1224-1237.

Bhatia, S. N. and D. E. Ingber (2014). "Microfluidic organs-on-chips." Nat Biotechnol 32(8): 760-772.

Bischel, L. L., E. W. Young, et al. (2013). "Tubeless microfluidic angiogenesis assay with three-dimensional endothelial-lined microvessels." Biomaterials 34(5): 1471-1477.

Bolender, R. P. (1974). "Stereological analysis of the guinea pig pancreas. I. Analytical model and quantitative description of nonstimulated pancreatic exocrine cells." J Cell Biol 61(2): 269-287.

Bonini, M. G., R. O. Dull, et al. (2014). "Caveolin-1 Regulation of Endothelial Nitric Oxide Synthase (eNOS) Function and Oxidative Stress in the Endothelium." Systems Biology of Free Radicals and Antioxidants: 13431363.

Booth, R. and H. Kim (2012). "Characterization of a microfluidic in vitro model of the blood-brain barrier ( $\mu$ BBB)." Lab on a Chip 12(10): 1784-1792.

Borenstein, J. T., M. M. Tupper, et al. (2010). "Functional endothelialized microvascular networks with circular cross-sections in a tissue culture substrate." Biomedical microdevices 12(1): 71-79.

Bouiis, D., G. A. Hospers, et al. (2001). "Endothelium in vitro: a review of human vascular endothelial cell lines for blood vessel-related research." Angiogenesis 4(2): 91-102.

Boy, D. A., F. Gibou, et al. (2008). "Simulation tools for lab on a chip research: advantages, challenges, and thoughts for the future." Lab Chip 8(9): 14241431.

Brantley-Sieders, D. M., W. B. Fang, et al. (2006). "Ephrin-A1 facilitates mammary tumor metastasis through an angiogenesis-dependent mechanism mediated by EphA receptor and vascular endothelial growth factor in mice." Cancer research 66(21): 10315-10324.

Bredt, D. S., P. M. Hwang, et al. (1991). "Cloned and expressed nitric oxide synthase structurally resembles cytochrome P-450 reductase." Nature 351(6329): 714-718.

Breslin, J. W., P. J. Pappas, et al. (2003). "VEGF increases endothelial permeability by separate signaling pathways involving ERK-1/2 and nitric oxide." Am J Physiol Heart Circ Physiol 284(1): H92-H100.

Brown, R. C. and T. P. Davis (2002). "Calcium Modulation of Adherens and Tight Junction Function A Potential Mechanism for Blood-Brain Barrier Disruption After Stroke." Stroke 33(6): 1706-1711. 
Bugyi, B. and M. F. Carlier (2010). "Control of actin filament treadmilling in cell motility." Annu Rev Biophys 39: 449-470.

BurakáOzdoganlar, O. (2011). "Fabrication of circular microfluidic channels by combining mechanical micromilling and soft lithography." Lab on a Chip 11(8): 1550-1555.

Burgazli, M. K., N. Aydogdu, et al. (2013). "Effects of caffeic acid phenethyl ester (CAPE) on membrane potential and intracellular calcium in human endothelial cells." European review for medical and pharmacological sciences 17(6): 720-728.

Burnstock, G. and C. Kennedy (1986). "A dual function for adenosine 5'triphosphate in the regulation of vascular tone. Excitatory cotransmitter with noradrenaline from perivascular nerves and locally released inhibitory intravascular agent." Circ Res 58(3): 319-330.

Byun, C. K., K. Abi-Samra, et al. (2014). "Pumps for microfluidic cell culture." Electrophoresis 35(2-3): 245-257.

Camp, J. P., T. Stokol, et al. (2008). "Fabrication of a multiple-diameter branched network of microvascular channels with semi-circular cross-sections using xenon difluoride etching." Biomedical microdevices 10(2): 179-186.

Cao, J., S. Usami, et al. (1997). "Development of a side-view chamber for studying cell-surface adhesion under flow conditions." Annals of biomedical engineering 25(3): 573-580.

Capani, F., M. E. Martone, et al. (2001). "Selective localization of high concentrations of F-actin in subpopulations of dendritic spines in rat central nervous system: a three-dimensional electron microscopic study." J Comp Neurol 435(2): 156-170.

Carafoli, E. (1987). "Intracellular calcium homeostasis." Annual review of biochemistry 56(1): 395-433.

Carmeliet, P. and R. K. Jain (2000). "Angiogenesis in cancer and other diseases." Nature 407(6801): 249-257.

Carmignoto, G., L. Pasti, et al. (1999). "Intracellular calcium pools and calcium oscillations in cells from the central nervous system." Calcium as a Cellular Regulator: 55 .

Carter, T. D., T. J. Hallam, et al. (1988). "Regulation of P2y-purinoceptormediated prostacyclin release from human endothelial cells by cytoplasmic calcium concentration." Br J Pharmacol 95(4): 1181-1190.

Cha, W., Y.-C. Tung, et al. (2010). "Patterned electrode-based amperometric gas sensor for direct nitric oxide detection within microfluidic devices." Analytical chemistry 82(8): 3300-3305.

Chandra, A., S. Barillas, et al. (2007). "A novel fluorescence-based cellular permeability assay." J Biochem Biophys Methods 70(3): 329-333. 
Chau, L., M. Doran, et al. (2009). "A novel multishear microdevice for studying cell mechanics." Lab on a Chip 9(13): 1897-1902.

Chen, J., P. R. Somanath, et al. (2005). "Akt1 regulates pathological angiogenesis, vascular maturation and permeability in vivo." Nat Med 11(11): 1188-1196.

Chen, L., E. E. Tredget, et al. (2008). "Paracrine factors of mesenchymal stem cells recruit macrophages and endothelial lineage cells and enhance wound healing." PloS one 3(4): e1886.

Chen, Y.-C., G.-Y. Chen, et al. (2010). "A lab-on-a-chip capillary network for red blood cell hydrodynamics." Microfluidics and Nanofluidics 9(2-3): 585-591.

Chien, S., S. Li, et al. (1998). "Effects of mechanical forces on signal transduction and gene expression in endothelial cells." Hypertension 31(1): 162-169.

Chin, D. and A. R. Means (2000). "Calmodulin: a prototypical calcium sensor." Trends Cell Biol 10(8): 322-328.

Chin, L., J. Yu, et al. (2011). "Production of reactive oxygen species in endothelial cells under different pulsatile shear stresses and glucose concentrations." Lab on a Chip 11(11): 1856-1863.

Chiu, J.-J. and S. Chien (2011). "Effects of disturbed flow on vascular endothelium: pathophysiological basis and clinical perspectives." Physiological reviews 91(1): 327-387.

Cho, B. S., T. G. Schuster, et al. (2003). "Passively driven integrated microfluidic system for separation of motile sperm." Anal Chem 75(7): 1671-1675.

Chrobak, K. M., D. R. Potter, et al. (2006). "Formation of perfused, functional microvascular tubes in vitro." Microvascular research 71(3): 185-196.

Chung, S., R. Sudo, et al. (2009). "Cell migration into scaffolds under co-culture conditions in a microfluidic platform." Lab on a Chip 9(2): 269-275.

Clough, G., C. C. Michel, et al. (1988). "Inflammatory changes in permeability and ultrastructure of single vessels in the frog mesenteric microcirculation." $\underline{\mathrm{J}}$ Physiol 395: 99-114.

Colgan, O. C., G. Ferguson, et al. (2007). "Regulation of bovine brain microvascular endothelial tight junction assembly and barrier function by laminar shear stress." American Journal of Physiology-Heart and Circulatory Physiology 292(6): H3190-H3197.

Collier, T. O., J. M. Anderson, et al. (2004). "Inhibition of macrophage development and foreign body giant cell formation by hydrophilic interpenetrating polymer network." Journal of Biomedical Materials Research Part A 69(4): 644-650.

Conway, D. E., M. R. Williams, et al. (2010). "Endothelial cell responses to atheroprone flow are driven by two separate flow components: low timeaverage shear stress and fluid flow reversal." American Journal of Physiology-Heart and Circulatory Physiology 298(2): H367-H374. 
Corada, M., M. Mariotti, et al. (1999). "Vascular endothelial-cadherin is an important determinant of microvascular integrity in vivo." Proc Natl Acad Sci U S A 96(17): 9815-9820.

Corson, M. A., N. L. James, et al. (1996). "Phosphorylation of endothelial nitric oxide synthase in response to fluid shear stress." Circ Res 79(5): 984-991.

Couzon, C., A. Duperray, et al. (2009). "Critical stresses for cancer cell detachment in microchannels." European Biophysics Journal 38(8): 1035-1047.

Craig, A. G., G. E. Grau, et al. (2012). "The role of animal models for research on severe malaria." PLoS pathogens 8(2): e1002401.

Crespi, A., Y. Gu, et al. (2010). "Three-dimensional Mach-Zehnder interferometer in a microfluidic chip for spatially-resolved label-free detection." Lab on a Chip 10(9): 1167-1173.

Cucullo, L., P.-O. Couraud, et al. (2007). "Immortalized human brain endothelial cells and flow-based vascular modeling: a marriage of convenience for rational neurovascular studies." Journal of Cerebral Blood Flow \& Metabolism 28(2): 312-328.

Curry, F. E. (1992). "Modulation of venular microvessel permeability by calcium influx into endothelial cells." FASEB J 6(7): 2456-2466.

Daly, D., R. F. Stevens, et al. (1990). "The Manufacture of Microlenses by Melting Photoresist." Measurement Science \& Technology 1(8): 759-766.

Darhuber, A. A. and S. M. Troian (2005). "Principles of microfluidic actuation by modulation of surface stresses." Annu. Rev. Fluid Mech. 37: 425-455.

Davies, P. F. (2008). "Hemodynamic shear stress and the endothelium in cardiovascular pathophysiology." Nature Clinical Practice Cardiovascular Medicine 6(1): 16-26.

Davies, P. F., T. Mundel, et al. (1995). "A mechanism for heterogeneous endothelial responses to flow in vivo and in vitro." J Biomech 28(12): 15531560 .

Davis, G. E., K. J. Bayless, et al. (2002). "Molecular basis of endothelial cell morphogenesis in three - dimensional extracellular matrices." The Anatomical Record 268(3): 252-275.

de Boer, M. J., R. W. Tjerkstra, et al. (2000). "Micromachining of buried micro channels in silicon." Microelectromechanical Systems, Journal of 9(1): 94103.

De Gennes, P.-G. (1985). "Wetting: statics and dynamics." Reviews of modern physics 57(3): 827.

Dejana, E. (2004). "Endothelial cell-cell junctions: happy together." Nat Rev Mol Cell Biol 5(4): 261-270. 
Dejana, E., F. Orsenigo, et al. (2008). "The role of adherens junctions and VEcadherin in the control of vascular permeability." J Cell Sci 121(Pt 13): 2115-2122.

Dejana, E., E. Tournier-Lasserve, et al. (2009). "The control of vascular integrity by endothelial cell junctions: molecular basis and pathological implications." Developmental cell 16(2): 209-221.

Dekker, R. J., S. van Soest, et al. (2002). "Prolonged fluid shear stress induces a distinct set of endothelial cell genes, most specifically lung Krüppel-like factor (KLF2)." Blood 100(5): 1689-1698.

Dietrich, H. H., T. Horiuchi, et al. (2009). "Mechanism of ATP-induced local and conducted vasomotor responses in isolated rat cerebral penetrating arterioles." J Vasc Res 46(3): 253-264.

Dimmeler, S., I. Fleming, et al. (1999). "Activation of nitric oxide synthase in endothelial cells by Akt-dependent phosphorylation." Nature 399(6736): 601-605.

Dixon, J. B. (2006). A biomedical engineering approach to investigating flow and wall shear stress in contracting lymphatics, Doctoral dissertation, Texas A\&M University.

Dolan, J. M., H. Meng, et al. (2011). "High fluid shear stress and spatial shear stress gradients affect endothelial proliferation, survival, and alignment." Ann Biomed Eng 39(6): 1620-1631.

Donovan, D., N. Brown, et al. (2001). "Comparison of three in vitro human 'angiogenesis' assays with capillaries formed in vivo." Angiogenesis 4(2): 113-121.

Douville, N. J., Y.-C. Tung, et al. (2010). "Fabrication of two-layered channel system with embedded electrodes to measure resistance across epithelial and endothelial barriers." Analytical chemistry 82(6): 2505-2511.

Dredge, K., R. Horsfall, et al. (2005). "Orally administered lenalidomide (CC-5013) is anti-angiogenic in vivo and inhibits endothelial cell migration and Akt phosphorylation in vitro." Microvascular research 69(1): 56-63.

Duansak, N. and G. W. Schmid-Schönbein (2013). "The oxygen free radicals control MMP-9 and transcription factors expression in the spontaneously hypertensive rat." Microvascular research 90: 154-161.

Dubyak, G. R. and C. el-Moatassim (1993). "Signal transduction via P2-purinergic receptors for extracellular ATP and other nucleotides." Am J Physiol 265(3 Pt 1): C577-606.

Dudek, S. M. and J. G. Garcia (2001). "Cytoskeletal regulation of pulmonary vascular permeability." J Appl Physiol (1985) 91(4): 1487-1500. 
Duffy, D. C., J. C. McDonald, et al. (1998). "Rapid prototyping of microfluidic systems in poly (dimethylsiloxane)." Analytical chemistry 70(23): 49744984.

El-Ali, J., P. K. Sorger, et al. (2006). "Cells on chips." Nature 442(7101): 403-411. ElectronicMaterials, A. (2003, 4/03/2003). "AZ® P4000 Thick Film Photoresist Datasheet." Retrieved 8/19, 2014, from http://www.clean.cise.columbia.edu/process/p4000vfr1.pdf.

Elias, H.-G. (1997). An introduction to polymer science, VCH.

Emerson, D. R., K. Cieslicki, et al. (2006). "Biomimetic design of microfluidic manifolds based on a generalised Murray's law." Lab Chip 6(3): 447-454.

Félétou, M. (2011). Calcium signaling in vascular cells and cell-to-cell communications. The Endothelium: Part 1: Multiple Functions of the Endothelial Cells-Focus on Endothelium-Derived Vasoactive Mediators. San Rafael (CA), Morgan \& Claypool Life Sciences.

Félétou, M. (2011). Multiple Functions of the Endothelial Cells. The Endothelium: Part 1: Multiple Functions of the Endothelial Cells-Focus on EndotheliumDerived Vasoactive Mediators. San Rafael (CA), Morgan \& Claypool Life Sciences.

Feletou, M. and P. M. Vanhoutte (2006). "Endothelial dysfunction: a multifaceted disorder (The Wiggers Award Lecture)." Am J Physiol Heart Circ Physiol 291(3): H985-1002.

Feng, Z., S. Fukuda, et al. (2011). "Flux characteristics of cell culture medium in rectangular microchannels." Journal of Artificial Organs 14(3): 238-244.

Feugier, P., R. Black, et al. (2005). "Attachment, morphology and adherence of human endothelial cells to vascular prosthesis materials under the action of shear stress." Biomaterials 26(13): 1457-1466.

Fiddes, L. K., N. Raz, et al. (2010). "A circular cross-section PDMS microfluidics system for replication of cardiovascular flow conditions." Biomaterials 31(13): 3459-3464.

Fisher, A. B., S. Chien, et al. (2001). "Endothelial cellular response to altered shear stress." Am J Physiol Lung Cell Mol Physiol 281(3): L529-533.

Fleming, I. and R. Busse (1999). "Signal transduction of eNOS activation." Cardiovasc Res 43(3): 532-541.

Fleming, I. and R. Busse (2003). "Molecular mechanisms involved in the regulation of the endothelial nitric oxide synthase." Am J Physiol Regul Integr Comp Physiol 284(1): R1-12.

Folkman, J. (1972). "Angiogenesis in psoriasis: therapeutic implications." J Invest Dermatol 59(1): 40-43. 
Franke, H., H.-J. Galla, et al. (2000). "Primary cultures of brain microvessel endothelial cells: a valid and flexible model to study drug transport through the blood-brain barrier in vitro." Brain Research Protocols 5(3): 248-256.

Franke, R.-P., M. Gräfe, et al. (1984). "Induction of human vascular endothelial stress fibres by fluid shear stress."

Fukumura, D. (2005). Window Models. Microvascular Research: Biology and Pathology. D. Shepro. Burlington, Elsevier Academic Press. 1: 151-157.

Gafiychuk, V. V. and I. A. Lubashevsky (2001). "On the principles of the vascular network branching." J Theor Biol 212(1): 1-9.

Galbraith, C., R. Skalak, et al. (1998). "Shear stress induces spatial reorganization of the endothelial cell cytoskeleton." Cell motility and the cytoskeleton 40(4): 317-330.

Ge, Y., T. Deng, et al. (2009). "Dynamic monitoring of changes in endothelial cellsubstrate adhesiveness during leukocyte adhesion by microelectrical impedance assay." Acta biochimica et biophysica Sinica 41(3): 256-262.

Gee, K. R., K. A. Brown, et al. (2000). "Chemical and physiological characterization of fluo-4 $\mathrm{Ca}(2+)$-indicator dyes." Cell Calcium 27(2): 97106.

Gellersen, B., K. Reimann, et al. (2010). "Invasiveness of human endometrial stromal cells is promoted by decidualization and by trophoblast-derived signals." Hum Reprod 25(4): 862-873.

Glatzel, T., C. Litterst, et al. (2008). "Computational fluid dynamics (CFD) software tools for microfluidic applications-A case study." Computers \& Fluids 37(3): 218-235.

Golden, A. P. and J. Tien (2007). "Fabrication of microfluidic hydrogels using molded gelatin as a sacrificial element." Lab Chip 7(6): 720-725.

Griep, L., F. Wolbers, et al. (2013). "BBB ON CHIP: microfluidic platform to mechanically and biochemically modulate blood-brain barrier function." Biomedical microdevices 15(1): 145-150.

Grunwald, D. J. and J. S. Eisen (2002). "Headwaters of the zebrafish -- emergence of a new model vertebrate." Nat Rev Genet 3(9): 717-724.

Gumbiner, B. M. (2005). "Regulation of cadherin-mediated adhesion in morphogenesis." Nat Rev Mol Cell Biol 6(8): 622-634.

Hatch, A., G. Hansmann, et al. (2011). "Engineered alginate hydrogels for effective microfluidic capture and release of endothelial progenitor cells from whole blood." Langmuir 27(7): 4257-4264.

He, P. and F. E. Curry (1991). "Depolarization modulates endothelial cell calcium influx and microvessel permeability." Am J Physiol 261(4 Pt 2): H12461254. 
He, P. and F. E. Curry (1993). "Differential actions of cAMP on endothelial $[\mathrm{Ca} 2+] \mathrm{i}$ and permeability in microvessels exposed to ATP." Am J Physiol 265(3 Pt 2): H1019-1023.

He, P. and F. E. Curry (1994). "Endothelial cell hyperpolarization increases [Ca2+]i and venular microvessel permeability." J Appl Physiol (1985) 76(6): 2288-2297.

He, P., S. N. Pagakis, et al. (1990). "Measurement of cytoplasmic calcium in single microvessels with increased permeability." Am J Physiol 258(5 Pt 2): H1366-1374.

He, P., X. Zhang, et al. (1996). "Ca2+ entry through conductive pathway modulates receptor-mediated increase in microvessel permeability." American Journal of Physiology-Heart and Circulatory Physiology 271(6): H2377-H2387.

He, P., X. Zhang, et al. (1996). "Ca2+ entry through conductive pathway modulates receptor-mediated increase in microvessel permeability." Am J Physiol 271(6 Pt 2): H2377-2387.

Heino, J. (2007). "The collagen family members as cell adhesion proteins." Bioessays 29(10): 1001-1010.

Holton, M., T. M. Mohamed, et al. (2010). "Endothelial nitric oxide synthase activity is inhibited by the plasma membrane calcium ATPase in human endothelial cells." Cardiovasc Res 87(3): 440-448.

$\mathrm{Hu}, \mathrm{G}$. and D. Li (2007). "Multiscale phenomena in microfluidics and nanofluidics." Chemical Engineering Science 62(13): 3443-3454.

Huang, S. B., S. S. Wang, et al. (2013). "An integrated microfluidic cell culture system for high-throughput perfusion three-dimensional cell culture-based assays: effect of cell culture model on the results of chemosensitivity assays." Lab Chip 13(6): 1133-1143.

Huang, Z., X. Li, et al. (2012). "Microfabrication of cylindrical microfluidic channel networks for microvascular research." Biomedical microdevices 14(5): 873-883.

Hudry-Clergeon, H., D. Stengel, et al. (2005). "Platelet-activating factor increases VE-cadherin tyrosine phosphorylation in mouse endothelial cells and its association with the PtdIns3'-kinase." FASEB J 19(6): 512-520.

Huh, D., G. A. Hamilton, et al. (2011). "From 3D cell culture to organs-on-chips." Trends Cell Biol 21(12): 745-754.

Hulvey, M. K. and R. S. Martin (2009). "A microchip-based endothelium mimic utilizing open reservoirs for cell immobilization and integrated carbon ink microelectrodes for detection." Analytical and bioanalytical chemistry 393(2): 599-605. 
HwanáSung, J. (2011). "Microscale 3-D hydrogel scaffold for biomimetic gastrointestinal (GI) tract model." Lab on a Chip 11(3): 389-392.

Ibrahim, M. and M. K Richardson (2013). "Microfluidic Devices for Cell, Tissue and Embryo Culture." Recent Patents on Regenerative Medicine 3(3): 249263.

Ingber, D. E. (2002). "Mechanical signaling and the cellular response to extracellular matrix in angiogenesis and cardiovascular physiology." $\underline{\text { Circ }}$ Res 91(10): 877-887.

Jain, R. K., L. L. Munn, et al. (2011). Transparent window models and intravital microscopy: imaging gene expression, physiological function and therapeutic effects in tumors. Tumor models in cancer research, Springer: 641-679.

Jay, T. R. and M. B. Stern (1994). "Preshaping Photoresist for Refractive Microlens Fabrication." Optical Engineering 33(11): 3552-3555.

Jeong, J.-Y., H.-B. Kwon, et al. (2008). "Functional and developmental analysis of the blood-brain barrier in zebrafish." Brain research bulletin 75(5): 619-628.

Jho, D., D. Mehta, et al. (2005). "Angiopoietin-1 opposes VEGF-induced increase in endothelial permeability by inhibiting TRPC1-dependent $\mathrm{Ca} 2$ influx." Circulation research 96(12): 1282-1290.

Jiang, S., R. Cheng, et al. (2013). "Real-time electrical detection of nitric oxide in biological systems with sub-nanomolar sensitivity." Nature communications 4.

Jo, H., R. Dull, et al. (1991). "Endothelial albumin permeability is shear dependent, time dependent, and reversible." American Journal of Physiology-Heart and Circulatory Physiology 260(6): H1992-H1996.

Kadohama, T., N. Akasaka, et al. (2006). "p38 Mitogen-activated protein kinase activation in endothelial cell is implicated in cell alignment and elongation induced by fluid shear stress." Endothelium 13(1): 43-50.

Kaihara, S., J. Borenstein, et al. (2000). "Silicon micromachining to tissue engineer branched vascular channels for liver fabrication." Tissue Eng 6(2): 105-117.

Kang, Y. B., S. Rawat, et al. (2013). "Layered long-term co-culture of hepatocytes and endothelial cells on a transwell membrane: toward engineering the liver sinusoid." Biofabrication 5(4): 045008.

Kannan, R. Y., H. J. Salacinski, et al. (2005). "The roles of tissue engineering and vascularisation in the development of micro-vascular networks: a review." Biomaterials 26(14): 1857-1875.

Kantak, A. S., B. K. Gale, et al. (2003). "Platelet function analyzer: Shear activation of platelets in microchannels." Biomedical microdevices 5(3): 207-215. 
Kanzawa, S., H. Endo, et al. (1993). "Improved in vitro angiogenesis model by collagen density reduction and the use of type III collagen." Annals of plastic surgery 30(3): 244-251.

Karpanen, T. and S. Schulte-Merker (2011). "Zebrafish provides a novel model for lymphatic vascular research." Methods Cell Biol 105: 223-238.

Kelly-Goss, M. R., R. S. Sweat, et al. (2013). "Vascular islands during microvascular regression and regrowth in adult networks." Frontiers in physiology 4.

Kessel, R. G. and R. H. Kardon (1979). Tissues and organs: a text-atlas of scanning electron microscopy, WH Freeman San Francisco.

Kim, S., H. Lee, et al. (2013). "Engineering of functional, perfusable 3D microvascular networks on a chip." Lab Chip 13(8): 1489-1500.

Kiosses, W. B., S. J. Shattil, et al. (2001). "Rac recruits high-affinity integrin $\alpha v \beta 3$ to lamellipodia in endothelial cell migration." Nature cell biology 3(3): 316320.

Kirby, B. J. (2010). Micro-and nanoscale fluid mechanics: transport in microfluidic devices, Cambridge University Press.

Kobayashi, H., M. Ogawa, et al. (2010). "New strategies for fluorescent probe design in medical diagnostic imaging." Chem Rev 110(5): 2620-2640.

Kojima, H., N. Nakatsubo, et al. (1998). "Detection and imaging of nitric oxide with novel fluorescent indicators: diaminofluoresceins." Anal Chem 70(13): 2446-2453.

Kojima, H., N. Nakatsubo, et al. (1998). "Detection and imaging of nitric oxide with novel fluorescent indicators: diaminofluoresceins." Analytical chemistry 70(13): 2446-2453.

Kojima, H., Y. Urano, et al. (1999). "Fluorescent indicators for imaging nitric oxide production." Angewandte Chemie International Edition 38(21): 32093212.

Kotsis, D. H. and D. M. Spence (2003). "Detection of ATP-induced nitric oxide in a biomimetic circulatory vessel containing an immobilized endothelium." Analytical chemistry 75(1): 145-151.

Kou, S., L. Pan, et al. (2011). "A multishear microfluidic device for quantitative analysis of calcium dynamics in osteoblasts." Biochem Biophys Res Commun 408(2): 350-355.

$\mathrm{Ku}, \mathrm{S}$. H., J. Ryu, et al. (2010). "General functionalization route for cell adhesion on non-wetting surfaces." Biomaterials 31(9): 2535-2541.

Lai, A. K. and A. C. Lo (2013). "Animal models of diabetic retinopathy: summary and comparison." J Diabetes Res 2013: 106594. 
Lal, B. K., S. Varma, et al. (2001). "VEGF increases permeability of the endothelial cell monolayer by activation of $\mathrm{PKB} / \mathrm{akt}$, endothelial nitric-oxide synthase, and MAP kinase pathways." Microvasc Res 62(3): 252-262.

LaMack, J. A. and M. H. Friedman (2007). "Individual and combined effects of shear stress magnitude and spatial gradient on endothelial cell gene expression." Am J Physiol Heart Circ Physiol 293(5): H2853-2859.

Lampugnani, M. G. and E. Dejana (1997). "Interendothelial junctions: structure, signalling and functional roles." Current opinion in cell biology 9(5): 674682.

Lampugnani, M. G. and E. Dejana (2007). "Adherens junctions in endothelial cells regulate vessel maintenance and angiogenesis." Thrombosis research 120: S1-S6.

Lancaster, J. R. and B. Gaston (2004). "NO and nitrosothiols: spatial confinement and free diffusion." American Journal of Physiology-Lung Cellular and Molecular Physiology 287(3): L465-L466.

Lane, W. O., A. E. Jantzen, et al. (2012). "Parallel-plate flow chamber and continuous flow circuit to evaluate endothelial progenitor cells under laminar flow shear stress." Journal of visualized experiments: JoVE(59).

Langille, B. L., J. J. Graham, et al. (1991). "Dynamics of shear-induced redistribution of F-actin in endothelial cells in vivo." Arterioscler Thromb 11(6): 1814-1820.

Laschke, M. W., B. Vollmar, et al. (2011). "The Dorsal Skinfold Chamber: Window into the Dynamic Interaction of Biomaterials with Their Surrounding Host Tissue." European Cells \& Materials 22: 147-167.

Lee, C. M., L. J. Robinson, et al. (1995). "Oligomerization of endothelial nitric oxide synthase. Evidence for a dominant negative effect of truncation mutants." J Biol Chem 270(46): 27403-27406.

Lee, E. J. and L. E. Niklason (2010). "A novel flow bioreactor for in vitro microvascularization." Tissue Engineering Part C: Methods 16(5): 11911200.

Lee, E. J., G. Vunjak-Novakovic, et al. (2009). "A biocompatible endothelial cell delivery system for in vitro tissue engineering." Cell transplantation 18(7): 731.

Lee, H., S. M. Dellatore, et al. (2007). "Mussel-inspired surface chemistry for multifunctional coatings." Science 318(5849): 426-430.

Lee, K. H., S. J. Shin, et al. (2009). "Synthesis of Cell - Laden Alginate Hollow Fibers Using Microfluidic Chips and Microvascularized Tissue Engineering Applications." Small 5(11): 1264-1268. 
Lee, M.-J., S. Thangada, et al. (1999). "Vascular endothelial cell adherens junction assembly and morphogenesis induced by sphingosine-1-phosphate." Cell 99(3): 301-312.

Lehle, K., R. H. Straub, et al. (2010). "Relevance of disease - and organ - specific endothelial cells for in vitro research." Cell biology international 34(12): 1231-1238.

Leong, T. G., A. M. Zarafshar, et al. (2010). "Three - Dimensional Fabrication at Small Size Scales." Small 6(7): 792-806.

Letourneau, S., L. Hernandez, et al. (2010). "Evaluating the effects of estradiol on endothelial nitric oxide stimulated by erythrocyte-derived ATP using a microfluidic approach." Anal Bioanal Chem 397(8): 3369-3375.

Letourneau, S., L. Hernandez, et al. (2010). "Evaluating the effects of estradiol on endothelial nitric oxide stimulated by erythrocyte-derived ATP using a microfluidic approach." Analytical and bioanalytical chemistry 397(8): 3369-3375.

Li, Y.-S. J., J. H. Haga, et al. (2005). "Molecular basis of the effects of shear stress on vascular endothelial cells." Journal of biomechanics 38(10): 1949-1971.

Lim, D., Y. Kamotani, et al. (2003). "Fabrication of microfluidic mixers and artificial vasculatures using a high-brightness diode-pumped Nd : YAG laser direct write method." Lab on a Chip 3(4): 318-323.

Lipowsky, H. H. (1995). Shear stress in the circulation. Flow-Dependent Regulation of Vascular Function, Springer: 28-45.

Little, C., V. Mironov, et al. (1998). Vascular morphogenesis: in vivo, in vitro, in mente, Springer.

Liu, R. H., M. A. Stremler, et al. (2000). "Passive mixing in a three-dimensional serpentine microchannel." Microelectromechanical Systems, Journal of 9(2): 190-197.

Lu, H., L. Y. Koo, et al. (2004). "Microfluidic shear devices for quantitative analysis of cell adhesion." Anal Chem 76(18): 5257-5264.

Luckhoff, A. and R. Busse (1990). "Calcium influx into endothelial cells and formation of endothelium-derived relaxing factor is controlled by the membrane potential." Pflugers Arch 416(3): 305-311.

Lukacs, N. W., R. Strieter, et al. (1995). "Production of chemokines, interleukin-8 and monocyte chemoattractant protein-1, during monocyte: endothelial cell interactions." Blood 86(7): 2767-2773.

Malek, A. M., R. Ahlquist, et al. (1995). "A cone-plate apparatus for the in vitro biochemical and molecular analysis of the effect of shear stress on adherent cells." Methods in cell science 17(3): 165-176. 
Malek, A. M., S. L. Alper, et al. (1999). "Hemodynamic shear stress and its role in atherosclerosis." Jama 282(21): 2035-2042.

Malek, A. M. and S. Izumo (1996). "Mechanism of endothelial cell shape change and cytoskeletal remodeling in response to fluid shear stress." J Cell Sci $\mathbf{1 0 9}$ ( Pt 4): 713-726.

Mannix, R. J., T. Moatter, et al. (1993). "Cellular signaling responses mediated by a novel nucleotide receptor in rabbit microvessel endothelium." $\underline{\text { Am J }}$ Physiol 265(2 Pt 2): H675-680.

Mao, X., L. Xie, et al. (2014). "Flow-induced regulation of brain endothelial cells in vitro." Vascul Pharmacol 62(2): 82-87.

Marchenko, S. M. and S. O. Sage (1993). "Electrical properties of resting and acetylcholine-stimulated endothelium in intact rat aorta." J Physiol 462: 735751.

Mariampillai, A., B. A. Standish, et al. (2008). "Speckle variance detection of microvasculature using swept-source optical coherence tomography." Opt Lett 33(13): 1530-1532.

Marsden, P. A., K. T. Schappert, et al. (1992). "Molecular cloning and characterization of human endothelial nitric oxide synthase." $\underline{\text { FEBS Lett }}$ 307(3): 287-293.

Maselli, V., R. Osellame, et al. (2006). "Fabrication of long microchannels with circular cross section using astigmatically shaped femtosecond laser pulses and chemical etching." Applied physics letters 88(19): 191107.

McCann, J. A., S. D. Peterson, et al. (2005). "Non-uniform flow behavior in a parallel plate flow chamber : alters endothelial cell responses." Ann Biomed Eng 33(3): 328-336.

McCullough, W. T., D. M. Collins, et al. (1997). "Arteriolar responses to extracellular ATP in striated muscle." Am J Physiol 272(4 Pt 2): H18861891.

McDonald, D. M. (1994). "Endothelial gaps and permeability of venules in rat tracheas exposed to inflammatory stimuli." American Journal of PhysiologyLung Cellular and Molecular Physiology 10(1): L61.

McDonald, J. C. and G. M. Whitesides (2002). "Poly (dimethylsiloxane) as a material for fabricating microfluidic devices." Accounts of chemical research 35(7): 491-499.

McDonald, J. C. and G. M. Whitesides (2002). "Poly(dimethylsiloxane) as a material for fabricating microfluidic devices." Accounts of chemical research 35(7): 491-499.

Meeson, A., M. Palmer, et al. (1996). "A relationship between apoptosis and flow during programmed capillary regression is revealed by vital analysis." Development 122(12): 3929-3938. 
Mehling, M. and S. Tay (2014). "Microfluidic cell culture." Curr Opin Biotechnol 25: $95-102$.

Mehta, D. and A. B. Malik (2006). "Signaling mechanisms regulating endothelial permeability." Physiol Rev 86(1): 279-367.

Melchior, B. and J. A. Frangos (2012). "Gaq/11-mediated/11-mediated intracellular calcium responses to retrograde flow in endothelial cells." Am J Physiol Cell Physiol 303(4): C467-473.

Meyvantsson, I., J. W. Warrick, et al. (2008). "Automated cell culture in high density tubeless microfluidic device arrays." Lab Chip 8(5): 717-724.

Michel, C. C. and F. E. Curry (1999). "Microvascular permeability." Physiol Rev 79(3): 703-761.

Michelot, A. and D. G. Drubin (2011). "Building distinct actin filament networks in a common cytoplasm." Curr Biol 21(14): R560-569.

Miles, A. and E. M. Miles (1952). "Vascular reactions to histamine, histamineliberator and leukotaxine in the skin of guinea-pigs." The Journal of physiology 118(2): 228-257.

Millán, J., R. J. Cain, et al. (2010). "Adherens junctions connect stress fibres between adjacent endothelial cells." BMC biology 8(1): 11.

Morawietz, H., R. Talanow, et al. (2000). "Regulation of the endothelin system by shear stress in human endothelial cells." The Journal of physiology 525(3): 761-770.

Morgan, J. R. and M. L. Yarmush (1999). Tissue engineering methods and protocols, Springer.

Muller, W. A. (2003). "Leukocyte-endothelial-cell interactions in leukocyte transmigration and the inflammatory response." Trends in immunology 24(6): 326-333.

Munaron, L. (2006). "Intracellular calcium, endothelial cells and angiogenesis." Recent patents on anti-cancer drug discovery 1(1): 105-119.

Murohara, T., J. R. Horowitz, et al. (1998). "Vascular endothelial growth factor/vascular permeability factor enhances vascular permeability via nitric oxide and prostacyclin." Circulation 97(1): 99-107.

Murray, C. D. (1926). "The Physiological Principle of Minimum Work Applied to the Angle of Branching of Arteries." J Gen Physiol 9(6): 835-841.

Murray, C. D. (1926). "The physiological principle of minimum work: I. The vascular system and the cost of blood volume." Proceedings of the National Academy of Sciences of the United States of America 12(3): 207.

Nagano, T. (2010). "Development of fluorescent probes for bioimaging applications." Proc Jpn Acad Ser B Phys Biol Sci 86(8): 837-847. 
Nagel, T., N. Resnick, et al. (1994). "Shear stress selectively upregulates intercellular adhesion molecule-1 expression in cultured human vascular endothelial cells." Journal of Clinical Investigation 94(2): 885.

Nakatsu, M. N., R. C. Sainson, et al. (2003). "Angiogenic sprouting and capillary lumen formation modeled by human umbilical vein endothelial cells (HUVEC) in fibrin gels: the role of fibroblasts and Angiopoietin-1 2 ." Microvascular research 66(2): 102-112.

Navarro-Antolín, J., M. J. López-Muñoz, et al. (2001). "Formation of peroxynitrite in vascular endothelial cells exposed to cyclosporine A." The FASEB journal 15(7): 1291-1293.

Nerem, R. M., R. W. Alexander, et al. (1998). "The study of the influence of flow on vascular endothelial biology." The American journal of the medical sciences 316(3): 169-175.

Nerem, R. M., R. W. Alexander, et al. (1998). "The study of the influence of flow on vascular endothelial biology." Am J Med Sci 316(3): 169-175.

Nguyen, D.-H. T., S. C. Stapleton, et al. (2013). "Biomimetic model to reconstitute angiogenic sprouting morphogenesis in vitro." Proceedings of the National Academy of Sciences 110(17): 6712-6717.

Nichol, J. W., S. T. Koshy, et al. (2010). "Cell-laden microengineered gelatin methacrylate hydrogels." Biomaterials 31(21): 5536-5544.

Nicoli, S., D. Ribatti, et al. (2007). "Mammalian tumor xenografts induce neovascularization in zebrafish embryos." Cancer Res 67(7): 2927-2931.

Nobe, K., T. Sone, et al. (2005). "Thrombin-induced force development in vascular endothelial cells: contribution to alteration of permeability mediated by calcium-dependent and -independent pathways." J Pharmacol Sci 99(3): 252-263.

Noria, S., D. B. Cowan, et al. (1999). "Transient and steady-state effects of shear stress on endothelial cell adherens junctions." Circulation research 85(6): 504-514.

Norrby, K. (1998). "Microvascular density in terms of number and length of microvessel segments per unit tissue volume in mammalian angiogenesis." Microvascular research 55(1): 43-53.

Norrby, K. C. (2011). "Rat mesentery angiogenesis assay." Journal of visualized experiments: JoVE(52).

O'Brien, S., N. J. Kent, et al. (2012). "Effective hydrodynamic shaping of sample streams in a microfluidic parallel-plate flow-assay device: matching whole blood dynamic viscosity." Biomedical Engineering, IEEE Transactions on 59(2): 374-382.

O'Neill, F. T. and J. T. Sheridan (2002). "Photoresist reflow method of microlens production Part I: Background and experiments." Optik 113(9): 391-404. 
O'Neill, F. T. and J. T. Sheridan (2002). "Photoresist reflow method of microlens production Part I: Background and experiments." Optik-International Journal for Light and Electron Optics 113(9): 391-404.

Oblak, T. D., P. Root, et al. (2006). "Fluorescence monitoring of ATP-stimulated, endothelium-derived nitric oxide production in channels of a poly(dimethylsiloxane)-based microfluidic device." Analytical chemistry 78(9): 3193-3197.

Orsenigo, F., C. Giampietro, et al. (2012). "Phosphorylation of VE-cadherin is modulated by haemodynamic forces and contributes to the regulation of vascular permeability in vivo." Nat Commun 3: 1208.

Ota, H. and N. Miki (2011). "Microfluidic experimental platform for producing size-controlled three-dimensional spheroids." Sensors and Actuators aPhysical 169(2): 266-273.

Papenfuss, H. D., J. F. Gross, et al. (1979). "A transparent access chamber for the rat dorsal skin fold." Microvasc Res 18(3): 311-318.

Pardridge, W. M. (2006). Introduction to the blood-brain barrier: methodology, biology and pathology, Cambridge University Press.

Paredes, R. M., J. C. Etzler, et al. (2008). "Chemical calcium indicators." Methods 46(3): 143-151.

Park, J. Y., C. M. Hwang, et al. (2007). "Gradient generation by an osmotic pump and the behavior of human mesenchymal stem cells under the fetal bovine serum concentration gradient." Lab on a Chip 7(12): 1673-1680.

Park, J. Y., S. K. Kim, et al. (2009). "Differentiation of neural progenitor cells in a microfluidic chip-generated cytokine gradient." Stem Cells 27(11): 26462654.

Parsa, H., R. Upadhyay, et al. (2011). "Uncovering the behaviors of individual cells within a multicellular microvascular community." Proceedings of the National Academy of Sciences 108(12): 5133-5138.

Pawley, J. (2010). Handbook of biological confocal microscopy, Springer.

Peled, A., V. Grabovsky, et al. (1999). "The chemokine SDF-1 stimulates integrinmediated arrest of CD34+ cells on vascular endothelium under shear flow." Journal of Clinical Investigation 104(9): 1199-1211.

Pi, L., X. Ding, et al. (2008). "Connective tissue growth factor with a novel fibronectin binding site promotes cell adhesion and migration during rat oval cell activation." Hepatology 47(3): 996-1004.

Plouffe, B. D., M. Radisic, et al. (2008). "Microfluidic depletion of endothelial cells, smooth muscle cells, and fibroblasts from heterogeneous suspensions." Lab Chip 8(3): 462-472. 
Poinsot, T. J., amp, et al. (1992). "Boundary conditions for direct simulations of compressible viscous flows." Journal of computational physics 101(1): 104129.

Pollard, T. D. and J. A. Cooper (2009). "Actin, a central player in cell shape and movement." Science 326(5957): 1208-1212.

Pollard, T. D. and J. A. Cooper (2009). "Actin, a central player in cell shape and movement." Science 326(5957): 1208-1212.

Potter, C. M., M. H. Lundberg, et al. (2011). "Role of shear stress in endothelial cell morphology and expression of cyclooxygenase isoforms." Arteriosclerosis, thrombosis, and vascular biology 31(2): 384-391.

Price, G. M., K. H. Wong, et al. (2010). "Effect of mechanical factors on the function of engineered human blood microvessels in microfluidic collagen gels." Biomaterials 31(24): 6182-6189.

Qin, K. R., W. Y. Jiang, et al. (1998). "On analysis of the steady flow in an irrectangular parallel-plate flow chamber." Applied Mathematics and Mechanics-English Edition 19(9): 851-859.

Rabiet, M.-J., J.-L. Plantier, et al. (1996). "Thrombin-induced increase in endothelial permeability is associated with changes in cell-to-cell junction organization." Arteriosclerosis, thrombosis, and vascular biology 16(3): 488496.

Rabiet, M. J., J. L. Plantier, et al. (1996). "Thrombin-induced increase in endothelial permeability is associated with changes in cell-to-cell junction organization." Arterioscler Thromb Vasc Biol 16(3): 488-496.

Ranjan, V., Z. Xiao, et al. (1995). "Constitutive NOS expression in cultured endothelial cells is elevated by fluid shear stress." American Journal of Physiology-Heart and Circulatory Physiology 38(2): H550.

Raynaud, C. M., J. M. Butler, et al. (2013). "Endothelial cells provide a niche for placental hematopoietic stem/progenitor cell expansion through broad transcriptomic modification." Stem cell research 11(3): 1074-1090.

Renkin, E. M., F. E. Curry, et al. (1974). "Failure of histamine, 5hydroxytryptamine, or bradykinin to increase capillary permeability to plasma proteins in frogs: action of compound 48/80." Microvasc Res 8(2): 213-217.

Resnick, N., H. Yahav, et al. (2003). "Fluid shear stress and the vascular endothelium: for better and for worse." Progress in biophysics and molecular biology 81(3): 177-199.

Reymond, N., B. B. d'Água, et al. (2013). "Crossing the endothelial barrier during metastasis." Nature Reviews Cancer 13(12): 858-870. 
Ribatti, D., B. Nico, et al. (2001). "Chorioallantoic membrane capillary bed: A useful target for studying angiogenesis and anti - angiogenesis in vivo." The Anatomical Record 264(4): 317-324.

Richards, T. A. and T. Cavalier-Smith (2005). "Myosin domain evolution and the primary divergence of eukaryotes." Nature 436(7054): 1113-1118.

Riha, G. M., P. H. Lin, et al. (2005). "Roles of hemodynamic forces in vascular cell differentiation." Annals of biomedical engineering 33(6): 772-779.

Rivero-Vilches, F. J., S. de Frutos, et al. (2003). "Differential relaxing responses to particulate or soluble guanylyl cyclase activation on endothelial cells: a mechanism dependent on PKG-I alpha activation by NO/cGMP." Am J Physiol Cell Physiol 285(4): C891-898.

Rodriguez, I., P. Spicar-Mihalic, et al. (2003). "Rapid prototyping of glass microchannels." Analytica Chimica Acta 496(1): 205-215.

Rogers, J. A. and R. G. Nuzzo (2005). "Recent progress in soft lithography." Materials today 8(2): 50-56.

Rousseau, S., F. Houle, et al. (1997). "p38 MAP kinase activation by vascular endothelial growth factor mediates actin reorganization and cell migration in human endothelial cells." Oncogene 15(18): 2169-2177.

Sackmann, E. K., A. L. Fulton, et al. (2014). "The present and future role of microfluidics in biomedical research." Nature 507(7491): 181-189.

Saleh, F., M. Whyte, et al. (2011). "Effects of endothelial cells on human mesenchymal stem cell activity in a three-dimensional in vitro model." Eur Cell Mater 22: 242-257.

Sato, M. and T. Ohashi (2005). "Biorheological views of endothelial cell responses to mechanical stimuli." Biorheology 42(6): 421-441.

Schaper, W. (2001). "Therapeutic arteriogenesis has arrived." Circulation 104(17): 1994-1995.

Seebach, J., P. Dieterich, et al. (2000). "Endothelial barrier function under laminar fluid shear stress." Laboratory investigation 80(12): 1819-1831.

Sekimoto, K., R. Oguma, et al. (1987). "Morphological Stability Analysis of Partial Wetting." Annals of Physics 176(2): 359-392.

Senger, D. R., C. A. Perruzzi, et al. (2002). "The $\alpha 1 \beta 1$ and $\alpha 2 \beta 1$ integrins provide critical support for vascular endothelial growth factor signaling, endothelial cell migration, and tumor angiogenesis." The American journal of pathology 160(1): 195.

Seo, C.-T., C.-H. Bae, et al. (2004). "Fabrication of circular-type microchannel using photoresist reflow and isotropic etching for microfluidic devices." Japanese journal of applied physics 43(11R): 7773.

Shamloo, A., N. Ma, et al. (2008). "Endothelial cell polarization and chemotaxis in a microfluidic device." Lab Chip 8(8): 1292-1299. 
Shao, J., L. Wu, et al. (2009). "Integrated microfluidic chip for endothelial cells culture and analysis exposed to a pulsatile and oscillatory shear stress." Lab on a Chip 9(21): 3118-3125.

Shao, J., L. Wu, et al. (2010). "A microfluidic chip for permeability assays of endothelial monolayer." Biomedical microdevices 12(1): 81-88.

Shay-Salit, A., M. Shushy, et al. (2002). "VEGF receptor 2 and the adherens junction as a mechanical transducer in vascular endothelial cells." Proceedings of the National Academy of Sciences of the United States of America 99(14): 9462-9467.

Shen, Q., S. K. Goderie, et al. (2004). "Endothelial cells stimulate self-renewal and expand neurogenesis of neural stem cells." Science 304(5675): 1338-1340.

Shepro, D. (2005). Microvascular Research: Biology and Pathology, Elsevier.

Sherman, T. F. (1981). "On connecting large vessels to small. The meaning of Murray's law." The Journal of general physiology 78(4): 431-453.

Shevkoplyas, S. S., S. C. Gifford, et al. (2003). "Prototype of an in vitro model of the microcirculation." Microvasc Res 65(2): 132-136.

Shin, Y., S. Han, et al. (2012). "Microfluidic assay for simultaneous culture of multiple cell types on surfaces or within hydrogels." Nature protocols 7(7): 1247-1259.

Shiu, Y.-T., S. Li, et al. (2004). "Rho mediates the shear-enhancement of endothelial cell migration and traction force generation." Biophysical journal 86(4): 2558-2565.

Shyy, Y. J., H. J. Hsieh, et al. (1994). "Fluid shear stress induces a biphasic response of human monocyte chemotactic protein 1 gene expression in vascular endothelium." Proc Natl Acad Sci U S A 91(11): 4678-4682.

Song, J. W., S. P. Cavnar, et al. (2009). "Microfluidic endothelium for studying the intravascular adhesion of metastatic breast cancer cells." PloS one 4(6): e5756.

Song, S.-H., C.-K. Lee, et al. (2010). "A rapid and simple fabrication method for 3dimensional circular microfluidic channel using metal wire removal process." Microfluidics and Nanofluidics 9(2-3): 533-540.

Sonoda, S., C. Spee, et al. (2009). "A protocol for the culture and differentiation of highly polarized human retinal pigment epithelial cells." Nature protocols 4(5): 662-673.

Spence, D. M., N. J. Torrence, et al. (2004). "Amperometric determination of nitric oxide derived from pulmonary artery endothelial cells immobilized in a microchip channel." Analyst 129(11): 995-1000.

Spindel, O. N., R. M. Burke, et al. (2014). "Thioredoxin-interacting protein is a biomechanical regulator of Src activity: key role in endothelial cell stress fiber formation." Circ Res 114(7): 1125-1132. 
Sprague, R. S., J. J. Olearczyk, et al. (2003). "Extracellular ATP signaling in the rabbit lung: erythrocytes as determinants of vascular resistance." $\underline{\text { Am J }}$ Physiol Heart Circ Physiol 285(2): H693-700.

Spring, K., C. Chabot, et al. (2012). "Tyrosine phosphorylation of DEP-1/CD148 as a mechanism controlling Src kinase activation, endothelial cell permeability, invasion, and capillary formation." Blood 120(13): 2745-2756.

Stalder, A. F., Z. Liu, et al. (2011). "Patient Specific Hemodynamics: Combined 4D Flow-Sensitive MRI and CFD." Computational Biomechanics for Medicine: Soft Tissues and the Musculoskeletal System: 27-38.

Stankevicius, E., V. Lopez-Valverde, et al. (2006). "Combination of Ca2+ activated $\mathrm{K}+$ channel blockers inhibits acetylcholine-evoked nitric oxide release in rat superior mesenteric artery." Br J Pharmacol 149(5): 560-572.

Steinberg, M. S. and P. M. McNutt (1999). "Cadherins and their connections: adhesion junctions have broader functions." Current opinion in cell biology 11(5): 554-560.

Stephenson, K. A., S. R. Banerjee, et al. (2004). "Bridging the gap between in vitro and in vivo imaging: isostructural Re and $99 \mathrm{mTc}$ complexes for correlating fluorescence and radioimaging studies." Journal of the American Chemical Society 126(28): 8598-8599.

Stokes, K. Y., D. Cooper, et al. (2002). "Hypercholesterolemia promotes inflammation and microvascular dysfunction: role of nitric oxide and superoxide." Free Radic Biol Med 33(8): 1026-1036.

Stone, H. A., A. D. Stroock, et al. (2004). "Engineering flows in small devices: microfluidics toward a lab-on-a-chip." Annu. Rev. Fluid Mech. 36: 381-411.

Stübs, C., O. Picker, et al. (2013). "Acute, short-term hypercapnia improves microvascular oxygenation of the colon in an animal model of sepsis." Microvascular research 90: 180-186.

$\mathrm{Su}, \mathrm{X}$., A. B. Theberge, et al. (2013). "Effect of microculture on cell metabolism and biochemistry: do cells get stressed in microchannels?" Analytical chemistry 85(3): 1562-1570.

Sucosky, P., M. Padala, et al. (2008). "Design of an ex vivo culture system to investigate the effects of shear stress on cardiovascular tissue." Journal of biomechanical engineering 130(3): 035001.

Sukardi, H., H. T. Chng, et al. (2011). "Zebrafish for drug toxicity screening: bridging the in vitro cell-based models and in vivo mammalian models." Expert opinion on drug metabolism \& toxicology 7(5): 579-589.

Takayama, S., J. C. McDonald, et al. (1999). "Patterning cells and their environments using multiple laminar fluid flows in capillary networks." Proceedings of the National Academy of Sciences 96(10): 5545-5548. 
Takayama, S., E. Ostuni, et al. (2001). "Laminar flows - Subcellular positioning of small molecules." Nature 411(6841): 1016-1016.

Takeshita, Y. and R. M. Ransohoff (2012). "Inflammatory cell trafficking across the blood-brain barrier: chemokine regulation and in vitro models." Immunological reviews 248(1): 228-239.

Tamilarasan, K. P., G. K. Kolluru, et al. (2006). "Thalidomide attenuates nitric oxide mediated angiogenesis by blocking migration of endothelial cells." BMC Cell Biol 7: 17.

Tang, D. G. and C. J. Conti (2004). Endothelial cell development, vasculogenesis, angiogenesis, and tumor neovascularization: an update. Seminars in thrombosis and hemostasis, Copyright(C) 2004 by Thieme Medical Publishers, Inc., 333 Seventh Avenue, New York, NY 10001, USA.

Tang, M. D., A. P. Golden, et al. (2004). "Fabrication of Collagen Gels That Contain Patterned, Micrometer - Scale Cavities." Advanced materials 16(15): 1345-1348.

Tarbell, J. M. (2010). "Shear stress and the endothelial transport barrier." Cardiovascular research 87(2): 320-330.

Thayer, S. A. and R. J. Miller (1990). "Regulation of the intracellular free calcium concentration in single rat dorsal root ganglion neurones in vitro." The Journal of physiology 425(1): 85-115.

Thomas, D. D., X. Liu, et al. (2001). "The biological lifetime of nitric oxide: implications for the perivascular dynamics of NO and O2." Proceedings of the National Academy of Sciences 98(1): 355-360.

Tojkander, S., G. Gateva, et al. (2012). "Actin stress fibers-assembly, dynamics and biological roles." Journal of cell science 125(8): 1855-1864.

Tong, J. Z., P. De Lagausie, et al. (1992). "Long-term culture of adult rat hepatocyte spheroids." Exp Cell Res 200(2): 326-332.

Tran, L., J. Farinas, et al. (2005). "Agonist-induced calcium response in single human platelets assayed in a microfluidic device." Analytical biochemistry 341(2): 361-368.

Tsai, M., A. Kita, et al. (2012). "In vitro modeling of the microvascular occlusion and thrombosis that occur in hematologic diseases using microfluidic technology." J Clin Invest 122(1): 408-418.

Tsai, M., A. Kita, et al. (2012). "In vitro modeling of the microvascular occlusion and thrombosis that occur in hematologic diseases using microfluidic technology." Journal of Clinical Investigation 122(1): 408-418.

Tzima, E., M. A. del Pozo, et al. (2001). "Activation of integrins in endothelial cells by fluid shear stress mediates Rho - dependent cytoskeletal alignment." The EMBO journal 20(17): 4639-4647. 
Tzima, E., M. Irani-Tehrani, et al. (2005). "A mechanosensory complex that mediates the endothelial cell response to fluid shear stress." Nature 437(7057): 426-431.

Tzima, E., M. Irani-Tehrani, et al. (2005). "A mechanosensory complex that mediates the endothelial cell response to fluid shear stress." Nature 437(7057): 426-431.

Tzima, E., J. Reader, et al. (2003). "Biologically active fragment of a human tRNA synthetase inhibits fluid shear stress-activated responses of endothelial cells." Proceedings of the National Academy of Sciences 100(25): 1490314907.

Ukropec, J. A., M. K. Hollinger, et al. (2002). "Regulation of VE-cadherin linkage to the cytoskeleton in endothelial cells exposed to fluid shear stress." Experimental cell research 273(2): 240-247.

Van der Meer, A., A. Poot, et al. (2010). "Analyzing shear stress-induced alignment of actin filaments in endothelial cells with a microfluidic assay." Biomicrofluidics 4(1): 011103.

van der Meer, A. D., A. A. Poot, et al. (2009). "Microfluidic technology in vascular research." J Biomed Biotechnol 2009: 823148.

Van Kooten, T., J. Schakenraad, et al. (1992). "Development and use of a parallel - plate flow chamber for studying cellular adhesion to solid surfaces." Journal of biomedical materials research 26(6): 725-738.

van Nieuw Amerongen, G. P., R. Draijer, et al. (1998). "Transient and Prolonged Increase in Endothelial permeability induced by histamine and thrombin role of protein kinases, calcium, and RhoA." Circulation research 83(11): 11151123.

van Royen, N., J. J. Piek, et al. (2001). "Arteriogenesis: mechanisms and modulation of collateral artery development." Journal of Nuclear Cardiology 8(6): 687-693.

Velazquez, O. C., R. Snyder, et al. (2002). "Fibroblast-dependent differentiation of human microvascular endothelial cells into capillary-like 3-dimensional networks." The FASEB journal 16(10): 1316-1318.

Vilahur, G., T. Padro, et al. (2011). "Atherosclerosis and thrombosis: insights from large animal models." BioMed research international 2011.

Voinov, O. (1999). "Dynamic edge angles of wetting upon spreading of a drop over a solid surface." Journal of applied mechanics and technical physics 40(1): 86-92.

Walker, G. M., H. C. Zeringue, et al. (2004). "Microenvironment design considerations for cellular scale studies." Lab Chip 4(2): 91-97. 
Walsh, T. G., R. P. Murphy, et al. (2011). "Stabilization of brain microvascular endothelial barrier function by shear stress involves VE - cadherin signaling leading to modulation of pTyr - occludin levels." Journal of cellular physiology 226(11): 3053-3063.

Wan, J., W. D. Ristenpart, et al. (2008). "Dynamics of shear-induced ATP release from red blood cells." Proc Natl Acad Sci U S A 105(43): 16432-16437.

Wang, G.-J., K.-H. Ho, et al. (2007). "Microvessel scaffold with circular microchannels by photoresist melting." Biomedical microdevices 9(5): 657663.

Warabi, E., Y. Wada, et al. (2004). "Effect on endothelial cell gene expression of shear stress, oxygen concentration, and low-density lipoprotein as studied by a novel flow cell culture system." Free Radical Biology and Medicine 37(5): 682-694.

Wei, Y. C. and B. D. Bai (2013). "The Exploitation and Simulation of a Microfluidic Device Research on the Response of Cell Fluid Shear Stress." Advanced Materials Research 647: 357-362.

Weis, S. M. and D. A. Cheresh (2005). "Pathophysiological consequences of VEGF-induced vascular permeability." Nature 437(7058): 497-504.

Weis, W. I. and W. J. Nelson (2006). "Re-solving the cadherin-catenin-actin conundrum." J Biol Chem 281(47): 35593-35597.

Wheeler, A. R., W. R. Throndset, et al. (2003). "Microfluidic device for single-cell analysis." Analytical chemistry 75(14): 3581-3586.

Whitesides, G. M. (2006). "The origins and the future of microfluidics." Nature 442(7101): 368-373.

Whitesides, G. M., E. Ostuni, et al. (2001). "Soft lithography in biology and biochemistry." Annual review of biomedical engineering 3(1): 335-373.

Wilting, J., B. Christ, et al. (1993). "In vivo effects of vascular endothelial growth factor on the chicken chorioallantoic membrane." Cell and tissue research 274(1): 163-172.

Wójciak-Stothard, B., L. Williams, et al. (1999). "Monocyte adhesion and spreading on human endothelial cells is dependent on Rho-regulated receptor clustering." The Journal of cell biology 145(6): 1293-1307.

Wysolmerski, R. B. and D. Lagunoff (1990). "Involvement of myosin light-chain kinase in endothelial cell retraction." Proc Natl Acad Sci U S A 87(1): 16-20.

Xia, Y., M. Prawirasatya, et al. (2011). "Seeding density matters: extensive intercellular contact masks the surface dependence of endothelial cellbiomaterial interactions." Journal of materials science: Materials in medicine 22(2): 389-396. 
Xia, Y. and G. M. Whitesides (1998). "Soft lithography." Annual review of materials science 28(1): 153-184.

Xu, S., X. Zhou, et al. (2013). "Caveolin-1 scaffolding domain promotes leukocyte adhesion by reduced basal endothelial nitric oxide-mediated ICAM-1 phosphorylation in rat mesenteric venules." American Journal of Physiology-Heart and Circulatory Physiology 305(10): H1484-H1493.

$\mathrm{Xu}, \mathrm{S}$., X. Zhou, et al. (2013). "Caveolin-1 scaffolding domain promotes leukocyte adhesion by reduced basal endothelial nitric oxide-mediated ICAM-1 phosphorylation in rat mesenteric venules." Am J Physiol Heart Circ Physiol 305(10): H1484-1493.

$\mathrm{Xu}, \mathrm{T}$., C.-W. Li, et al. (2010). "Microfluidic formation of single cell array for parallel analysis of $\mathrm{Ca}<\sup >2+</$ sup $>$ release-activated $\mathrm{Ca}<\sup >$ $2+</$ sup $>$ (CRAC) channel activation and inhibition." Analytical biochemistry 396(2): 173-179.

$\mathrm{Xu}$, T., W. Yue, et al. (2013). "Microfluidics study of intracellular calcium response to mechanical stimulation on single suspension cells." Lab Chip 13(6): 1060-1069.

Yamamoto, K., K. Furuya, et al. (2011). "Visualization of flow-induced ATP release and triggering of $\mathrm{Ca} 2+$ waves at caveolae in vascular endothelial cells." J Cell Sci 124(Pt 20): 3477-3483.

Yegutkin, G. G. (2008). "Nucleotide- and nucleoside-converting ectoenzymes: Important modulators of purinergic signalling cascade." Biochim Biophys Acta 1783(5): 673-694.

Yeow, Y. L., Y. K. Leong, et al. (2008). "Slow steady viscous flow of newtonian fluids in parallel-disk viscometer with wall slip." Journal of Applied Mechanics-Transactions of the Asme 75(4).

Yi, F. X., A. Y. Zhang, et al. (2002). "Simultaneous in situ monitoring of intracellular $\mathrm{Ca} 2+$ and $\mathrm{NO}$ in endothelium of coronary arteries." $\underline{\mathrm{Am} \mathrm{J}}$ Physiol Heart Circ Physiol 283(6): H2725-2732.

Yin, H., X. Zhang, et al. (2007). "Influence of hydrodynamic conditions on quantitative cellular assays in microfluidic systems." Analytical chemistry 79(18): 7139-7144.

Young, B., P. Woodford, et al. (2013). Wheater's functional histology: a text and colour atlas, Elsevier Health Sciences.

Young, E. W., M. W. Watson, et al. (2010). "Technique for real-time measurements of endothelial permeability in a microfluidic membrane chip using laser-induced fluorescence detection." Analytical chemistry 82(3): 808-816. 
Young, E. W., A. R. Wheeler, et al. (2007). "Matrix-dependent adhesion of vascular and valvular endothelial cells in microfluidic channels." Lab Chip 7(12): 1759-1766.

Yuan, D. and P. He (2012). "Vascular remodeling alters adhesion protein and cytoskeleton reactions to inflammatory stimuli resulting in enhanced permeability increases in rat venules." Journal of Applied Physiology 113(7): 1110-1120.

Yuan, D., S. Xu, et al. (2014). "Enhanced permeability responses to inflammation in streptozotocin-induced diabetic rat venules: Rho-mediated alterations of actin cytoskeleton and VE-cadherin." Am J Physiol Heart Circ Physiol 307(1): H44-53.

Yuan, S. Y. and R. R. Rigor (2010). Methods for Measuring Permeability. Regulation of Endothelial Barrier Function. S. Y. Yuan and R. R. Rigor. San Rafael (CA), Morgan \& Claypool Life Sciences.

Yuan, S. Y. and R. R. Rigor (2010). "Regulation of endothelial barrier function."

Yuan, S. Y. and R. R. Rigor (2010). "Structure and Function of Exchange Microvessels."

Zadeh, M. H., C. A. Glass, et al. (2008). "VEGF - Mediated Elevated Intracellular Calcium and Angiogenesis in Human Microvascular Endothelial Cells In Vitro are Inhibited by Dominant Negative TRPC6." Microcirculation 15(7): 605-614.

Zamir, M. and J. A. Medeiros (1982). "Arterial branching in man and monkey." $\underline{\mathrm{J}}$ Gen Physiol 79(3): 353-360.

Zeng, Y., T. S. Lee, et al. (2006). "Mass transport and shear stress in a microchannel bioreactor: numerical simulation and dynamic similarity." $\underline{\mathrm{J}}$ Biomech Eng 128(2): 185-193.

Zeng, Y. and J. M. Tarbell (2014). "The adaptive remodeling of endothelial glycocalyx in response to fluid shear stress." PloS one 9(1): e86249.

Zervantonakis, I. K., S. K. Hughes-Alford, et al. (2012). "Three-dimensional microfluidic model for tumor cell intravasation and endothelial barrier function." Proc Natl Acad Sci U S A 109(34): 13515-13520.

Zervantonakis, I. K., S. K. Hughes-Alford, et al. (2012). "Three-dimensional microfluidic model for tumor cell intravasation and endothelial barrier function." Proceedings of the National Academy of Sciences 109(34): $13515-13520$.

Zetter, B. R. (2008). "The scientific contributions of M. Judah Folkman to cancer research." Nature Reviews Cancer 8(8): 647-654.

Zhang, X., S. Kazerounian, et al. (2009). "Thrombospondin-1 modulates vascular endothelial growth factor activity at the receptor level." The FASEB journal 23(10): 3368-3376. 
Zheng, Y., J. Chen, et al. (2012). "In vitro microvessels for the study of angiogenesis and thrombosis." Proceedings of the National Academy of Sciences 109(24): 9342-9347.

Zheng, Y., J. Chen, et al. (2012). "In vitro microvessels for the study of angiogenesis and thrombosis." Proc Natl Acad Sci U S A 109(24): 93429347.

Zhou, X. and P. He (2010). "Endothelial [Ca2+]i and caveolin-1 antagonistically regulate eNOS activity and microvessel permeability in rat venules." Cardiovasc Res 87(2): 340-347.

Zhou, X. and P. He (2011). "Improved measurements of intracellular nitric oxide in intact microvessels using 4,5-diaminofluorescein diacetate." Am J Physiol Heart Circ Physiol 301(1): H108-114.

Zhou, X. and P. He (2011). "Temporal and spatial correlation of platelet-activating factor-induced increases in endothelial $[\mathrm{Ca} 2+] \mathrm{i}$, nitric oxide, and gap formation in intact venules." American Journal of Physiology-Heart and Circulatory Physiology 301(5): H1788-H1797.

Zhou, X., D. Yuan, et al. (2013). "H2O2-induced endothelial NO production contributes to vascular cell apoptosis and increased permeability in rat venules." American Journal of Physiology-Heart and Circulatory Physiology 304(1): H82-H93.

Zhu, L. and P. He (2005). "Platelet-activating factor increases endothelial $[\mathrm{Ca} 2+] \mathrm{i}$ and NO production in individually perfused intact microvessels." American Journal of Physiology-Heart and Circulatory Physiology 288(6): H2869$\mathrm{H} 2877$.

Zhu, L. and P. He (2005). "Platelet-activating factor increases endothelial $[\mathrm{Ca} 2+] \mathrm{i}$ and NO production in individually perfused intact microvessels." Am J Physiol Heart Circ Physiol 288(6): H2869-2877.

Zhu, X., L. Y. Chu, et al. (2004). "Arrays of horizontally-oriented mini-reservoirs generate steady microfluidic flows for continuous perfusion cell culture and gradient generation." Analyst 129(11): 1026-1031.

Zon, L. I. and R. T. Peterson (2005). "In vivo drug discovery in the zebrafish." Nat Rev Drug Discov 4(1): 35-44. 